\title{
On the Dynamical Stability and Instability of Parker Problem
}

\author{
Fei Jiang, ${ }^{\mathrm{a}, \mathrm{b}}$, Song Jiang ${ }^{\mathrm{c}}$ \\ ${ }^{a}$ College of Mathematics and Computer Science, Fuzhou University, Fuzhou 350108, China. \\ ${ }^{b}$ Key Laboratory of Operations Research and Control of Universities in Fujian, Fuzhou, 350108, China. \\ ${ }^{c}$ Institute of Applied Physics and Computational Mathematics, P.O. Box 8009, Beijing 100088, China.
}

\begin{abstract}
We investigate a Parker problem for the three-dimensional compressible isentropic viscous magnetohydrodynamic system with zero resistivity in the presence of a modified gravitational force in a vertical strip domain in which the velocity of the fluid is non-slip on the boundary, and focus on the stabilizing effect of the (equilibrium) magnetic field through the non-slip boundary condition. We show that there is a discriminant $\Xi$, depending on the known physical parameters, for the stability/instability of the Parker problem. More precisely, if $\Xi>0$, then the Parker problem is unstable, i.e., the Parker instability occurs, while if $\Xi<0$ and the initial perturbation satisfies some relations, then there exists a global (perturbation) solution which decays algebraically to zero in time, i.e., the Parker instability does not happen. The stability results in this paper reveal the stabilizing effect of the magnetic field through the non-slip boundary condition and the importance of boundary conditions upon the Parker instability, and demonstrate that a sufficiently strong magnetic field can prevent the Parker instability from occurring. In addition, based on the instability results, we further rigorously verify the Parker instability under Schwarzschild's or Tserkovnikov's instability conditions in the sense of Hadamard for a horizontally periodic domain.
\end{abstract}

Keywords: Compressible magnetohydrodynamic flow; Schwarzschild's criterion; Parker instability; magnetic buoyancy instability; stability.

\section{Introduction}

The equilibrium, in which a gas layer in a gravitational field is supported in part by a vertically decreasing horizontal magnetic field, is unstable [35], and such instability is called the Parker instability (or the magnetic buoyancy instability in some literatures). The behavior of the Parker instability can be described as follows. Suppose that magnetic field lines are disturbed and begin to undulate. The mass in the raised portion of a loop drains down along the field lines so that the loop to become lighter than the ambient medium. If the buoyancy at the loop top is larger than the restoring magnetics tension, the loop rises further and the instability sets in [57]. Parker noted that the falling mass accumulates in the magnetic valleys [51], and thus explained that some of the "large-scale" interstellar molecular cloud complexes can be formed in this way, since the growth rate of the instability is 10 times larger than that of the Jeans's gravitational instability of the gas cloud.

\footnotetext{
${ }^{*}$ Corresponding author.

Email addresses: jiangfei0591@163.com (Fei Jiang), jiang@iapcm.ac.cn (Song Jiang)
} 
Since Parker's pioneering work [51], many physicists have continued to develop the linear theory and nonlinear numerical simulation of the Parker instability, see [36, 37, 44, 45, 55] and the references cited therein. Moreover, the physicists have considered the Parker instability to be also one of the possible mechanisms for other various astrophysical phenomenon [20], such as, the rising and falling motions of gases above the spiral arm [59, 62], the molecular loops in the Galactic center [13], the Barnard loop in Orion, the rise and emergence of magnetic flux tubes in the Sun and other stars [2, 4, 46, 50] as well as in accretion disks [6], the jets ejected from the centers of active galaxies [52], and so on. Recently, Khalzov et.al. first used the Madison Plasma Couette Experiment to model the Parker instability [33].

It has been also widely investigated how the Parker instability evolves under the effects of other physical factors, such as rotation [36], cosmic rays [39, 41], corona [32], self-gravity [8], nonuniform gravitational fields [20], random magnetic fields [34], and so on. The effect of boundary conditions of the velocity on the evolution of the Parker instability was investigated in our previous article [27]. Based on the linearized motion equations, Jiang et.al. have found a new phenomenon that the non-slip velocity boundary condition, imposed on the direction of a (equilibrium) magnetic field, can enhance the stabilizing effect of the field, so that the Parker instability can be prevented under a sufficiently strong magnetic field. The main aims in this article are to rigorously prove this new phenomenon (i.e., the inhibition effect of a magnetic field on the nonlinear Parker instability through a non-slip boundary condition), and the criterion that gives the nonlinear Parker instability by developing new mathematical techniques based on the nonlinear motion equations. Before stating our results, we formulate the problem mathematically.

\subsection{Parker problem}

The verification of the stabilizing effect of a magnetic field on the Parker instability through a non-slip boundary condition and the investigation of the criteria leading to the occurrence of the Parker instability can be reduced to the proof of stability and instability for a Parker problem of the magnetohydrodynamic (MHD) equations, respectively. In this article, we consider the following three-dimensional (3D) compressible isentropic viscous MHD equations with zero resistivity (i.e., without magnetic diffusivity) in the presence of a gravitational field in a domain $\Omega \subset \mathbb{R}^{3}$ read as follows (see, for example, [9, 40] on the derivation of the motion equations).

$$
\left\{\begin{array}{l}
\rho_{t}+\operatorname{div}(\rho v)=0 \\
\rho v_{t}+\rho v \cdot \nabla v+\nabla\left(P+\lambda|M|^{2} / 2\right)=\mu_{1} \Delta v+\mu_{2} \nabla \operatorname{div} v+\lambda M \cdot \nabla M-\rho g e_{3} \\
M_{t}=M \cdot \nabla v-v \cdot \nabla M-M \operatorname{div} v \\
\operatorname{div} M=0
\end{array}\right.
$$

Here the unknowns $\rho:=\rho(x, t), v:=v(x, t)$ and $M:=M(x, t)$ denote the density, velocity and magnetic field of the compressible MHD fluid, respectively; $\lambda$ stands for the permeability of vacuum dividing by $4 \pi, g>0$ for the gravitational constant, $e_{3}=(0,0,1)^{\mathrm{T}}$ for the vertical unit vector, and $-\rho g e_{3}$ for the gravitational force. $\mu_{1}>0$ is the coefficient of shear viscosity and $\mu_{2}:=\nu+\mu_{1} / 3$ with $\nu$ being the positive bulk viscosity. The pressure $P$ is usually determined through the equations of state. In this article we focus our study on the case of polytropic gas:

$$
P \equiv P(\rho)=A \rho^{\gamma}
$$

where $\gamma \geq 1$ denotes the adiabatic index and $A>0$ is a constant. In the system (1.1) the equation $(1.1)_{1}$ is the continuity equation, $(1.1)_{2}$ describes the balance law of momentum, while (1.1) 3 is called the induction equation. As for the constraint $\operatorname{div} M=0$, it can be seen just as 
a restriction on the initial value of $M$ since $(\operatorname{div} M)_{t}=0$ due to $(1.1)_{3}$. We remark that the resistivity is neglected in $(1.1)_{3}$, and this arises in the physics regime with negligible electrical resistance.

Next, we construct a (magnetohydrostatic) equilibrium state $(\bar{\rho}, 0, \bar{M})$ to the MHD equations (1.1). Firstly, we choose a (equilibrium) density profile $\bar{\rho}:=\bar{\rho}\left(x_{3}\right) \in C^{1}(\bar{\Omega})$, which is independent of $\left(x_{1}, x_{2}\right)$ and satisfies

$$
\inf _{x \in \Omega} \bar{\rho}>0 .
$$

The condition (1.3) prevents us from treating vacuum. Then, for given $\bar{\rho}$ and $g$, we defined a horizontal magnetic field profile $\bar{M}:=m e_{1}:=(m, 0,0)^{T}$ with

$$
m \equiv m\left(x_{3}\right):= \pm \sqrt{\frac{2}{\lambda}(C-\bar{P}-F(g \bar{\rho}))}, \quad \text { a function of } x_{3} \text { only, }
$$

where $\bar{P}:=P(\bar{\rho})$ is the pressure profile, $F(g \bar{\rho})$ denotes a primitive function $g \bar{\rho}$ and $C$ is a positive constant satisfying

$$
\inf _{x \in \bar{\Omega}}\{C-\bar{P}-F(g \bar{\rho})\}>0 \text {. }
$$

It is easy to see that (1.4) makes sense for a bounded domain $\Omega$, and

$$
\bar{P}^{\prime}=-\lambda m m^{\prime}-g \bar{\rho}
$$

where $^{\prime}:=\mathrm{d} / \mathrm{d} x_{3}$. Moreover,

$$
\nabla\left(\bar{P}+\lambda|\bar{M}|^{2} / 2\right)=-g \bar{\rho} e_{3} \quad \text { and } \operatorname{div} \bar{M}=0,
$$

where $\lambda \bar{M} \cdot \nabla \bar{M}=0$. In what follows, we denote such equilibrium-state $(\bar{\rho}, 0, \bar{M})$ by $s_{e}$.

It is well-known that the equilibrium-state $s_{e}$ is unstable, if the density profile further satisfies Schwarzschild's (instability) condition 35]

$$
-\bar{\rho}^{\prime}\left(x_{3}^{0}\right)<\left.\frac{g \bar{\rho}^{2}}{\gamma \bar{P}}\right|_{x_{3}=x_{3}^{0}} \quad \text { for some } x_{3}^{0} \in\left\{x_{3} \mid\left(x_{1}, x_{2}, x_{3}\right)^{\mathrm{T}} \in \Omega\right\} .
$$

Such condition was not first found in the Parker instability problem, but in the (compressible thermal) convection problem studied by Schwarzschild in 1906 [56]. We mention that, for the convection problem, the pressure profile in Schwarzschild's condition not only depends on a density profile, but also on a temperature profile $\bar{T}$ due to the thermal effect, and often takes the form $\bar{P}=R \bar{\rho} \bar{T}$ with $R>0$ being a gas constant. Later, Tserkovnikov further investigated the convection problem in the presence of a horizontal magnetic field (abbreviated as the magnetic convection (MC) problem), and obtained an instability condition [63] in 1960:

$$
-g \bar{\rho}^{\prime}\left(x_{3}^{0}\right)<\left.\frac{g^{2} \bar{\rho}^{2}}{\gamma \bar{P}+\lambda m^{2}}\right|_{x_{3}=x_{3}^{0}} \quad \text { for some } x_{3}^{0} \in(-l, l) .
$$

However, in 1961, Newcomb [47] extended Tserkovnikov's analysis by imposing no constraints on the perturbation wave vectors and found that Schwarzschild's condition is not only the sufficient and necessary condition for the compressible thermal instability, but also for the instability in the MC problem, please refer to [68] for the physical interpretation for Newcomb's and Schwarzschild's conditions. 
After Parker's instability work in 1966, physicists further noted that the instability in the MC problem considered by Tserkovnikov and Newcomb not only involves the thermal instability, but also the Parker instability [35, 61, 64]]. In particular, for the isentropic case (i.e., one omits the thermal effect and the pressure is of form (1.2)), Schwarzschild's condition is equivalent to the magnetic buoyancy condition

$$
\left.\left(m^{2}\right)^{\prime}\right|_{x_{3}=x_{3}^{0}}<0 .
$$

by virtue of the relations (1.5) and (1.2). In other words, under Schwarzschild's condition, there exists a region, in which the strength of $\bar{M}$ is vertically decreasing. Thus the corresponding magnetic pressure in (1.6) plays a role of the magnetic buoyancy [50], which is able to support more mass against gravity than would be possible in its absence. In view of (1.7) and (1.9), we can see that the mechanism of the Parker instability refers to the pressure state and the magnetic buoyancy. To emphasis the mechanism of the magnetic buoyancy, the Parker instability is often called the magnetic buoyancy instability (or the ballooning instability in fusion plasma physics [58]).

Finally, we mention a special density profile, i.e., $\bar{\rho}$ satisfies the Rayleigh-Taylor condition

$$
\left.\bar{\rho}^{\prime}\right|_{x_{3}=x_{3}^{0}}>0
$$

for some $x_{3}^{0} \in\left\{x_{3} \mid\left(x_{1}, x_{2}, x_{3}\right) \in \Omega\right\}$. By (1.7), the equilibrium-state $s_{e}$ satisfying (1.10) is unstable. Since such density distribution is in close correspondence to the classical case of a heavy fluid supported by a light one, the Parker instability under such case is called the magnetic Rayleigh-Taylor instability [24], or the Kruskal-Schwarzschild instability due to the first investigation of Kruskal and Schwarzschild in 1953 [38], where they further pointed out that curvature of the magnetic lines can influence the development of instability.

Now, we introduce the Parker problem for the MHD equations around the equilibrium state $s_{e}$. Denoting the perturbation to the equilibrium state $s_{e}$ by

$$
\varrho=\rho-\bar{\rho}, \quad v=v-0, \quad N=M-\bar{M},
$$

and using the relations in (1.6), we obtain the perturbation equations:

$$
\left\{\begin{array}{l}
\varrho_{t}+\operatorname{div}((\varrho+\bar{\rho}) v)=0, \\
(\varrho+\bar{\rho}) v_{t}+(\varrho+\bar{\rho}) v \cdot \nabla v+\nabla\left(P(\varrho+\bar{\rho})+\lambda|N+\bar{M}|^{2} / 2\right) \\
\quad=\mu_{1} \Delta v+\mu_{2} \nabla \operatorname{div} v+\lambda(N+\bar{M}) \cdot \nabla(N+\bar{M})-(\varrho+\bar{\rho}) g e_{3}, \\
N_{t}=(N+\bar{M}) \cdot \nabla v-v \cdot \nabla(N+\bar{M})-(N+\bar{M}) \operatorname{div} v, \\
\operatorname{div} N=0 .
\end{array}\right.
$$

We impose the following initial and boundary conditions for (1.11):

$$
\begin{aligned}
& \left.(\varrho, v, N)\right|_{t=0}=\left(\varrho_{0}, v_{0}, N_{0}\right) \quad \text { in } \Omega, \\
& \left.v\right|_{\partial \Omega}=0 \quad \text { for any } t>0 .
\end{aligned}
$$

The linearized equations of (1.11) around the equilibrium state $s_{e}$ read as

$$
\left\{\begin{array}{l}
\varrho_{t}+\operatorname{div}(\bar{\rho} v)=0, \\
\bar{\rho} v_{t}+\nabla\left(P^{\prime}(\bar{\rho}) \varrho+\lambda m N_{1}\right)=\mu_{1} \Delta v+\mu_{2} \nabla \operatorname{div} v+\lambda \bar{M}^{\prime} N_{3}+\lambda m \partial_{1} N-\varrho g e_{3}, \\
N_{t}=m \partial_{1} v-v_{3} \bar{M}^{\prime}-\bar{M} \operatorname{div} v . \\
\operatorname{div} N=0,
\end{array}\right.
$$


where $P^{\prime}(\bar{\rho}):=\left.\left(A x_{3}^{\gamma}\right)^{\prime}\right|_{x_{3}=\bar{\rho}}$, and $N_{i}$ and $v_{i}$ denote the $i$-th component of $N$ and $v$, respectively. The system (1.14) with initial and boundary conditions (1.12)-(1.13) constitutes a linearized Parker problem, while the initial-boundary problem (1.11)-(1.13) is called the nonlinear Parker problem. The Parker instability, which is shown based on the linearized Parker problem, resp. the nonlinear Parker problem, is called the linear Parker instability, resp. the nonlinear Parker instability. At present, only the linear Parker instability under Schwarzschild's condition or Tserkovnikov's condition (1.8) is mathematically investigated, also for the case without viscosity.

\subsection{Criterion for stability/instability}

The linearized equations are convenient to analyze mathematically in order to have an insight into the physical and mathematical mechanisms of the Parker instability. Moreover, applying the energy principle [5] to the linearized Parker problem (1.12)-(1.14), Jiang et.al. have obtained criteria of stability/instability for the linearized Parker problem [27]. More precisely, in the case of a bounded domain $\Omega$, one has

(1) if $\Xi<0$, then the linearized Parker problem is stable;

(2) if $\Xi>0$, then the linearized Parker problem is unstable,

where

$$
\Xi:=\sup _{w \in H_{0}^{1}(\Omega)} \frac{E(w)}{\int_{\Omega} \lambda\left(\left|\partial_{1} w_{\mathrm{v}}\right|^{2}+\left|\operatorname{div}_{\mathrm{v}} w_{\mathrm{v}}\right|^{2}\right) \mathrm{d} x},
$$

and

$$
\begin{aligned}
E(w):= & \int_{\Omega} g\left(\bar{\rho}^{\prime}+\frac{g \bar{\rho}^{2}}{\gamma \bar{P}}\right) w_{3}^{2} \mathrm{~d} x-\int_{\Omega} \frac{1}{\gamma \bar{P}}\left(g \bar{\rho} w_{3}-\gamma \bar{P} \operatorname{div} w\right)^{2} \mathrm{~d} x \\
& -\int_{\Omega} \lambda m^{2}\left(\left|\partial_{1} w_{\mathrm{v}}\right|^{2}+\left|\operatorname{div}_{\mathrm{v}} w_{\mathrm{v}}\right|^{2}\right) \mathrm{d} x,
\end{aligned}
$$

here and in what follows $w_{\mathrm{v}}:=\left(w_{2}, w_{3}\right)$ and $\operatorname{div}_{\mathrm{v}} w_{\mathrm{v}}:=\partial_{2} w_{2}+\partial_{3} w_{3}$ for $w=\left(w_{1}, w_{2}, w_{3}\right)$.

We mention that by virtue of (1.5), $E(w)$ can be rewritten as

$$
\begin{aligned}
E(w)= & -\int_{\Omega} \frac{\lambda g \bar{\rho} m m^{\prime} w_{3}^{2}}{\gamma \bar{P}} \mathrm{~d} x-\int_{\Omega} \frac{1}{\gamma \bar{P}}\left(g \bar{\rho} w_{3}-\gamma \bar{P} \operatorname{div} w\right)^{2} \mathrm{~d} x \\
& -\int_{\Omega} \lambda m^{2}\left(\left|\partial_{1} w_{\mathrm{v}}\right|^{2}+\left|\operatorname{div}_{\mathrm{v}} w_{\mathrm{v}}\right|^{2}\right) \mathrm{d} x .
\end{aligned}
$$

In view of the energy functionals (1.15) and (1.16), we can see that Schwarzschild's condition or the magnetic buoyancy condition may make $E(w)$ to be positive for some $w$, thus contributing to the occurrence of the Parker instability. In particular, if $\Omega$ is an infinite layer domain, Newcomb in 1961 found that Schwarzschild's condition leads to the linear Parker instability in some sense by using the Fourier analysis method [47]. However, in the case of a bounded domain, Jiang et.al. [27] found the stabilizing effect of a strong (equilibrium) magnetic field $\bar{M}$ through a nonslip boundary condition upon the Parker instability, even if the density profile $\bar{\rho}$ satisfies the Schwarzschild's condition. In fact, if we define

$$
\bar{n}:=\sup _{w \in H_{0}^{1}(\Omega)} \sqrt{\frac{\int_{\Omega} g\left(\bar{\rho}^{\prime}+g \bar{\rho}^{2} / \gamma \bar{P}\right) w_{3}^{2} \mathrm{~d} x}{\int_{\Omega} \lambda\left(\left|\partial_{1} w_{\mathrm{v}}\right|^{2}+\left|\operatorname{div}_{\mathrm{v}} w_{\mathrm{v}}\right|^{2}\right) \mathrm{d} x}},
$$

then $\bar{n} \in[0,+\infty)$, since $\Omega$ is bounded in the $x_{1}$-direction. In view of (1.4), for given $\bar{P}, \bar{\rho}, g$ and $\lambda$, we can choose $m$ satisfying $\inf _{x \in \Omega}|m|>\bar{n}$. Then, it is easy to verify that such $m$ satisfies 
$\Xi<0$. This infers that, under the non-slip boundary condition, a sufficiently strong $\bar{M}$ has a remarkable stabilizing effect to prevent the Parker instability from occurring. It is well-known that a magnetic field has the stabilizing effect upon the Parker instability, but can not prevent the Parker instability from occurring in the case of an infinite layer domain. However, the nonslip velocity boundary condition in a bounded domain, imposed in the direction of the magnetic field, can enhance the stabilizing effect of the magnetic field, so that Schwarzschild's criterion (1.7) of the Parker instability fails.

In this article, we further extend the above linear results to the nonlinear case by developing new mathematical techniques. In other words, we will rigorously verify that $\Xi$ is also the discriminant for instability/stability of the nonlinear Parker problem (1.11)-(1.13) under some additional conditions. Moreover, for a horizontally periodic domain, we shall provide a rigorous mathematical proof of the nonlinear Parker instability under Schwarzschild's or Tserkovnikov's condition in the sense of Hadamard. The detailed results of nonlinear stability/instability will be presented in Section 2 .

We end this section by deriving a upper-bound for $\bar{n}$, which may be useful in experimental researches and numerical simulations. From the definition of $\bar{n}$ we see that $\bar{n} \leq \varpi \chi$, where

$$
\varpi:=\sqrt{\left\|g \bar{\rho}^{\prime}+g^{2} \bar{\rho}^{2} / \gamma \bar{P}\right\|_{L^{\infty}(\Omega)} / \lambda} \text { and } \chi:=\sqrt{\frac{\int_{\Omega} w_{3}^{2} \mathrm{~d} x}{\int_{\Omega}\left|\partial_{1} w_{\mathrm{v}}\right|^{2} \mathrm{~d} x}} .
$$

Let $a:=\inf \left\{x_{1} \mid\left(x_{1}, x_{\mathrm{v}}\right) \in \Omega\right\}, b:=\sup \left\{x_{1} \mid\left(x_{1}, x_{\mathrm{v}}\right) \in \Omega\right\}$ and

$$
\Omega^{\prime}:=\left\{\left(x_{1}, x_{\mathrm{v}}\right) \in \mathbb{R}^{3} \mid x_{\mathrm{v}} \in \mathcal{T}, a<x_{1}<b\right\},
$$

where $\mathcal{T}:=\left(2 \pi L_{1} \mathbb{T}\right) \times\left(2 \pi L_{2} \mathbb{T}\right), \mathbb{T}=\mathbb{R} / \mathbb{Z}$, and $2 \pi L_{1}, 2 \pi L_{2}>0$ are the periodicity lengths. Then, one can choose sufficiently large $L_{1}$ and $L_{2}$, such that $H_{0}^{1}(\Omega)$ can be regarded as a subspace of $H\left(\Omega^{\prime}\right)$ by horizontally periodic translation. Thus one has

$$
\bar{n} \leq \varpi \sup _{w \in H_{0}^{1}\left(\Omega^{\prime}\right)} \sqrt{\frac{\int_{\Omega} w_{3}^{2} \mathrm{~d} x}{\int_{\Omega}\left|\partial_{1} w_{\mathrm{v}}\right|^{2} \mathrm{~d} x}} .
$$

On the other hand, similarly to the derivation of [27, Proposition 5.1], we use the Fourier analysis method to infer that

$$
\sup _{w \in H_{0}^{1}\left(\Omega^{\prime}\right)} \sqrt{\frac{\int_{\Omega} w_{3}^{2} \mathrm{~d} x}{\int_{\Omega}\left|\partial_{1} w_{\mathrm{v}}\right|^{2} \mathrm{~d} x}}=\sup _{\psi \in H_{0}^{1}(a, b)} \sqrt{\frac{\int_{a}^{b} \psi^{2} \mathrm{~d} x_{1}}{\int_{a}^{b}\left|\partial_{1} \psi\right|^{2} \mathrm{~d} x_{1}}}
$$

where it is easy to see that

$$
\sup _{\psi \in H_{0}^{1}(a, b)} \sqrt{\frac{\int_{a}^{b} \psi^{2} \mathrm{~d} x_{1}}{\int_{a}^{b}\left|\partial_{1} \psi\right|^{2} \mathrm{~d} x_{1}}}=\frac{b-a}{\pi} .
$$

Consequently, we can deduce from (1.17) $-(1.19)$ that $\bar{n} \leq(b-a) \varpi / \pi$. This means that $\Xi<0$ for $\inf _{x \in \Omega}|m|>(b-a) \varpi / \pi$.

\section{Main results}

In this section we state the main results of this paper on instability/stability of the nonlinear Parker problem (1.11)-(1.13). 


\subsection{Reformulation of the nonlinear stability}

In general, it is difficult to directly show the existence of a unique global-in-time solution to the Parker problem (1.11)-(1.13) defined on a general bounded domain when $\Xi<0$, since the magnetic field is difficult to control. To circumvent such difficulty, similarly to [30, 60], we switch our analysis to that in Lagrangian coordinates. We mention that such transformation method have been also used in the proof of global well-posedness of incompressible MHD equations, please refer to [1, 42, 43, 67].

To show the stability of the nonlinear Parker problem in Lagrangian coordinates, we assume that the domain is a vertical strip, i.e.,

$$
\Omega:=\left\{\left(x_{1}, x_{2}, x_{3}\right) \in \mathbb{R}^{3} \mid x_{1} \in(0, l)\right\} \text { with } l>0 .
$$

To make the expression (1.4) sense, in this article we modify the gravitational constant $g$ to be

$$
0 \leq g:=g\left(x_{3}\right) \in C_{0}^{6}(\mathbb{R})
$$

and suppose that

$$
\bar{\rho} \in B^{7}(\mathbb{R}):=\left\{f \in C^{7}(\mathbb{R})\left|\sup _{x_{3} \in \mathbb{R}}\right| \frac{\mathrm{d}^{i} f}{\mathrm{~d} x^{i}} \mid<\infty \text { for } 0 \leq i \leq 7\right\} .
$$

Under the conditions (2.1) $-(2.3)$ and (1.3) , there always exists a $m$ constructed by (1.4). Moreover, $m$ satisfies

$$
m \in B^{7}(\mathbb{R}) .
$$

We further assume that there is an invertible mapping $\zeta_{0}:=\zeta_{0}(y): \Omega \rightarrow \Omega$, such that

$$
\left.\left(\zeta_{0}-y\right)\right|_{\partial \Omega}=0 \text { and } \operatorname{det}\left(\nabla \zeta_{0}(y)\right) \neq 0 \text { for any } y \in \bar{\Omega} \text {. }
$$

Then, defining the flow map $\zeta$ as the solution to

$$
\left\{\begin{array}{l}
\zeta_{t}(y, t)=v(\zeta(y, t), t) \\
\zeta(y, 0)=\zeta_{0}
\end{array}\right.
$$

we denote the Eulerian coordinates by $(x, t)$ with $x=\zeta(y, t)$, whereas $(y, t) \in \Omega \times \mathbb{R}^{+}$stand for the Lagrangian coordinates. In order to switch back and forth from Lagrangian to Eulerian coordinates, we assume that $\zeta(\cdot, t)$ is invertible and $\Omega=\zeta(\Omega, t)$.

We define now the unknowns in Lagrangian coordinates by

$$
(\sigma, u, B)(y, t)=(\rho, v, M)(\zeta(y, t), t), \quad(y, t) \in \Omega \times \mathbb{R}^{+} .
$$

Thus, the evolution equations for $\sigma, u$ and $B$ in Lagrangian coordinates read as

$$
\left\{\begin{array}{l}
\zeta_{t}=u \\
\sigma_{t}+\sigma \operatorname{div}_{\mathcal{A}} u=0 \\
\sigma u_{t}-\mu_{1} \Delta_{\mathcal{A}} u-\mu_{2} \nabla_{\mathcal{A}} \operatorname{div}_{\mathcal{A}} u+\nabla_{\mathcal{A}}\left(P(\sigma)+\lambda|B|^{2} / 2\right)=\lambda B \cdot \nabla_{\mathcal{A}} B-\sigma \tilde{g} e_{3} \\
B_{t}-B \cdot \nabla_{\mathcal{A}} u+B \operatorname{div}_{\mathcal{A}} u=0 \\
\operatorname{div}_{\mathcal{A}} B=0
\end{array}\right.
$$

with initial and boundary conditions

$$
\left.(u, \zeta-y)\right|_{\partial \Omega}=0 \text { and }\left.(\zeta, \sigma, u, B)\right|_{t=0}=\left(\zeta_{0}, \sigma_{0}, u_{0}, B_{0}\right)
$$


Here $\tilde{g}:=g\left(\zeta_{3}\right)$, the matrix $\mathcal{A}:=\left(\mathcal{A}_{i j}\right)_{3 \times 3}$ via $\mathcal{A}^{T}=(\nabla \zeta)^{-1}:=\left(\partial_{j} \zeta_{i}\right)_{3 \times 3}^{-1}$, and the differential operators $\nabla_{\mathcal{A}}, \operatorname{div} \mathcal{A}$ and $\Delta_{\mathcal{A}}$ are defined by $\nabla_{\mathcal{A}} f:=\left(\mathcal{A}_{1 k} \partial_{k} f, \mathcal{A}_{2 k} \partial_{k} f, \mathcal{A}_{3 k} \partial_{k} f\right)^{T}, \operatorname{div}_{\mathcal{A}}\left(X_{1}, X_{2}, X_{3}\right)^{T}:=$ $\mathcal{A}_{l k} \partial_{k} X_{l}$ and $\Delta_{\mathcal{A}} f:=\operatorname{div}_{\mathcal{A}} \nabla_{\mathcal{A}} f$. It should be noted that we have used the Einstein summation convention over repeated indices, and $\partial_{k}=\partial_{y_{k}}$. Additionally, in view of the definition of $\mathcal{A}$, one can deduce the following two important properties:

$$
\begin{aligned}
& \partial_{l}\left(J \mathcal{A}_{k l}\right)=0, \\
& \partial_{i} \zeta_{k} \mathcal{A}_{k j}=\mathcal{A}_{i k} \partial_{k} \zeta_{j}=\delta_{i j},
\end{aligned}
$$

where $J=\operatorname{det}(\nabla \zeta) \neq 0, \delta_{i j}=0$ for $i \neq j$ and $\delta_{i j}=1$ for $i=j$. The relation (2.7) is often called the geometric identity. In addition, it is easy to check that, by the boundary conditions of $\zeta$ and $\zeta_{0}$,

$$
\left.J \mathcal{A} e_{1}\right|_{\partial \Omega}=\left.J_{0} \mathcal{A}_{0} e_{1}\right|_{\partial \Omega}=e_{1} .
$$

Our next goal is to eliminate $(\sigma, B)$ in $(2.6)$ by expressing them in terms of $\zeta$, and this can be achieved in the same manner as in [30, 60, 66]. We mention that this idea was also used in the proof of the global well-posedness for the Cauchy problem of incompressible or compressible MHD fluids without magnetic diffusivity [1, 21, 22]; please refer to [43, 49, 53, 54] for other relevant results of global well-posedness. For the reader's convenience, we give the derivation here. It follows from $(2.6)_{1}$ that

$$
J_{t}=J \operatorname{div}_{\mathcal{A}} u,
$$

which, together with $(2.6)_{2}$, yields that

$$
\partial_{t}(\sigma J)=0
$$

Applying $J \mathcal{A}_{j l}$ to $(2.6)$, we can use $(2.8)$ and $(2.10)$ to infer that

$$
\begin{aligned}
J \mathcal{A}_{j l} \partial_{t} B_{j} & =J B_{i} \mathcal{A}_{i k}\left(\partial_{t} \partial_{k} \zeta_{j}\right) \mathcal{A}_{j l}-\mathcal{A}_{j l} B_{j} J \operatorname{div}_{\mathcal{A}} u \\
& =-J B_{i} \mathcal{A}_{i k} \partial_{k} \zeta_{j} \partial_{t} \mathcal{A}_{j l}-J_{t} \mathcal{A}_{j l} B_{j}=-J B_{j} \partial_{t} \mathcal{A}_{j l}-J_{t} \mathcal{A}_{j l} B_{j}
\end{aligned}
$$

which implies $\partial_{t}\left(J \mathcal{A}_{j l} B_{j}\right)=0$, i.e.,

$$
\partial_{t}\left(J \mathcal{A}^{T} B\right)=0
$$

In addition, applying div-operator to the above identity and using the geometric identity, we obtain

$$
\partial_{t}\left(J \operatorname{div}_{\mathcal{A}} B\right)=\partial_{t} \operatorname{div}\left(J \mathcal{A}^{T} B\right)=0 .
$$

To obtain the time-asymptotical stability of the equilibrium state, we naturally expect

$$
(\zeta, \sigma, u, B)(t) \rightarrow(y, \bar{\rho}, 0, \bar{M}) \text { as } t \rightarrow \infty
$$

Hence, from (2.7) and (2.11)-(2.13) it follows that

$$
\begin{aligned}
& J \operatorname{div}_{\mathcal{A}} B=J_{0} \operatorname{div}_{\mathcal{A}_{0}} B_{0}=\operatorname{div} \bar{M}=0, \\
& \sigma J=\sigma_{0} J_{0}=\bar{\rho} \text { and } J \mathcal{A}^{T} B=J_{0} \mathcal{A}_{0}^{T} B_{0}=\bar{M},
\end{aligned}
$$

which implies that

$$
\operatorname{div}_{\mathcal{A}} B=0, \quad \sigma=\bar{\rho} J^{-1} \text { and } B=m J^{-1} \partial_{1} \zeta
$$


provided the initial data $\left(\sigma_{0}, B_{0}, \zeta_{0}\right)$ satisfy

$$
\sigma_{0}=\bar{\rho} J_{0}^{-1} \text { and } B_{0}=m J_{0}^{-1} \partial_{1} \zeta_{0} .
$$

Here $\mathcal{A}_{0}$ and $J_{0}$ denote the initial data of $\mathcal{A}$ and $J$, respectively. We mention that, by (2.9) and the fact $J \mathcal{A}^{T} B=J_{0} \mathcal{A}_{0}^{T} B_{0}$, we have $B_{1}=B_{1}^{0}$ on $\partial \Omega$, where $B_{1}^{0}$ is the first component of $B_{0}$.

Let

$$
\eta:=\zeta-y, \text { i.e., } \zeta=\eta+y .
$$

Next we use $\eta$ to represent the (generalized) Lorentz force, the pressure term and the modified gravity term. By a straightforward computation, we can split the Lorentz force into

$$
B \cdot \nabla_{\mathcal{A}} B-\nabla_{\mathcal{A}}|B|^{2} / 2=L o_{1}+\mathcal{N}_{1}-\nabla_{\mathcal{A}}|\tilde{M}|^{2} / 2,
$$

where $\tilde{M}:=\bar{M}\left(\zeta_{3}(y, t)\right)$,

$$
\begin{aligned}
L o_{1}:= & \bar{M} \cdot \nabla(B-\bar{M})+(B-\bar{M}) \cdot \nabla \bar{M}-m m^{\prime} J^{-1} \partial_{1} \eta_{3} e_{1} \\
& -\left(\bar{M}^{T} \nabla(B-\bar{M})+(B-\bar{M})^{T} \nabla \bar{M}\right)^{T}+\nabla\left(m m^{\prime} \eta_{3}\right)
\end{aligned}
$$

and

$$
\begin{aligned}
\mathcal{N}_{1}:= & \bar{M} \cdot\left(\nabla_{\mathcal{A}}-\nabla\right)(B-\bar{M})+(B-\bar{M}) \cdot \nabla_{\mathcal{A}}(B-\bar{M}) \\
& +(B-\bar{M}) \cdot\left(\nabla_{\mathcal{A}}-\nabla\right) \bar{M}-\left(\bar{M}^{T}\left(\nabla_{\mathcal{A}}-\nabla\right)(B-\bar{M})\right. \\
& \left.+(B-\bar{M})^{T} \nabla_{\mathcal{A}}(B-\bar{M})+(B-\bar{M})^{T}\left(\nabla_{\mathcal{A}}-\nabla\right) \bar{M}\right)^{T} \\
& +m m^{\prime} J^{-1}\left(\partial_{1} \eta_{2} \partial_{2} \eta_{3}-\partial_{1} \eta_{3} \partial_{2} \eta_{2}\right) e_{1} \\
& +\left(\nabla_{\mathcal{A}}-\nabla\right)\left(|\tilde{M}|^{2}-|\bar{M}|^{2}\right) / 2+\nabla\left(|\tilde{M}|^{2}-|\bar{M}|^{2}-2 m m^{\prime} \eta_{3}\right) / 2 .
\end{aligned}
$$

On the other hand, by the expression of $B$ in (2.14) and $\bar{M}$, one has

$$
\begin{aligned}
L o_{1} & =m \partial_{1}\left(B-m e_{1}\right)+B_{3} m^{\prime} e_{1}-J^{-1} m m^{\prime} \partial_{1} \eta_{3} e_{1}+\nabla\left(\left(m m^{\prime} \eta_{3}-m\left(B_{1}-m\right)\right)\right. \\
& =m^{2} \partial_{1}\left[J^{-1} \partial_{1} \eta+\left(J^{-1}-1\right) e_{1}\right]+\nabla\left(m m^{\prime} \eta_{3}-m^{2}\left(J^{-1} \partial_{1} \eta_{1}+J^{-1}-1\right)\right) \\
& =\lambda^{-1} \mathcal{L}_{M}+\mathcal{N}_{2},
\end{aligned}
$$

where

$$
\begin{aligned}
\mathcal{L}_{M}= & \lambda\left(m^{2} \partial_{1}^{2} \eta-m^{2} \partial_{1} \operatorname{div} e_{1}+\nabla\left(m m^{\prime} \eta_{3}+m^{2} \operatorname{div}_{\mathrm{v}} \eta_{\mathrm{v}}\right)\right) \\
\mathcal{N}_{2}= & m^{2} \partial_{1}\left(\left(J^{-1}-1\right) \partial_{1} \eta+\left(J^{-1}-1+\operatorname{div} \eta\right) e_{1}\right) \\
& -\nabla\left(m^{2}\left(\left(J^{-1}-1\right) \partial_{1} \eta_{1}+J^{-1}-1+\operatorname{div} \eta\right)\right) .
\end{aligned}
$$

Thus, inserting (2.18) into (2.17), we get

$$
B \cdot \nabla_{\mathcal{A}} B-\nabla_{\mathcal{A}}\left(|B|^{2} / 2\right)=\lambda^{-1} \mathcal{L}_{M}-\nabla_{\mathcal{A}}|\tilde{M}|^{2} / 2+\mathcal{N}_{1}+\mathcal{N}_{2} .
$$

Now we turn to dealing with the pressure term. It is easy to see that

$$
P(\sigma)=P\left(\bar{\rho} J^{-1}\right)=\bar{P}+P^{\prime}(\bar{\rho}) \bar{\rho}\left(J^{-1}-1\right)+\int_{\bar{\rho}}^{\bar{\rho} J^{-1}}\left(\bar{\rho} J^{-1}-z\right) P^{\prime \prime}(z) \mathrm{d} z .
$$

Applying $\nabla_{\mathcal{A}}$-operator to the above identity, we find that

$$
\nabla_{\mathcal{A}} P(\sigma)=\nabla_{\mathcal{A}} P(\tilde{\rho})-\nabla\left(P^{\prime}(\bar{\rho}) \operatorname{div}(\bar{\rho} \eta)\right)-\mathcal{N}_{3},
$$


where $\tilde{\rho}:=\bar{\rho}\left(\zeta_{3}\right)$, and

$$
\begin{aligned}
\mathcal{N}_{3}:= & \left(\nabla-\nabla_{\mathcal{A}}\right)\left(P^{\prime}(\bar{\rho}) \bar{\rho}\left(J^{-1}-1\right)\right)+\nabla\left(P(\tilde{\rho})-\bar{P}-\bar{P}^{\prime} \eta_{3}\right) \\
& +\left(\nabla_{\mathcal{A}}-\nabla\right)(P(\tilde{\rho})-\bar{P})-\nabla\left(P^{\prime}(\bar{\rho}) \bar{\rho}\left(J^{-1}-1+\operatorname{div} \eta\right)\right)-\nabla_{\mathcal{A}} \int_{\bar{\rho}}^{\bar{\rho} J^{-1}}\left(\bar{\rho} J^{-1}-z\right) P^{\prime \prime}(z) \mathrm{d} z .
\end{aligned}
$$

Finally, we represent the modified gravity term as follows.

$$
-\sigma \tilde{g} e_{3}:=g \operatorname{div}(\bar{\rho} \eta) e_{3}-\tilde{\rho} \tilde{g} e_{3}+\mathcal{N}_{4},
$$

where $\mathcal{N}_{4}:=\left(\bar{\rho}\left(1-J^{-1}\right)+\tilde{\rho}-\bar{\rho}\right)(\tilde{g}-g) e_{3}+\left(\tilde{\rho}-\bar{\rho}^{\prime} \eta_{3}-\bar{\rho} J^{-1}-\bar{\rho} \operatorname{div} \eta\right) g e_{3}$.

Summing up the above calculations, we see that, if the initial data $\sigma_{0}, B_{0}, \zeta_{0}$ satisfy (2.15) and (2.5), then we can use the relations (2.19)-(2.21) and (2.16) to transform (2.6) into the following evolution equations for $(\eta, u)$ :

$$
\left\{\begin{array}{l}
\eta_{t}=u, \\
\bar{\rho} J^{-1} u_{t}-\mu_{1} \Delta_{\mathcal{A}} u-\mu_{2} \nabla_{\mathcal{A}} \operatorname{div}_{\mathcal{A}} u-\nabla\left(P^{\prime}(\bar{\rho}) \operatorname{div}(\bar{\rho} \eta)\right)=g \operatorname{div}(\bar{\rho} \eta) e_{3}+\mathcal{L}_{M}+\mathcal{N},
\end{array}\right.
$$

and $(\sigma, B)$ is given by (2.14), where we have utilized the equilibrium state $s_{e}$ in Lagrangian coordinates $\nabla_{\mathcal{A}}\left(P(\tilde{\rho})+\lambda|\tilde{M}|^{2} / 2\right)=-\tilde{\rho} \tilde{g} e_{3}$ for (2.22) , and denoted $\mathcal{A}^{T}=(I+\nabla \eta)^{-1}, I=\left(\delta_{i j}\right)_{3 \times 3}$ and $\mathcal{N}:=\lambda\left(\mathcal{N}_{1}+\mathcal{N}_{2}\right)+\mathcal{N}_{3}+\mathcal{N}_{4}$. The associated initial and boundary conditions read as

$$
\left.(\eta, u)\right|_{t=0}=\left(\eta_{0}, u_{0}\right),\left.\quad(\eta, u)\right|_{\partial \Omega}=0
$$

In this paper, we call the initial-boundary problem (2.22)-(2.23) the transformed Parker problem. Compared with the original Parker problem (1.11)-(1.13), the transformed Parker problem enjoys a fine energy structure, so that one can establish the stabilizing effect of a horizontal magnetic field with the help of non-slip boundary condition by the energy method.

\subsection{Nonlinear stability}

Before stating our main result on the transformed Parker problem, we introduce some notations used throughout this paper. We denote

$$
\begin{aligned}
& \mathbb{R}_{0}^{+}:=[0, \infty), \quad \int:=\int_{\Omega}, \quad L^{p}:=L^{p}(\Omega):=W^{0, p}(\Omega) \text { for } 1<p \leq \infty, \\
& H_{0}^{1}:=W_{0}^{1,2}(\Omega), \quad H^{k}:=W^{k, 2}(\Omega), \quad\|\cdot\|_{k}:=\|\cdot\|_{H^{k}(\Omega)} \text { for } k \geq 0, \\
& \partial_{\mathrm{v}}^{i} \text { deontes } \partial_{2}^{\alpha_{1}} \partial_{3}^{\alpha_{2}} \text { for any } \alpha_{1}+\alpha_{2}=i, \quad\|\cdot\|_{i, k}^{2}:=\sum_{\alpha_{1}+\alpha_{2}=i}\left\|\partial_{2}^{\alpha_{1}} \partial_{3}^{\alpha_{2}} \cdot\right\|_{k}^{2}, \\
& \|f \diamond g\|_{i, k}^{2}:=\sum_{\alpha_{1}+\alpha_{2}=i} \sum_{\beta_{1}+\beta_{2}+\beta_{3} \leq k}\left\|f \partial_{3}^{\beta_{3}} \partial_{1}^{\alpha_{1}+\beta_{1}} \partial_{2}^{\alpha_{2}+\beta_{2}} g\right\|_{0}^{2}, \quad\|f \diamond g\|_{\underline{i}, k}^{2}:=\sum_{0 \leq j \leq i}\|f \diamond g\|_{j, k}^{2}, \\
& \|\cdot\|_{\underline{i}, k}^{2}:=\sum_{0 \leq j \leq i}\|\cdot\|_{j, k}^{2}, \quad a \delta \text { means that } a \leq c b \text { for some constant } c>0, \\
& C_{j}^{j-l} \text { denotes the number of }(j-l) \text {-combinations from a given set } S \text { of } j \text { elements. }
\end{aligned}
$$

The letter $c$ will denote a generic constant which may depend on the domain $\Omega$ and the physical parameters, such as $\lambda, m, g, \mu$ and $\bar{\rho}$ in the original perturbation equations (1.11). It should be noted that a product space $(X)^{n}$ of vector functions is still denoted by $X$, for example, a vector 
function $u \in\left(H^{3}\right)^{3}$ is denoted by $u \in H^{3}$ with norm $\|u\|_{H^{3}}:=\left(\sum_{k=1}^{3}\left\|u_{k}\right\|_{H^{3}}^{2}\right)^{1 / 2}$. Finally, we define some functionals:

$$
\begin{aligned}
& \mathcal{E}^{L}(t):=\|\eta(t)\|_{4}^{2}+\|u(t)\|_{3}^{2}+\left\|u_{t}(t)\right\|_{1}^{2}, \quad \mathcal{E}^{H}(t):=\|\eta(t)\|_{7}^{2}+\sum_{k=0}^{3}\left\|\partial_{t}^{k} u(t)\right\|_{6-2 k}^{2}, \\
& \mathcal{D}^{L}(t):=\left\|\left(\partial_{1} \eta, \operatorname{div} \eta\right)(t)\right\|_{3}^{2}+\sum_{k=0}^{2}\left\|\partial_{t}^{k} u(t)\right\|_{4-2 k}^{2}, \quad \mathcal{D}^{H}(t):=\left\|\left(\partial_{1} \eta, \operatorname{div} \eta\right)(t)\right\|_{6}^{2}+\sum_{k=0}^{3}\left\|\partial_{t}^{k} u(t)\right\|_{7-2 k}^{2}, \\
& \mathcal{G}_{1}(t)=\sup _{0 \leq \tau \leq t} \mathcal{E}^{H}(\tau)+\int_{0}^{t} \mathcal{D}^{H}(\tau) \mathrm{d} \tau, \quad \mathcal{G}_{2}(t)=\sup _{0 \leq \tau \leq t}(1+\tau)^{3} \mathcal{E}^{L}(\tau) .
\end{aligned}
$$

Now, our stability result of the transformed Parker problem reads as follows.

Theorem 2.1. Let $\Omega$ be a vertical strip domain, $(\bar{\rho}, g)$ satisfy (2.3), (2.2) and (1.3), and $m$ be given by (1.4). If $\Xi<0$, then there is a sufficiently small $\delta>0$, such that for any $\left(\eta_{0}, u_{0}\right) \in$ $H^{7} \times H^{6}$ satisfying that

(1) $\left\|\eta_{0}\right\|_{7}^{2}+\left\|u_{0}\right\|_{6}^{2} \leq \delta$

(2) $\zeta_{0}:=y+\eta_{0}$ satisfies (2.5) ;

(3) $\left(\eta_{0}, u_{0}\right)$ satisfies the compatibility conditions on boundary (i.e., $\left.\partial_{t}^{j} u(x, 0)\right|_{\partial \Omega}=0$ for $j=$ $0,1,2)$,

there exists a unique global solution $(\eta, u) \in C\left(\mathbb{R}_{0}^{+}, H^{7} \times H^{6}\right)$ to the transformed Parker problem (2.22)-(2.23). Moreover, $(\eta, u)$ enjoys the following stability estimate:

$$
\mathcal{G}(\infty):=\mathcal{G}_{1}(\infty)+\mathcal{G}_{2}(\infty) \lesssim\left\|\eta_{0}\right\|_{7}^{2}+\left\|u_{0}\right\|_{6}^{2}
$$

Here the constant $\delta$ depends on $\Omega$ and the physical parameters in the original perturbation equations (1.11).

Remark 2.1. Since $\eta=0$ on boundary, then, if $\delta$ in Theorem 2.1 is sufficiently small, we can further have (referring to Lemma 4.2 in [26] for example)

$$
\begin{aligned}
& \zeta:=\eta+y: \bar{\Omega} \rightarrow \bar{\Omega} \text { is a homeomorphism mapping, } \\
& \zeta: \Omega \rightarrow \Omega \text { are } C^{5} \text {-diffeomorphic mapping. }
\end{aligned}
$$

Thus one can recover a stability result in Eulerian coordinates from Theorem 2.1 by an inverse transformation of Lagrangian coordinates, referring to Theorem 1.2 in [28].

Next we briefly describe the basic idea in the proof of Theorem 2.1. By the energy method, there exist two functionals $\tilde{\mathcal{E}}^{L}$ and $\mathcal{Q}$ of $(\eta, u)$ satisfying the lower-order energy inequality for the Parker problem (see Proposition 3.1)

$$
\frac{\mathrm{d}}{\mathrm{d} t} \tilde{\mathcal{E}}^{L}+\mathcal{D}^{L} \leq \mathcal{Q} \mathcal{D}^{L}
$$

in which we can use the stability condition $\Xi<0$ to show that the functional $\tilde{\mathcal{E}}^{L}$ is equivalent to $\mathcal{E}^{L}$. Unfortunately, we can not close the energy estimates only based on (2.27), since $\mathcal{Q}$ can not be controlled by $\tilde{\mathcal{E}}^{L}$. However, we observe that the structure of (2.27) is very similar to the one of the surface wave problem studied in [17, 18], where Guo and Tice developed a two-tier energy 
method to overcome this difficulty. In the spirit of the two-tier energy method, we shall look after a higher-order energy inequality to match the lower-order energy inequality (2.27). Since $\tilde{\mathcal{E}}^{L}$ contains $\|\eta\|_{4}$, we can see that the higher-order energy includes at lest $\|\eta\|_{7}$. Thus, similarly to (2.27), we are able to establish the higher-order energy inequality (see Proposition 4.1):

$$
\frac{\mathrm{d}}{\mathrm{d} t} \tilde{\mathcal{E}}^{H}+\mathcal{D}^{H} \leq \sqrt{\mathcal{E}^{L}}\|\eta\|_{7}^{2}
$$

where the functional $\tilde{\mathcal{E}}^{H}$ is equivalent to $\mathcal{E}^{H}$ by the stability condition. In the derivation of the a priori estimates, we have $\mathcal{Q} \lesssim \mathcal{E}^{H}$, and thus (2.27) implies (see Proposition 3.1)

$$
\frac{\mathrm{d}}{\mathrm{d} t} \tilde{\mathcal{E}}^{L}+\mathcal{D}^{L} \leq 0
$$

Consequently, with the help of the two-tier energy method, (2.28) and (2.29), we can deduce the global-in-time stability estimate (2.24).

We should remark that one of the main novelties in this article lies in that we further develop the stability condition to show that the energy functionals $\tilde{\mathcal{E}}^{L}$ and $\tilde{\mathcal{E}}^{H}$ constructed in (2.27) and (2.28) are equivalent to $\mathcal{E}^{L}$ and $\mathcal{E}^{H}$, respectively. In fact, the energy of the Parker problem includes the term $-E(w)$ for $w=\partial_{\mathrm{v}}^{j} u$ and $\partial_{t}^{j} \eta$. Therefore, we naturally expect that $-E(w)$ should be positive under the stability condition. Otherwise, the two-tier energy method will fail. Exploiting the stability condition and the positivity of the term $\int P^{\prime}(\bar{\rho}) \bar{\rho}|\operatorname{div} w|^{2} \mathrm{~d} y$, we can derive that $-E(w)$ is equivalent to $\left\|\left(w, \partial_{1} w, \operatorname{div}_{\mathrm{v}} w_{\mathrm{v}}, \operatorname{div} w\right)\right\|_{0}^{2}$ (see Lemma 3.4), which plays an important role in the proof of Theorem 2.1.

We mention that the basic idea of the two-tier energy method was also used to show the existence of the global stability solutions to the MHD problem [60], and the stabilizing effect of the magnetic field in the magnetic Rayleigh-Taylor problem [28].

Finally, in view of Theorem 2.1, we immediately obtain the existence of a unique global-intime solution to the original Parker problem, which represents the strong stabilizing effect of the magnetic field through the non-slip boundary condition in the original Parker problem.

Theorem 2.2. Let $\Omega$ be a vertical strip domain, $(\bar{\rho}, g)$ satisfy (2.3), (2.2) and (1.3), and $m$ be given by (1.4). If $\Xi<0$, then there is a sufficiently small $\delta>0$, such that for any $\left(\varrho_{0}, v_{0}, N_{0}\right) \in$ $H^{6}$ satisfying that

(1) there exists an invertible mapping $\zeta_{0}:=\zeta_{0}(x): \Omega \rightarrow \Omega$ such that (2.5);

(2) $\left\|\zeta_{0}-x\right\|_{7}^{2}+\left\|v_{0}\right\|_{6}^{2} \leq \delta$;

(3) $\rho_{0}\left(\zeta_{0}\right)=\bar{\rho}\left(\operatorname{det}\left(\nabla \zeta_{0}\right)\right)^{-1},\left(N_{0}+\bar{M}\right)\left(\zeta_{0}\right)=m\left(\operatorname{det}\left(\nabla \zeta_{0}\right)\right)^{-1} \partial_{1} \zeta_{0}$ and $\operatorname{div}_{\mathcal{A}_{0}}\left(\left(N_{0}+\bar{M}\right)\left(\zeta_{0}\right)\right)=0$, where $\mathcal{A}_{0}^{T}=\left(\nabla \zeta_{0}\right)^{-1}$;

(4) the initial data $\left(\varrho_{0}, v_{0}, N_{0}\right)$ satisfies necessary compatibility conditions (i.e., $\left.\partial_{t}^{j} v(x, 0)\right|_{\partial \Omega}=0$ for $j=0,1,2)$,

there exists a unique global solution $(\varrho, v, N) \in C\left(\mathbb{R}^{+}, H^{6}\right)$ to the original Parker problem (1.11)(1.13). Moreover, $(\varrho, v, N)$ enjoys the following stability estimate:

$$
\begin{aligned}
\sup _{0 \leq t<\infty} & \left(\|(\varrho, N)\|_{6}^{2}+\sum_{k=0}^{3}\left\|\partial_{t}^{k} v(t)\right\|_{6-2 k}^{2}\right) \\
& +\sup _{0 \leq t<\infty}(1+t)^{3}\left(\|(\varrho, v, N)\|_{3}^{2}+\left\|v_{t}\right\|_{1}^{2}\right) \leq\left\|\zeta_{0}-x\right\|_{7}^{2}+\left\|v_{0}\right\|_{6}^{2} .
\end{aligned}
$$

Here the constant $\delta$ depends on $\Omega$ and the physical parameters in the original perturbation equations (1.11). 


\subsection{Nonlinear instability}

As aforementioned, $\Xi$ is the discriminant for the instability/stability of the linearized Parker problem. Next we give an existence result of a local unstable solution to the original Parker problem for $\Xi>0$, which, together with Theorem 2.2 , implies that $\Xi$ is also the discriminant for the instability/stability of the original Parker problem.

Theorem 2.3. Let $\Omega$ be a vertical strip domain, $(\bar{\rho}, g)$ satisfy (2.3), (2.2) and (1.3), and $m$ be given by (1.4). If $\Xi>0$, then the Parker problem (1.11)-(1.13) is unstable, that is, there are positive constants $\varepsilon$ and $\iota$, and a quaternion $\left(\tilde{\varrho}_{0}, \tilde{v}_{0}, \tilde{N}_{0}, v_{\mathrm{r}}\right) \in H^{3}$, such that for any $\delta \in(0, \iota)$ and the initial data $\left(\varrho_{0}, v_{0}, N_{0}\right):=\delta\left(\tilde{\varrho}_{0}, \tilde{v}_{0}, \tilde{N}_{0}\right)+\left(0, \delta^{2} v_{\mathrm{r}}, 0\right)$, there is a unique classical solution $(\varrho, v, N)$ of the Parker problem (1.11) -(1.13) on $\left[0, T^{\mathrm{max}}\right)$, but

$$
\|(\varrho, u, N)(T)\|_{3} \geq \varepsilon \quad \text { for some time } T \in\left(0, T^{\max }\right)
$$

where $\left(\varrho_{0}, v_{0}, N_{0}\right)$ satisfies the compatibility conditions $\left.\partial_{t}^{i} v(x, 0)\right|_{\partial \Omega}=0(i=0,1)$ and $\operatorname{div} N_{0}=0$, $T^{\max }$ denotes the maximal time of existence of the solution $(\varrho, v, N)$, and $\varepsilon$, $\iota$, and $\left(\tilde{\varrho}_{0}, \tilde{v}_{0}, \tilde{N}_{0}, v_{\mathrm{r}}\right)$ depend on $\Omega$ and the physical parameters in the original perturbation equations (1.11).

Theorem 2.3 reveals that the Parker instability will occur for $\Xi>0$. Roughly speaking, Theorem 2.3 is proved based on a new version of the bootstrap method. The method of bootstrap instability probably started from Guo and Strauss' works [15, 16]. Then, various versions of the bootstrap method have been developed in the study of dynamical instability of various physical models, please refer to [10 12, 14, 23, 25] for more details. Unfortunately, the known versions of the bootstrap method can not be directly applied to the Parker problem here due to the presence of the magnetic field and non-slip boundary condition. In this paper we develop a new version of the bootstrap method to overcome such difficulties and establish the Parker instability. The basic idea of our new bootstrap method can be found after Lemma 6.3, and the key technique mainly lies in the derivation of the error estimate (6.20) in Lemma 6.2, which is obtained by exploiting the structure of the original perturbation equations. Moreover, we develop an approach based on the elliptic theory to construct the compatible initial data of the nonlinear unstable solution by using the initial data of the linear unstable solution, see Lemma 6.1. We mention that Theorem 2.3 also holds for a bounded $C^{4}$-domain by applying our new bootstrap method, and moreover, the smoothness requirements (2.2) $-(\underline{2.4})$ can be relaxed.

\subsection{Horizontally periodic domains}

Now we further consider the case of a horizontally periodic domain, i.e.,

$$
\Omega:=\left\{x=\left(x_{h}, x_{3}\right) \in \mathbb{R}^{3} \mid x_{h} \in \mathcal{T},-l<x_{3}<l\right\} \quad \text { with } l>0,
$$

where $x_{h}:=\left(x_{1}, x_{2}\right), \mathcal{T}:=\left(2 \pi L_{1} \mathbb{T}\right) \times\left(2 \pi L_{2} \mathbb{T}\right), \mathbb{T}=\mathbb{R} / \mathbb{Z}$, and $2 \pi L_{1}, 2 \pi L_{2}>0$ are the periodicity lengths. Then we can verify that $\Xi>0$ under Schwarzschild's condition with sufficiently large $L_{1}$ or Tserkovnikov's condition. The detailed verification will be presented in Section 7 . Hence, we can follow the proof of Theorem 2.3 to establish the nonlinear Parker instability under Schwarzschild's or Tserkovnikov's condition.

Before stating our nonlinear Parker instability results in a horizontally periodic domain, we 
introduce some notations:

$$
\begin{aligned}
& \chi(\psi):=\int_{-l}^{l}\left(\lambda m^{2}\left(\left|\psi^{\prime}\right|^{2}+\frac{\psi^{2}}{L_{2}^{2}}\right)-\left(\frac{g^{2} \bar{\rho}^{2}}{\gamma \bar{P}}+g \bar{\rho}^{\prime}\right) \psi^{2}\right) \mathrm{d} x_{3}, \\
& \xi_{3 D}:=\sup _{\psi \in H_{0}^{1}(-l, l)} \sqrt{\frac{\sqrt{\chi^{2}(\psi)+\frac{4}{L_{2}^{2}} \int_{-l}^{l} \lambda m^{2} \psi^{2} \mathrm{~d} x_{3} \int_{-l}^{l}\left(\frac{g^{2} \bar{\rho}^{2}}{\gamma P}+g \bar{\rho}^{\prime}\right) \psi^{2} \mathrm{~d} x_{3}}-\chi(\psi)}{2 \int_{-l}^{l} \lambda m^{2} \psi^{2} \mathrm{~d} x_{3}}} \\
& \kappa(l):=\sup _{\psi \in H_{0}^{1}(-l, l)} \sqrt{\frac{\int_{-l}^{l}\left(\frac{g^{2} \bar{\rho}^{2}}{\gamma \bar{P}}+g \bar{\rho}^{\prime}\right) \psi^{2} \mathrm{~d} x_{2}}{\int_{-l}^{l} \lambda m^{2}\left|\psi^{\prime}\right|^{2} \mathrm{~d} x_{2}},} \\
& \xi_{2 D}:=\sup _{\psi \in H_{0}^{1}(-l, l)} \sqrt{\frac{\int_{-l}^{l}\left(\left(\frac{g^{2} \bar{\rho}^{2}}{\gamma \bar{P}}+g \bar{\rho}^{\prime}\right) \psi^{2}-\lambda m^{2}\left|\psi^{\prime}\right|^{2}\right) \mathrm{d} x_{2}}{\int_{-l}^{l} \lambda m^{2} \psi^{2} \mathrm{~d} x_{2}}}
\end{aligned}
$$

where we have omitted to mention that $\psi \in H_{0}^{1}(-l, l)$ should make the denominators and square roots sense in the definitions of $\xi_{3 D}$ and $\xi_{2 D}$. Next we state the main results.

Theorem 2.4. Let $l \in(0, \infty)$, $\Omega$ be defined by (2.32), $g$ be a constant, $\bar{\rho}:=\bar{\rho}\left(x_{3}\right) \in C^{4}(\bar{\Omega})$ satisfy (1.3), and $m$ be given by (1.4). If one of the following two assumptions holds

(1) $\bar{\rho}$ satisfies Schwarzschild's condition (1.7), and $L_{1}>\xi_{3 D}^{-1}$;

(2) $\bar{\rho}$ satisfies Tserkovnikov's condition (1.8);

then, the Parker problem (1.11) -(1.13) is unstable as in Theorem 2.3.

Remark 2.2. By virtue of Schwarzschild's condition, there exists an open interval $I \subset(-l, l)$, such that

$$
-\bar{\rho}^{\prime}<\frac{g \bar{\rho}^{2}}{\gamma \bar{P}} \quad \text { for any } x_{3} \in I .
$$

Then we choose a function $\psi_{0} \in H_{0}^{1}(-l, l)$ such that

$$
\psi_{0}\left(x_{3}\right)>0 \text { in } I \text { and } \psi_{0}\left(x_{3}\right)=0 \text { in }(-l, l) \backslash I \text {, }
$$

whence,

$$
\int_{-l}^{l}\left(\frac{g^{2} \bar{\rho}^{2}}{\gamma \bar{P}}+g \bar{\rho}^{\prime}\right) \psi_{0}^{2} \mathrm{~d} x_{3}>0,
$$

which implies that $\xi_{3 D}$ must be a positive constant. Moreover, for given $\bar{\rho}$, we have

$$
\xi_{3 D} \rightarrow 0 \quad \text { as }|m| \rightarrow \infty .
$$

Remark 2.3. It should be noted that Schwarzschild's condition is equivalent to the magnetic buoyancy condition. Hence, Theorem 2.4 tells us that if there is a point, at which the magnetic buoyancy points oppositely to the direction of the gravity field in the equilibrium state $s_{e}$, then the Parker problem is unstable. If the direction of the magnetic buoyancy is in line everywhere with the direction of the gravity field in the equilibrium state, i.e., Schwarzschild's condition fails, then we have $\Xi<0$ for a vertical strip domain with non-slip boundary condition, which immediately implies that the Parker problem is stable by virtue of Theorem 2.2. We can observe that the proof of Theorem 2.2 strongly depends on the non-slip condition in the horizontal direction. A question arises whether this stability conclusion in Theorem 2.2 can be generalized to a horizontally periodic domain. We shall further investigate this question in a separate article. 
Remark 2.4. If $l=\infty$ and $g \in C_{0}^{3}(\mathbb{R})$, we can establish a Gronwall-type inequality as (3.39) in [29, Proposition 3.2] for the classical solution $(\varrho, u, N) \in C^{0}\left([0, T), H^{3}\right)$ of the Parker problem. Thus we can deduce a more precise result on the nonlinear Parker instability for both 2D and 3D cases by a standard bootstrap method, in which the third component of the velocity is unstable in $L^{2}$ as (1.10) in [29, Theorem 1.1].

Similarly, we can also establish the following nonlinear Parker instability result in the 2D case.

Theorem 2.5. Let $l \in(0, \infty), \Omega:=2 \pi L_{1} \mathbb{T} \times(-l, l) \subset \mathbb{R}^{2}, g$ be a constant, $\bar{\rho}:=\bar{\rho}\left(x_{2}\right) \in C^{4}(\Omega)$ satisfy (1.3), and $m$ be given by (1.4). If $\kappa>1$ and $L_{1}>\xi_{2 D}^{-1}$, then the Parker problem (1.12) (1.14) in the 2D case is unstable as in Theorem 2.4.

Remark 2.5. It is interesting to notice that the definition of $\kappa$ is very similar to the critical number $M_{\mathrm{c}}(l)$ of the 3D incompressible magnetic RT problem around a vertical equilibrium magnetic field, where

$$
M_{\mathrm{c}}(l):=\sqrt{\sup _{\psi \in H_{0}^{1}(-l, l)} \frac{g \int_{-l}^{l} \bar{\rho}^{\prime}|\psi|^{2} \mathrm{~d} s}{\lambda \int_{-l}^{l}\left|\psi^{\prime}\right|^{2} \mathrm{~d} s}}>0 \text { for } \bar{\rho}^{\prime}(s)>0 \text { for some } s \in(-l, l)
$$

and $M_{\mathrm{c}}(l)<\infty$ for $l<\infty$, please refer to [29, Theorem 1.1]. Moreover, $M_{\mathrm{c}}(l)$ is also the critical number $M_{\mathrm{c}}(l)$ of the $2 \mathrm{D}$ incompressible magnetic RT problem around a horizontal equilibrium magnetic field, please refer to [65].

Remark 2.6. Under Schwarzschild's condition, we can have that

$$
0<\kappa(l)<\infty \text { for } l<\infty \text {. }
$$

Moreover, $\kappa \rightarrow 0$ as $m \rightarrow \infty$ for a given $\bar{\rho}$. On the other hand, if $\kappa \in(0,1]$, we can use the Fourier analysis method to obtain $E(w) \leq 0$ for any $w \in H_{0}^{1}$, which implies $\Xi \leq 0$. Thus we see that a sufficiently large horizontal magnetic field has the stabilizing effect in the $2 \mathrm{D}$ case. This conclusion agrees with the result for the $2 \mathrm{D}$ incompressible magnetic RT problem around a horizontal equilibrium magnetic field. In addition, if $l=\infty$ and $0 \leq g \in C_{0}^{0}(\mathbb{R})$, under Schwarzschild's condition, we can derive that

$$
\kappa(\infty)= \begin{cases}\text { infinity, } & \text { if } \int_{\mathbb{R}}\left(\frac{g^{2} \bar{\rho}^{2}}{\gamma \bar{P}}+g \bar{\rho}^{\prime}\right) \mathrm{d} x_{2}>0 ; \\ \text { a positive real number, } & \text { if } \int_{\mathbb{R}}\left(\frac{g^{2} \bar{\rho}^{2}}{\gamma \bar{P}}+g \bar{\rho}^{\prime}\right) \mathrm{d} x_{2}<0,\end{cases}
$$

please refer to [29, Proposition 2.1] for the proof.

The rest sections are mainly devoted to the proof of Theorems 2.1 2.5. In Section 3 we first derive the lower-order energy inequality (2.29) of the transformed Parker problem. Then in Section 4, we derive the higher-order energy inequality (2.28). Finally, based on the previous two energy inequalities, we show Theorem 2.1 by applying the two-tier energy method, and further deduce Theorem 2.2 from Theorem 2.1 in Section 5. In Section 6, we develop a new version of the bootstrap method to prove Theorem 2.3 . Finally, we verify that $\Xi>0$ under the assumptions of Theorem 2.4 2.5 in Section 7. 


\section{Lower-order energy inequality}

In this section we derive the lower-order energy inequality for the transformed Parker problem. To this end, let $(\eta, u)$ be a solution of the transformed Parker problem, such that

$$
\sqrt{\sup _{0 \leq \tau \leq T} \mathcal{E}^{H}(\tau)} \leq \delta \in(0,1) \text { for some } T>0,
$$

where $\delta$ is sufficiently small. It should be noted that the smallness depends on the domain $\Omega$ and the physical parameters in the perturbation equations (1.11). Moreover, we assume that the solution $(\eta, u)$ possesses proper regularity, so that the procedure of formal calculations makes sense. In the calculations that follow, we shall repeatedly use Cauchy-Schwarz's inequality, Hölder's inequality, and the embedding inequalities (see [3, 4.12 Theorem])

$$
\begin{aligned}
& \|f\|_{L^{p}} \lesssim\|f\|_{1} \quad \text { for } 2 \leq p \leq 6, \\
& \|f\|_{L^{\infty}} \lesssim\|f\|_{2} .
\end{aligned}
$$

and the interpolation inequality in $H^{j}$ (see [3, 5.2 Theorem])

$$
\|f\|_{j} \lesssim\|f\|_{0}^{1-\frac{j}{i}}\|f\|_{i}^{\frac{j}{i}} \leq C_{\epsilon}\|f\|_{0}+\epsilon\|f\|_{j+1}
$$

for any $0 \leq j<i$ and any constant $\epsilon>0$, where the constant $C_{\epsilon}$ depends on $\Omega$ and $\epsilon$. In addition, we shall also repeatedly use the following two estimates:

$$
\|f g\|_{j} \lesssim \begin{cases}\|f\|_{1}\|g\|_{1} & \text { for } j=0 \\ \|f\|_{j}\|g\|_{2} & \text { for } 0 \leq j \leq 2 \\ \|f\|_{2}\|g\|_{j}+\|f\|_{j}\|g\|_{2} & \text { for } 3 \leq j \leq 5 \\ \|f\|_{2}\|g\|_{j}+\|f\|_{5}\|g\|_{j-3}+\|f\|_{j}\|g\|_{2} & \text { for } 6 \leq j \leq 7\end{cases}
$$

and

$$
\|f\|_{0} \lesssim\left\|\partial_{1} f\right\|_{0} \text { for } f \in H_{0}^{1}
$$

where (3.5) can be easily verified by Hölder's inequality and the embedding inequalities (3.2) (3.3).

Before deriving the lower-order energy inequality defined on $(0, T]$, we first give some preliminary estimates, temporal derivative estimates, $y_{\mathrm{v}}$-derivative estimates (i.e., the estimates of partial derivatives with respect to $y_{2}$ and $\left.y_{3}\right)$ and $y$-derivative estimates (i.e., the estimates of partial derivatives with respect to $y_{1}, y_{2}$ and $\left.y_{3}\right)$ in sequence.

\subsection{Preliminary estimates}

In this subsection we introduce some preliminary estimates on $J, J^{-1}, \mathcal{A}$ and $\mathcal{N}$, which will be repeatedly used in estimating $(\eta, u)$ later.

Lemma 3.1. The following estimates hold.

$$
\begin{aligned}
& 1 \lesssim\|J\|_{L^{\infty}} \lesssim 1 \\
& \|J-1\|_{l} \lesssim\|\eta\|_{l+1}, \\
& \left\|\partial_{t}^{i} J\right\|_{j} \lesssim \sum_{k=0}^{i-1}\left\|\partial_{t}^{k} u\right\|_{j+1}, \\
& 1 \lesssim\left\|J^{-1}\right\|_{L^{\infty}} \lesssim 1 \\
& \left\|J^{-1}-1\right\|_{l} \lesssim\|\eta\|_{l+1}, \\
& \left\|\partial_{t}^{i} J^{-1}\right\|_{j} \lesssim \sum_{k=0}^{i-1}\left\|\partial_{t}^{k} u\right\|_{j+1}
\end{aligned}
$$


where $0 \leq l \leq 6,1 \leq i \leq 3$ and $0 \leq j \leq 7-2 i$.

Proof. Recalling the definition of $J=\operatorname{det}(\nabla \eta+I)$ and using the expansion theorem of determinants, we find that

$$
J=1+\operatorname{div} \eta+P_{2}(\nabla \eta)+P_{3}(\nabla \eta),
$$

where $P_{i}(\nabla \eta)(i=2,3)$ denotes the homogeneous polynomial of degree $i$ with respect to $\partial_{j} \eta_{k}$ for $1 \leq j, k \leq 3$. Using (3.5), (3.3), and the smallness condition (3.1), we immediately get (3.8) and (3.7). Similarly, we easily obtain (3.9) from (3.13) and (2.22) 1 .

By (3.13) and (3.7), we have (3.10) and $J^{-1}=\left(1+\operatorname{div} \eta+P_{2}(\nabla \eta)+P_{3}(\nabla \eta)\right)^{-1}$, which implies

$$
J^{-1}-1=-\left(J^{-1}-1\right)\left(\operatorname{div} \eta+P_{2}(\nabla \eta)+P_{3}(\nabla \eta)\right)-\left(\operatorname{div} \eta+P_{2}(\nabla \eta)+P_{3}(\nabla \eta)\right) .
$$

Thus, we obtain (3.11) by using (3.5) and the smallness condition.

Finally, noting that

$$
\begin{aligned}
& J_{t}^{-1}=-J^{-2} J_{t}, \quad J_{t t}^{-1}=2 J^{-3} J_{t}^{2}-J^{-2} J_{t t}, \quad J_{t t t}^{-1}=-6 J^{-4} J_{t}^{3}+6 J^{-3} J_{t} J_{t t}-J^{-2} \partial_{t}^{3} J \\
& \left\|J^{-1} \psi\right\|_{l} \lesssim\left\|\left(J^{-1}-1\right) \psi\right\|_{l}+\|\psi\|_{l} \lesssim\|\psi\|_{l} \quad \text { for } \quad 0 \leq l \leq 6,
\end{aligned}
$$

we make use of (3.9) and (3.5) to get (3.12) immediately. This completes the proof.

Lemma 3.2. It holds that

$$
\begin{aligned}
& \|\mathcal{A}\|_{L^{\infty}} \lesssim 1 \\
& \left\|\partial_{t}^{i} \mathcal{A}\right\|_{j} \lesssim \sum_{k=0}^{i-1}\left\|\partial_{t}^{k} u\right\|_{j+1} \\
& \|\tilde{\mathcal{A}}\|_{l} \lesssim\|\eta\|_{l+1}
\end{aligned}
$$

where $\tilde{\mathcal{A}}:=(\mathcal{A}-I), 0 \leq l \leq 6,1 \leq i \leq 3$ and $0 \leq j \leq 7-2 i$.

Proof. Recalling the definition of $\mathcal{A}$, we see that $\mathcal{A}=\left(A_{k l}^{*}\right)_{3 \times 3} J^{-1}$, where $A_{k l}^{*}$ is the algebraic complement minor of $(k, l)$-th entry in the matrix $\nabla \eta+I$ and the polynomial of degree 1 or 2 with respect to $\partial_{m} \eta_{n}$ for $1 \leq m, n \leq 3$. Thus, employing (3.10), (3.3) and the smallness condition, we obtain (3.14).

Using (3.11), (3.5), (2.22) 1 and the smallness condition, we have

$$
\begin{aligned}
& \left\|\partial_{t}^{i} A_{k l}^{*}\right\|_{j} \lesssim \sum_{m=0}^{i-1}\left\|\partial_{t}^{m} u\right\|_{j+1}, \quad\left\|A_{k l}^{*} \partial_{t}^{i} J^{-1}\right\|_{j} \lesssim\left(1+\|\eta\|_{6}\right)\left\|\partial_{t}^{i} J^{-1}\right\|_{j} \lesssim\left\|\partial_{t}^{i} J^{-1}\right\|_{j}, \\
& \left\|\partial_{t}^{i} A_{k l}^{*} J^{-1}\right\|_{j} \lesssim\left\|\partial_{t}^{i} A_{k l}^{*}\left(J^{-1}-1\right)\right\|_{j}+\left\|\partial_{t}^{i} A_{k l}^{*}\right\|_{j} \lesssim\left\|\partial_{t}^{i} A_{k l}^{*}\right\|_{j}, \quad 1 \leq i \leq 3,0 \leq j \leq 7-2 i .
\end{aligned}
$$

Thus we can further make use of the above three estimates, (3.12) and (3.5) to deduce that

$$
\left\|\partial_{t}^{i} \mathcal{A}\right\|_{j}=\left\|\sum_{m=0}^{i} C_{i}^{m}\left(\partial_{t}^{i-m} A_{k l}^{*}\right)_{3 \times 3} \partial_{t}^{m} J^{-1}\right\|_{j} \lesssim \sum_{k=0}^{i-1}\left\|\partial_{t}^{k} u\right\|_{j+1},
$$

which yields (3.15). 
We proceed to evaluating $\tilde{\mathcal{A}}$. Since $\delta$ is assumed to be so small that the following power series holds.

$$
\mathcal{A}^{T}=I-\nabla \eta+(\nabla \eta)^{2} \sum_{i=0}^{\infty}(-\nabla \eta)^{i}=I-\nabla \eta+(\nabla \eta)^{2} \mathcal{A}^{T}
$$

whence,

$$
\tilde{\mathcal{A}}^{T}=(\nabla \eta)^{2} \mathcal{A}^{T}-\nabla \eta=(\nabla \eta)^{2} \tilde{\mathcal{A}}^{T}+(\nabla \eta)^{2}-\nabla \eta .
$$

Hence, we can use (3.5) to deduce (3.16) from (3.17) for sufficiently small $\delta$. The proof is complete.

Lemma 3.3. It holds that

$$
\begin{aligned}
& \|\mathcal{N}\|_{j} \lesssim\|\eta\|_{j-1}\|\eta\|_{7} \quad \text { for } j=3 \text { and } 5 \\
& \left\|\mathcal{N}_{t}\right\|_{2} \lesssim\|\eta\|_{4}\|u\|_{4} \\
& \left\|\mathcal{N}_{t t}\right\|_{0} \lesssim\|\eta\|_{4}\left(\|u\|_{2}+\left\|u_{t}\right\|_{2}\right)+\|u\|_{3}^{2} \\
& \left\|\mathcal{N}_{t}\right\|_{3}+\left\|\mathcal{N}_{t t}\right\|_{1}+\left\|\partial_{t}^{3} \mathcal{N}\right\|_{0} \lesssim \sqrt{\mathcal{E}^{L} \mathcal{D}^{H}}
\end{aligned}
$$

Proof. Since $\mathcal{N}$ is a linear combination of $\mathcal{N}_{i}$ for $i=1, \ldots$, it suffices to verify the above estimates with $\mathcal{N}_{i}$ in places of $\mathcal{N}$. By a straightforward calculation, we find that

$$
\begin{aligned}
& \nabla_{\mathcal{A}}-\nabla=\nabla_{\tilde{\mathcal{A}}}, \\
& B-\bar{M}=m\left(\left(J^{-1}-1\right) \partial_{1} \eta+\partial_{1} \eta+\left(J^{-1}-1\right) e_{1}\right) \\
& |\tilde{M}|^{2}-\bar{M}^{2}=2 \int_{0}^{\eta_{3}}\left(m m^{\prime}\right)\left(s+y_{3}\right) \mathrm{d} s \\
& |\tilde{M}|^{2}-\bar{M}^{2}-2 m m^{\prime} \eta_{3}=2 \int_{0}^{\eta_{3}} \int_{0}^{s}\left(m m^{\prime}\right)^{\prime}\left(\tau+y_{3}\right) \mathrm{d} \tau \mathrm{d} s \\
& J^{-1}-1+\operatorname{div} \eta=J^{-1}\left((J-1) \operatorname{div} \eta-P_{2}(\nabla \eta)-P_{3}(\nabla \eta)\right) \\
& P(\tilde{\rho})-\bar{P}=\int_{0}^{\eta_{3}} \frac{\mathrm{d}}{\mathrm{d} y_{3}} P\left(\bar{\rho}\left(s+y_{3}\right)\right) \mathrm{d} s \\
& P(\tilde{\rho})-\bar{P}-\bar{P}^{\prime} \eta_{3}=\int_{0}^{\eta_{3}} \int_{0}^{s} \frac{\mathrm{d}^{2}}{\mathrm{~d} y_{3}^{2}} P\left(\bar{\rho}\left(\tau+y_{3}\right)\right) \mathrm{d} \tau \mathrm{d} s, \\
& \int_{\bar{\rho}}^{\bar{\rho} J^{-1}} \quad\left(\bar{\rho} J^{-1}-z\right) P^{\prime \prime}(z) \mathrm{d} z=\int_{0}^{\bar{\rho}\left(J^{-1}-1\right)}\left(\bar{\rho}\left(J^{-1}-1\right)-z\right) P^{\prime \prime}(z+\bar{\rho}) \mathrm{d} z, \\
& \tilde{\rho}-\bar{\rho}^{\prime} \eta_{3}-\bar{\rho}^{-1}-\bar{\rho} \operatorname{div} \eta \\
& =\int_{0}^{\eta_{3}} \int_{0}^{s} \bar{\rho}^{\prime \prime}\left(\tau+y_{3}\right) \mathrm{d} \tau \mathrm{d} s+\bar{\rho}\left(J^{-1}-1\right) \operatorname{div} \eta+\bar{\rho} J^{-1}\left(P_{2}(\nabla \eta)+P_{3}(\nabla \eta)\right) .
\end{aligned}
$$

Putting the above relations into the expressions of $\mathcal{N}_{1}-\mathcal{N}_{4}$, we use Lemmas 3.1 3.2, (3.4) - (3.5) and (2.2)-(2.4) to see that (3.18)-(3.21) hold with $\mathcal{N}_{i}$ in place of $\mathcal{N}$ for $i=1, \ldots$, 4. This completes the proof.

Finally, we derive an important estimate from the stability condition.

Lemma 3.4. If $\Xi<0$, then

$$
\left\|\left(w, \partial_{1} w, \operatorname{div}_{\mathrm{v}} w_{\mathrm{v}}, \operatorname{div} w\right)\right\|_{0}^{2} \lesssim-E(w),
$$

where $E(w)$ is defined by (1.15). 
Proof. Since $\Xi<0$, one has $-\Xi \lambda\left\|\left(\partial_{1} w_{\mathrm{v}}, \operatorname{div}_{\mathrm{v}} w_{\mathrm{v}}\right)\right\|_{0}^{2} \leq-E(w)$, which, together with (3.6), yields

$$
\left\|\left(w_{\mathrm{v}}, \partial_{1} w_{\mathrm{v}}, \operatorname{div}_{\mathrm{v}} w_{\mathrm{v}}\right)\right\|_{0}^{2} \lesssim-E(w) .
$$

On the other hand, by virtue of (1.3),

$$
\begin{aligned}
\|\operatorname{div} w\|_{0}^{2} & \lesssim \int\left(P^{\prime}(\bar{\rho}) \bar{\rho}|\operatorname{div} w|^{2}+\lambda m^{2}\left(\left|\partial_{1} w_{\mathrm{v}}\right|^{2}+\left|\operatorname{div}_{\mathrm{v}} w_{\mathrm{v}}\right|^{2}\right)\right) \mathrm{d} x \\
& =\int g \bar{\rho}^{\prime} w_{3}^{2} \mathrm{~d} x+\int 2 g \bar{\rho} \operatorname{div} w w_{3} \mathrm{~d} x-E(w),
\end{aligned}
$$

which, combined with (2.2), (2.3) and the relation

$$
\operatorname{div} w=\partial_{1} w_{1}+\operatorname{div}_{\mathrm{v}} w_{\mathrm{v}}
$$

results in

$$
c_{1}\left\|\left(\partial_{1} w_{1}, \operatorname{div} w\right)\right\|_{0}^{2} \leq c_{2}\left\|\left(w_{3}, \operatorname{div}_{\mathrm{v}} w_{\mathrm{v}}\right)\right\|_{0}^{2}-E(w),
$$

where the positive constants $c_{1}$ and $c_{2}$ depend on $\Omega$ and the physical parameters. Therefore, the desired conclusion follows from (3.24), (3.22) and (3.6) immediately.

\subsection{Temporal derivative estimates}

In this subsection we establish the estimates of temporal derivatives. To this end, we apply $\partial_{t}^{j}$ to (2.22) to get

$$
\left\{\begin{array}{l}
\partial_{t}^{j+1} \eta=\partial_{t}^{j} u \\
\bar{\rho} J^{-1} \partial_{t}^{j+1} u-\mu_{1} \Delta_{\mathcal{A}} \partial_{t}^{j} u-\mu_{2} \nabla_{\mathcal{A}} \operatorname{div}_{\mathcal{A}} \partial_{t}^{j} u-\nabla\left(P^{\prime}(\bar{\rho}) \operatorname{div}\left(\bar{\rho} \partial_{t}^{j} \eta\right)\right) \\
\quad=g \operatorname{div}\left(\bar{\rho} \partial_{t}^{j} \eta\right) e_{3}+\partial_{t}^{j} \mathcal{L}_{M}+\partial_{t}^{j} \mathcal{N}+N_{u}^{t, j}
\end{array}\right.
$$

where

$$
\begin{aligned}
N_{u}^{t, j}:= & \sum_{0 \leq k<j, 0 \leq l \leq j}\left(\mu_{1} C_{j}^{k+l} C_{k+l}^{k} \partial_{t}^{j-k-l} \mathcal{A}_{i l} \partial_{l}\left(\partial_{t}^{l} \mathcal{A}_{i k} \partial_{t}^{k} \partial_{k} u\right)\right. \\
& \left.\quad+\mu_{2} C_{j}^{k+l} C_{k+l}^{k}\left(\partial_{t}^{j-k-l} \mathcal{A}_{i l} \partial_{l}\left(\partial_{t}^{l} \mathcal{A}_{s k} \partial_{t}^{k} \partial_{k} u_{s}\right)\right)_{3 \times 1}\right)-\sum_{k=0}^{j-1} \bar{\rho} \partial_{t}^{j-k} J^{-1} \partial_{t}^{k+1} u
\end{aligned}
$$

Moreover, making use of (‥16), (3.15), (3.12), (3.5), and interpolation inequality, we easily infer the following estimates on $N_{u}^{t, j}$ :

$$
\begin{aligned}
\left\|N_{u}^{t, 1}\right\|_{2} & \lesssim\|u\|_{3}\left(\|u\|_{4}+\left\|u_{t}\right\|_{2}\right), \\
\left\|N_{u}^{t, 2}\right\|_{0} & \lesssim\|u\|_{3}^{2}+\left(\|u\|_{3}+\left\|u_{t}\right\|_{1}\right)\left\|u_{t}\right\|_{2}+\|u\|_{3}\left\|u_{t t}\right\|_{0}, \\
\left\|N_{u}^{t, 3}\right\|_{0} & \lesssim \sqrt{\mathcal{E}^{L}} \mathcal{D}^{H}+\left\|u_{t}\right\|_{3}^{2} \lesssim \sqrt{\mathcal{E}^{L}} \mathcal{D}^{H}
\end{aligned}
$$

Then we can further deduce the following estimate.

Lemma 3.5. It holds that

$$
\frac{\mathrm{d}}{\mathrm{d} t}\left\|\left(\sqrt{\mu_{1} J} \nabla_{\mathcal{A}} u_{t}, \sqrt{\mu_{2} J} \operatorname{div}_{\mathcal{A}} u_{t}\right)\right\|_{0}^{2}+c\left\|u_{t t}\right\|_{0}^{2} \lesssim\|u\|_{2}^{2}+\sqrt{\mathcal{E}^{H}} \mathcal{D}^{L} .
$$


ProOF. Multiplying (3.25) 2 with $j=1$ by $J u_{t t}$, integrating (by parts) the resulting equality over $\Omega$ and using (2.7), we obtain

$$
\begin{aligned}
& \frac{1}{2} \frac{\mathrm{d}}{\mathrm{d} t} \int J\left(\mu_{1}\left|\nabla_{\mathcal{A}} u_{t}\right|^{2}+\mu_{2}\left|\operatorname{div}_{\mathcal{A}} u_{t}\right|^{2}\right) \mathrm{d} y+\int \bar{\rho}\left|u_{t t}\right|^{2} \mathrm{~d} y \\
& =\int J\left(\nabla\left(P^{\prime}(\bar{\rho}) \operatorname{div}(\bar{\rho} u)\right)+g \operatorname{div}(\bar{\rho} u) e_{3}+\partial_{t} \mathcal{L}_{M}\right) \cdot u_{t t} \mathrm{~d} y+\int J \mathcal{N}_{t} \cdot u_{t t} \mathrm{~d} y \\
& +\int J N_{u}^{t, 1} \cdot u_{t t} \mathrm{~d} y+\mu_{1} \int J \nabla_{\mathcal{A}} u_{t}: \nabla_{\mathcal{A}_{t}} u_{t} \mathrm{~d} y+\frac{\mu_{1}}{2} \int J_{t}\left|\nabla_{\mathcal{A}} u_{t}\right|^{2} \mathrm{~d} y \\
& +\mu_{2} \int J \operatorname{div}_{\mathcal{A}} u_{t}: \operatorname{div}_{\mathcal{A}_{t}} u_{t} \mathrm{~d} y+\frac{\mu_{2}}{2} \int J_{t}\left|\operatorname{div}_{\mathcal{A}} u_{t}\right|^{2} \mathrm{~d} y=: \sum_{k=1}^{7} I_{k}^{L} .
\end{aligned}
$$

On the other hand, making use of (3.26), (3.19), (3.15), (3.14), (3.9) and (3.7), the seven integral terms $I_{1}^{L}-I_{7}^{L}$ can be bounded as follows.

$$
\begin{aligned}
& I_{1}^{L} \lesssim\|u\|_{2}\left\|u_{t t}\right\|_{0}, \quad I_{2}^{L} \lesssim\left\|\mathcal{N}_{t}\right\|_{0}\left\|u_{t t}\right\|_{0} \lesssim \sqrt{\mathcal{E}^{H}} \mathcal{D}^{L}, \quad I_{3}^{L} \lesssim\left\|N_{u}^{t, 1}\right\|_{0}\left\|u_{t t}\right\|_{0} \lesssim \sqrt{\mathcal{E}^{H}} \mathcal{D}^{L} \\
& I_{4}^{L} \lesssim\left\|\nabla_{\mathcal{A}} u_{t}\right\|_{0}\left\|\nabla_{\mathcal{A}_{t}} u_{t}\right\|_{0} \lesssim \sqrt{\mathcal{E}^{H}} \mathcal{D}^{L}, \quad I_{5}^{L} \lesssim\left\|J_{t}\right\|_{2}\left\|\nabla_{\mathcal{A}} u_{t}\right\|_{0}^{2} \lesssim \sqrt{\mathcal{E}^{H}} \mathcal{D}^{L} \\
& I_{6}^{L} \lesssim\left\|\operatorname{div}_{\mathcal{A}} u_{t}\right\|_{0}\left\|\operatorname{div}_{\mathcal{A}_{t}} u_{t}\right\|_{0} \lesssim \sqrt{\mathcal{E}^{H}} \mathcal{D}^{L}, \quad I_{7}^{L} \lesssim\left\|J_{t}\right\|_{2}\left\|\operatorname{div}_{\mathcal{A}} u_{t}\right\|_{0}^{2} \lesssim \sqrt{\mathcal{E}^{H}} \mathcal{D}^{L} .
\end{aligned}
$$

Thus, plugging the above seven estimates into (3.29) and applying Cauchy-Schwarz's inequality, one obtains Lemma 3.5 immediately.

\section{3. $y_{\mathrm{v}}$-derivative estimates}

In this subsection we establish the $y_{\mathrm{v}}$-derivative estimates. To this end, we rewrite (2.22) as the following non-homogeneous linear form:

$$
\left\{\begin{array}{l}
\eta_{t}=u, \\
\bar{\rho} J^{-1} u_{t}-\mu_{1} \Delta u-\mu_{2} \nabla \operatorname{div} u-\nabla\left(P^{\prime}(\bar{\rho}) \operatorname{div}(\bar{\rho} \eta)\right)=g \operatorname{div}(\bar{\rho} \eta) e_{3}+\mathcal{L}_{M}+\mathcal{N}+N_{u},
\end{array}\right.
$$

where

$$
N_{u}:=\mu_{1}\left(\operatorname{div}_{\tilde{\mathcal{A}}} \nabla_{\tilde{\mathcal{A}}} u+\operatorname{div} \nabla_{\tilde{\mathcal{A}}} u+\operatorname{div}_{\tilde{\mathcal{A}}} \nabla u\right)+\mu_{2}\left(\nabla_{\tilde{\mathcal{A}}} \operatorname{div}_{\tilde{\mathcal{A}}} u+\nabla \operatorname{div}_{\tilde{\mathcal{A}}} u+\nabla_{\tilde{\mathcal{A}}} \operatorname{div} u\right) .
$$

Moreover, we employ (3.16), (3.15), (3.5), and the interpolation inequality to control the term $N_{u}$ as follows.

$$
\begin{aligned}
& \left\|N_{u}\right\|_{3} \lesssim\|\eta\|_{3}\|u\|_{5}+\|\eta\|_{5}\|u\|_{3} \\
& \left\|N_{u}\right\|_{4} \lesssim\|\eta\|_{3}\|u\|_{6}+\|\eta\|_{6}\|u\|_{3}, \\
& \left\|N_{u}\right\|_{5} \lesssim \sqrt{\mathcal{E}^{L}}\|(\eta, u)\|_{7}+\|\eta\|_{6}\|u\|_{4} \lesssim \sqrt{\mathcal{E}^{L}}\|(\eta, u)\|_{7} \\
& \left\|\partial_{t} N_{u}\right\|_{0} \lesssim \sqrt{\mathcal{E}^{H} \mathcal{D}^{L}}, \quad\left\|\partial_{t}^{j} N_{u}\right\|_{4-2 j} \lesssim \sqrt{\mathcal{E}^{L} \mathcal{E}^{H}} \\
& \left\|\partial_{t}^{j} N_{u}\right\|_{5-2 j} \lesssim \sqrt{\mathcal{E}^{L} \mathcal{D}^{H}}+\|u\|_{4}^{2}+\|\eta\|_{5}\left\|u_{t}\right\|_{3} \lesssim \sqrt{\mathcal{E}^{L} \mathcal{D}^{H}}, \quad j=1,2 .
\end{aligned}
$$

Thus, we have the following bounds on $\eta$.

Lemma 3.6. It holds that

$$
\begin{aligned}
& \frac{\mathrm{d}}{\mathrm{d} t}\left(\int \bar{\rho} J^{-1} \partial_{\mathrm{v}}^{j} \eta \cdot \partial_{\mathrm{v}}^{j} u \mathrm{~d} y+\frac{1}{2}\left\|\partial_{\mathrm{v}}^{j}\left(\sqrt{\mu_{1}} \nabla \eta, \sqrt{\mu_{2}} \operatorname{div} \eta\right)\right\|_{0}^{2}\right)+c\left\|\partial_{\mathrm{v}}^{j}\left(\partial_{1} \eta, \operatorname{div} \eta\right)\right\|_{0}^{2} \\
& \quad \lesssim \operatorname{sign}(j)\left(\left\|\left(\partial_{1} \eta, \operatorname{div} \eta, u_{t}\right)\right\|_{\underline{j-1,0}}^{2}\right)+\left\|\partial_{\mathrm{v}}^{j} u\right\|_{0}^{2}+\sqrt{\mathcal{E}^{H}} \mathcal{D}^{L}, \quad j=0, \cdots, 3,
\end{aligned}
$$

where $\operatorname{sign}(j)=1$ when $j \neq 0$ and $=0$ when $j=0$. 
ProOf. Here we only prove the case $j=3$ and the rest three cases can be shown in the same manner. Applying $\partial_{\mathrm{v}}^{3}$ to $(\underline{3.30})_{2}$, multiplying the resulting equality by $\partial_{\mathrm{v}}^{3} \eta$, and then using $(\underline{3.30})_{1}$, we find that

$$
\begin{aligned}
& \bar{\rho} J^{-1} \partial_{t}\left(\partial_{\mathrm{v}}^{3} \eta \cdot \partial_{\mathrm{v}}^{3} u\right)-\left(\mu_{1} \Delta \partial_{\mathrm{v}}^{3} \eta_{t}+\mu_{2} \nabla \operatorname{div} \partial_{\mathrm{v}}^{3} \eta_{t}+\partial_{\mathrm{v}}^{3} \nabla\left(P^{\prime}(\bar{\rho}) \operatorname{div}(\bar{\rho} \eta)\right) \cdot \partial_{\mathrm{v}}^{3} \eta\right. \\
& =\partial_{\mathrm{v}}^{3}\left(g \operatorname{div}(\bar{\rho} \eta) e_{3}+\mathcal{L}_{M}+\mathcal{N}+N_{u}\right) \cdot \partial_{\mathrm{v}}^{3} \eta+\bar{\rho} J^{-1}\left|\partial_{\mathrm{v}}^{3} u\right|^{2}-\sum_{k=0}^{2}\left(\partial_{\mathrm{v}}^{3-k}\left(\bar{\rho} J^{-1}\right) \partial_{\mathrm{v}}^{k} u_{t}\right) \cdot \partial_{\mathrm{v}}^{3} \eta
\end{aligned}
$$

Integrating (by parts) the above identity over $\Omega$, we have

$$
\begin{aligned}
& \frac{\mathrm{d}}{\mathrm{d} t}\left(\int \bar{\rho} J^{-1} \partial_{\mathrm{v}}^{3} \eta \cdot \partial_{\mathrm{v}}^{3} u \mathrm{~d} y+\frac{\mu_{1}}{2} \int\left|\nabla \partial_{\mathrm{v}}^{3} \eta\right|^{2} \mathrm{~d} y+\frac{\mu_{2}}{2} \int_{\Omega}\left|\operatorname{div} \partial_{\mathrm{v}}^{3} \eta\right|^{2} \mathrm{~d} y\right) \\
& =\int \partial_{\mathrm{v}}^{3}(g \operatorname{div}(\bar{\rho} \eta)) \partial_{\mathrm{v}}^{3} \eta_{3} \mathrm{~d} y-\int \partial_{\mathrm{v}}^{3}\left(P^{\prime}(\bar{\rho}) \operatorname{div}(\bar{\rho} \eta)\right) \operatorname{div} \partial_{\mathrm{v}}^{3} \eta \mathrm{d} y+\int \partial_{\mathrm{v}}^{3} \mathcal{L}_{M} \cdot \partial_{\mathrm{v}}^{3} \eta \mathrm{d} y \\
& \quad+\int \partial_{\mathrm{v}}^{3} \mathcal{N} \cdot \partial_{\mathrm{v}}^{3} \eta \mathrm{d} y+\int \partial_{\mathrm{v}}^{3} N_{u} \cdot \partial_{\mathrm{v}}^{3} \eta \mathrm{d} y-\sum_{k=0}^{2} \int\left(\partial_{\mathrm{v}}^{3}\left(\bar{\rho} J^{-1} u_{t}\right)-\bar{\rho} J^{-1} \partial_{\mathrm{v}}^{3} u_{t}\right) \cdot \partial_{\mathrm{v}}^{3} \eta \mathrm{d} y \\
& \quad+\int \bar{\rho} J_{t}^{-1} \partial_{\mathrm{v}}^{3} \eta \cdot \partial_{\mathrm{v}}^{3} u \mathrm{~d} y+\int \bar{\rho} J^{-1}\left|\partial_{\mathrm{v}}^{3} u\right|^{2} \mathrm{~d} y \leq \sum_{k=1}^{7} J_{k}^{L}+c\left\|\partial_{\mathrm{v}}^{3} u\right\|_{0}^{2},
\end{aligned}
$$

where the first seven integrals on the right hand of (3.36) are denoted by $J_{1}^{L}-J_{7}^{L}$, respectively.

In view of (2.2) and (2.3), we see that $J_{1}^{L}$ can be bounded as follows.

$$
\begin{aligned}
J_{1}^{L}= & \int g \bar{\rho}^{\prime}\left|\partial_{\mathrm{v}}^{3} \eta_{3}\right|^{2} \mathrm{~d} y+\int g \bar{\rho} \partial_{\mathrm{v}}^{3} \eta_{3} \operatorname{div} \partial_{\mathrm{v}}^{3} \eta \mathrm{d} y \\
& +\sum_{k=0}^{2} \int \partial_{\mathrm{v}}^{3} \eta_{3}\left(\left(\partial_{\mathrm{v}}^{3}\left(g \bar{\rho}^{\prime} \eta_{3}\right)-g \bar{\rho}^{\prime} \partial_{\mathrm{v}}^{3} \eta_{3}\right)+\left(\partial_{\mathrm{v}}^{3}(g \bar{\rho} \operatorname{div} \eta)-g \bar{\rho} \operatorname{div} \partial_{\mathrm{v}}^{3} \eta\right)\right) \mathrm{d} y \\
\leq & \int g \bar{\rho}^{\prime}\left|\partial_{\mathrm{v}}^{3} \eta_{3}\right|^{2} \mathrm{~d} y+\int g \bar{\rho} \partial_{\mathrm{v}}^{3} \eta_{3} \operatorname{div} \partial_{\mathrm{v}}^{3} \eta \mathrm{d} y+c\left\|\partial_{\mathrm{v}}^{3} \eta_{3}\right\|\left\|_{0}\right\|(\eta, \operatorname{div} \eta) \|_{2}, 0 \\
J_{2}^{L}=- & \int P^{\prime}(\bar{\rho}) \bar{\rho}\left|\operatorname{div} \partial_{\mathrm{v}}^{3} \eta\right|^{2} \mathrm{~d} y-\int \bar{P}^{\prime} \partial_{\mathrm{v}}^{3} \eta_{3} \operatorname{div} \partial_{\mathrm{v}}^{3} \eta \mathrm{d} y \\
- & \sum_{k=0}^{2} \int\left(\partial_{\mathrm{v}}^{3}\left(P^{\prime}(\bar{\rho}) \bar{\rho} \operatorname{div} \eta\right)-P^{\prime}(\bar{\rho}) \bar{\rho} \operatorname{div} \partial_{\mathrm{v}}^{3} \eta+\partial_{\mathrm{v}}^{3}\left(\bar{P}^{\prime} \eta_{3}\right)-\bar{P}^{\prime} \partial_{\mathrm{v}}^{3} \eta_{3}\right) \operatorname{div} \partial_{\mathrm{v}}^{3} \eta \mathrm{d} y \\
\leq & -\int P^{\prime}(\bar{\rho}) \bar{\rho}\left|\operatorname{div} \partial_{\mathrm{v}}^{3} \eta\right|^{2} \mathrm{~d} y-\int \bar{P}^{\prime} \partial_{\mathrm{v}}^{3} \eta_{3} \operatorname{div} \partial_{\mathrm{v}}^{3} \eta \mathrm{d} y+c\left\|\operatorname{div} \partial_{\mathrm{v}}^{3} \eta\right\|_{0}\|(\eta, \operatorname{div} \eta)\|_{2,0}
\end{aligned}
$$

and

$$
\begin{aligned}
J_{3}^{L}= & \lambda \int\left(m^{2} \partial_{1}^{2} \partial_{\mathrm{v}}^{3} \eta-m^{2} \partial_{1} \operatorname{div} \partial_{\mathrm{v}}^{3} \eta e_{1}+\nabla\left(m m^{\prime} \partial_{\mathrm{v}}^{3} \eta_{3}+m^{2} \operatorname{div}_{\mathrm{v}} \partial_{\mathrm{v}}^{3} \eta_{\mathrm{v}}\right) \cdot \partial_{\mathrm{v}}^{3} \eta \mathrm{d} y\right. \\
& -\lambda \sum_{k=0}^{2} \int\left(\partial_{\mathrm{v}}^{3}\left(m^{2}\left(\partial_{1} \eta-\operatorname{div} \eta e_{1}\right)\right)-m^{2} \partial_{\mathrm{v}}^{3}\left(\partial_{1} \eta-\operatorname{div} \eta e_{1}\right)\right) \cdot \partial_{1} \partial_{\mathrm{v}}^{3} \eta \\
& \left.+\left(\partial_{\mathrm{v}}^{3}\left(m m^{\prime} \eta_{3}\right)-m m^{\prime} \partial_{\mathrm{v}}^{3} \eta_{3}+\partial_{\mathrm{v}}^{3}\left(m^{2} \operatorname{div}_{\mathrm{v}} \eta_{\mathrm{v}}\right)-m^{2} \operatorname{div}_{\mathrm{v}} \partial_{\mathrm{v}}^{3} \eta_{\mathrm{v}}\right) \operatorname{div} \partial_{\mathrm{v}}^{3} \eta\right) \mathrm{d} y \\
\leq & -\lambda \int m^{2}\left(\left|\partial_{1} \partial_{\mathrm{v}}^{3} \eta_{\mathrm{v}}\right|^{2}+\left|\operatorname{div}_{\mathrm{v}} \partial_{\mathrm{v}}^{3} \eta_{\mathrm{v}}\right|^{2}\right) \mathrm{d} y-\lambda \int m m^{\prime} \partial_{\mathrm{v}}^{3} \eta_{3} \operatorname{div}_{\mathrm{v}}^{3} \eta \mathrm{d} y \\
& +c\left\|\partial_{\mathrm{v}}^{3}\left(\partial_{1} \eta, \operatorname{div} \eta\right)\right\|_{0}\left\|\left(\eta, \partial_{1} \eta, \operatorname{div} \eta\right)\right\|_{2,0} .
\end{aligned}
$$


Inserting the above three estimates into (3.36), we deduce by (3.6) and (1.5) that

$$
\begin{aligned}
& \frac{\mathrm{d}}{\mathrm{d} t}\left(\int \bar{\rho} J^{-1} \partial_{\mathrm{v}}^{3} \eta \cdot \partial_{\mathrm{v}}^{3} u \mathrm{~d} y+\frac{1}{2}\left\|\partial_{\mathrm{v}}^{3}\left(\sqrt{\mu_{1}} \nabla \eta, \sqrt{\mu_{2}} \operatorname{div} \eta\right)\right\|_{0}^{2}\right)-E\left(\partial_{\mathrm{v}}^{3} \eta\right) \\
& \quad \leq c\left(\left\|\partial_{\mathrm{v}}^{3} u\right\|_{0}^{2}+\left\|\partial_{\mathrm{v}}^{3}\left(\partial_{1} \eta, \operatorname{div} \eta\right)\right\|_{0}\left\|\left(\partial_{1} \eta, \operatorname{div} \eta\right)\right\|_{2}, 0\right)+\sum_{k=4}^{7} J_{k}^{L} .
\end{aligned}
$$

On the other hand, by (3.31), (3.18), 3.12), (3.11) and (3.3), we have

$$
\begin{aligned}
& J_{4}^{L} \lesssim\left\|\partial_{\mathrm{v}}^{3} \mathcal{N}\right\|_{0}\left\|\partial_{\mathrm{v}}^{3} \eta\right\|_{0} \lesssim \sqrt{\mathcal{E}^{H}} \mathcal{D}^{L} \\
& J_{5}^{L} \lesssim\left\|\partial_{\mathrm{v}}^{3} N_{u}\right\|_{0}\left\|\partial_{\mathrm{v}}^{3} \eta\right\|_{0} \lesssim \sqrt{\mathcal{E}^{H}} \mathcal{D}^{L}, \\
& J_{6}^{L} \lesssim\left(\left\|J^{-1}-1\right\|_{5}+1\right)\left\|u_{t}\right\|_{2}, 0\left\|\partial_{\mathrm{v}}^{3} \eta\right\|_{0} \lesssim\left\|u_{t}\right\|_{2,0}\left\|\partial_{\mathrm{v}}^{3} \eta\right\|_{0}, \\
& J_{7}^{L} \lesssim\left\|J_{t}^{-1}\right\|_{2}\left\|\partial_{\mathrm{v}}^{3} \eta\right\|_{0}\left\|\partial_{\mathrm{v}}^{3} u\right\|_{0} \lesssim \sqrt{\mathcal{E}^{H}} \mathcal{D}^{L} \text {. }
\end{aligned}
$$

Consequently, putting the above four estimates into (3.40), and using Lemma [3.4, (3.6) and Cauchy-Schwarz's inequality, we deduce the desired conclusion for the case $j=3$.

Similarly, we can also establish the $y_{\mathrm{v}}$-derivative estimates on $u$.

Lemma 3.7. It holds that

$$
\frac{\mathrm{d}}{\mathrm{d} t}\left(\left\|\sqrt{\bar{\rho} J^{-1}} \partial_{\mathrm{v}}^{j} u\right\|_{0}^{2}-E\left(\partial_{\mathrm{v}}^{j} \eta\right)\right)+c\left\|\nabla \partial_{\mathrm{v}}^{j} u\right\|_{0}^{2} \lesssim \operatorname{sign}(j)\left\|\left(\partial_{1} \eta, \operatorname{div} \eta, u_{t}\right)\right\|_{j-1,0}^{2}+\sqrt{\mathcal{E}^{H}} \mathcal{D}^{L}
$$

for $j=0,1,2,3$.

ProOF. We only show the case $j=3$ and the rest cases can be proved in the same manner. We apply $\partial_{\mathrm{v}}^{3}$ to $(3.30)_{2}$ and multiply the resulting equation by $\partial_{\mathrm{v}}^{3} u$ to get

$$
\begin{aligned}
& \bar{\rho} J^{-1} \partial_{\mathrm{v}}^{3} u_{t} \cdot \partial_{\mathrm{v}}^{3} u-\left(\mu_{1} \Delta \partial_{\mathrm{v}}^{3} u+\mu_{2} \nabla \operatorname{div} \partial_{\mathrm{v}}^{3} u+\partial_{\mathrm{v}}^{3} \nabla\left(P^{\prime}(\bar{\rho}) \operatorname{div}(\bar{\rho} \eta)\right) \cdot \partial_{\mathrm{v}}^{3} u\right. \\
& =\partial_{\mathrm{v}}^{3}\left(g \operatorname{div}(\bar{\rho} \eta) e_{3}+\mathcal{L}_{M}+\mathcal{N}+N_{u}\right) \cdot \partial_{\mathrm{v}}^{3} u-\sum_{k=0}^{2} \partial_{\mathrm{v}}^{3-k}\left(\bar{\rho} J^{-1}\right) \partial_{\mathrm{v}}^{k} u_{t} \cdot \partial_{\mathrm{v}}^{3} u .
\end{aligned}
$$

Integrating (by parts) the above identity over $\Omega$, one has

$$
\begin{aligned}
& \frac{1}{2} \frac{\mathrm{d}}{\mathrm{d} t} \int \bar{\rho} J^{-1}\left|\partial_{\mathrm{v}}^{3} u\right|^{2} \mathrm{~d} y+\mu_{1} \int\left|\nabla \partial_{\mathrm{v}}^{3} u\right|^{2} \mathrm{~d} y+\mu_{2} \int\left|\operatorname{div} \partial_{\mathrm{v}}^{3} u\right|^{2} \mathrm{~d} y \\
& =\int \partial_{\mathrm{v}}^{3}(g \operatorname{div}(\bar{\rho} \eta)) \partial_{\mathrm{v}}^{3} u_{3} \mathrm{~d} y-\int \partial_{\mathrm{v}}^{3}\left(P^{\prime}(\bar{\rho}) \operatorname{div}(\bar{\rho} \eta)\right) \operatorname{div} \partial_{\mathrm{v}}^{3} u \mathrm{~d} y+\int \partial_{\mathrm{v}}^{3} \mathcal{L}_{M} \cdot \partial_{\mathrm{v}}^{3} u \mathrm{~d} y \\
& \quad+\int \partial_{\mathrm{v}}^{3} \mathcal{N} \cdot \partial_{\mathrm{v}}^{3} u \mathrm{~d} y+\int \partial_{\mathrm{v}}^{3} N_{u} \cdot \partial_{\mathrm{v}}^{3} u \mathrm{~d} y+\frac{1}{2} \int \bar{\rho} J_{t}^{-1}\left|\partial_{\mathrm{v}}^{3} u\right|^{2} \mathrm{~d} y \\
& \quad-\sum_{k=0}^{2} \int \partial_{\mathrm{v}}^{3-k}\left(\bar{\rho} J^{-1}\right) \partial_{\mathrm{v}}^{k} u_{t} \cdot \partial_{\mathrm{v}}^{3} u \mathrm{~d} y=: \sum_{k=1}^{7} K_{k}^{L} .
\end{aligned}
$$

Similarly to (3.37)-(3.39), the three integrals $K_{1}^{L}-K_{3}^{L}$ can be controlled as follows.

$$
\begin{aligned}
K_{1}^{L} \leq & \frac{1}{2} \frac{\mathrm{d}}{\mathrm{d} t}\left(\int g \bar{\rho}^{\prime}\left|\partial_{\mathrm{v}}^{3} \eta_{3}\right|^{2} \mathrm{~d} y+2 \int g \bar{\rho} \partial_{\mathrm{v}}^{3} \eta_{3} \operatorname{div} \partial_{\mathrm{v}}^{3} \eta \mathrm{d} y\right)-\int g \bar{\rho} \partial_{\mathrm{v}}^{3} \eta_{3} \operatorname{div} \partial_{\mathrm{v}}^{3} u \mathrm{~d} y \\
& +c\left\|\partial_{\mathrm{v}}^{3} u_{3}\right\|_{0}\|(\eta, \operatorname{div} \eta)\|_{2}, 0 \\
K_{2}^{L} \leq & -\frac{1}{2} \frac{\mathrm{d}}{\mathrm{d} t} \int P^{\prime}(\bar{\rho}) \bar{\rho}\left|\operatorname{div} \partial_{\mathrm{v}}^{3} \eta\right|^{2} \mathrm{~d} y-\int \bar{P}^{\prime} \partial_{\mathrm{v}}^{3} \eta_{3} \operatorname{div} \partial_{\mathrm{v}}^{3} u \mathrm{~d} y \\
& +c\left\|\operatorname{div} \partial_{\mathrm{v}}^{3} u\right\|_{0}\|(\eta, \operatorname{div} \eta)\|_{2}, 0
\end{aligned}
$$


and

$$
\begin{aligned}
K_{3}^{L} \leq & -\lambda \int\left(\left(m^{2} \partial_{1} \partial_{\mathrm{v}}^{3} \eta-m^{2} \operatorname{div} \partial_{\mathrm{v}}^{3} \eta e_{1}\right) \cdot \partial_{1} \partial_{\mathrm{v}}^{3} u\right. \\
& \left.+\left(m^{2} \operatorname{div} \partial_{\mathrm{v}}^{3} \eta+m m^{\prime} \partial_{\mathrm{v}}^{3} \eta_{3}-m^{2} \partial_{1} \partial_{\mathrm{v}}^{3} \eta_{1}\right) \operatorname{div} \partial_{\mathrm{v}}^{3} u\right) \mathrm{d} y \\
& +c\left\|\partial_{\mathrm{v}}^{3}\left(\partial_{1} u, \operatorname{div} u\right)\right\|_{0}\left\|\left(\eta, \partial_{1} \eta, \operatorname{div} \eta\right)\right\|_{2}, 0 \\
\leq & -\frac{\lambda}{2} \frac{\mathrm{d}}{\mathrm{d} t} \int m^{2}\left(\left|\partial_{1} \partial_{\mathrm{v}}^{3} \eta_{\mathrm{v}}\right|^{2}+\left|\operatorname{div}_{\mathrm{v}} \partial_{\mathrm{v}}^{3} \eta_{\mathrm{v}}\right|^{2}\right) \mathrm{d} y-\lambda \int m m^{\prime} \partial_{\mathrm{v}}^{3} \eta_{3} \operatorname{div} \partial_{\mathrm{v}}^{3} u \mathrm{~d} y \\
& +c\left\|\partial_{\mathrm{v}}^{3}\left(\partial_{1} u, \operatorname{div} u\right)\right\|_{0}\left\|\left(\eta, \partial_{1} \eta, \operatorname{div} \eta\right)\right\|_{2}, 0 .
\end{aligned}
$$

Substituting the above three estimates into (3.45), using (3.6) and (1.5), we conclude that

$$
\frac{1}{2} \frac{\mathrm{d}}{\mathrm{d} t}\left(\left\|\sqrt{\bar{\rho} J^{-1}} \partial_{\mathrm{v}}^{3} u\right\|_{0}^{2}-E\left(\partial_{\mathrm{v}}^{3} \eta\right)\right)+\left\|\partial_{\mathrm{v}}^{3}\left(\sqrt{\mu_{1}} \nabla u, \sqrt{\mu_{2}} \operatorname{div} u\right)\right\|_{0}^{2} \leq c\left\|\left(\partial_{1} \eta, \operatorname{div} \eta\right)\right\|_{\underline{2}, 0}^{2}+\sum_{k=4}^{7} K_{k}^{L}
$$

On the other hand, similarly to (3.41)-(3.44), one obtains

$$
\sum_{k=4}^{7} K_{k}^{L} \lesssim\left\|u_{t}\right\|_{2,0}\left\|\partial_{\mathrm{v}}^{3} u\right\|_{0}+\sqrt{\mathcal{E}^{H}} \mathcal{D}^{L}
$$

Consequently, inserting the above inequality into (3.49), and using (3.6) and Cauchy-Schwarz's inequality, we obtain Lemma 3.7.

\section{4. y-derivative estimates}

In this subsection we derive the $y$-derivative estimates of $\eta$ and $u$. Similarly to the incompressible magnetic RT problem see [28, Lemma 2.4]), we focus on the term $\partial_{1}^{2} \eta$ and extract the following equations from the second and third components of $(3.30)_{2}$ :

$$
\begin{aligned}
& -\mu_{1} \partial_{1}^{2} \partial_{t} \eta_{\mathrm{v}}-\lambda m^{2} \partial_{1}^{2} \eta_{\mathrm{v}}=\mu_{1} \Delta_{\mathrm{v}} u_{\mathrm{v}}+\mu_{2} \nabla_{\mathrm{v}} \operatorname{div} u \\
& +\nabla_{\mathrm{v}}\left(P^{\prime}(\bar{\rho}) \operatorname{div}(\bar{\rho} \eta)+\lambda m m^{\prime} \eta_{3}+\lambda m^{2} \operatorname{div}_{\mathrm{v}} \eta_{\mathrm{v}}\right)+g \operatorname{div}(\bar{\rho} \eta)(0,1)^{\mathrm{T}}-\bar{\rho} J^{-1} \partial_{t} u_{\mathrm{v}}+N_{\mathrm{v}}+N_{u, \mathrm{v}}
\end{aligned}
$$

Meanwhile, the first component of $(3.30)_{2}$ can be written as

$$
\begin{aligned}
& -\mu \partial_{1} \operatorname{div} \eta_{t}-P^{\prime}(\bar{\rho}) \bar{\rho} \partial_{1} \operatorname{div} \eta \\
& \quad=\mu_{1} \Delta_{\mathrm{v}} u_{1}+\partial_{1}\left(\bar{P}^{\prime} \eta_{3}+\lambda m m^{\prime} \eta_{3}-\mu_{1} \operatorname{div}_{\mathrm{v}} u_{\mathrm{v}}\right)-\bar{\rho} J^{-1} \partial_{t} u_{1}+N_{1}+N_{u, 1},
\end{aligned}
$$

where $\mu:=\mu_{1}+\mu_{2}, \mathcal{N}:=\left(N_{1}, N_{\mathrm{v}}\right)$ and $N_{u}:=\left(N_{u, 1}, N_{u, \mathrm{v}}\right)$.

Noting that the order of $\partial_{1}$ in the linear part on the right hand side of (3.50) is lower than that on the left hand side, this feature provides a possibility that the $y_{1}$-derivative estimates of $\eta_{\mathrm{v}}$ can be converted to the $y_{\mathrm{v}}$-derivative estimates of $\eta$. Similarly, (3.51) also provides a possibility that the $y_{1}$-derivative estimates of $\eta_{1}$ can be converted to the the $y_{\mathrm{v}}$-derivative estimates of $\eta$ by the relation $\operatorname{div} \eta=\partial_{1} \eta_{1}+\operatorname{div}_{\mathrm{v}} \eta_{\mathrm{v}}$. Based on these basic observations, we can establish the following $y$-derivative estimates on $\eta$.

Lemma 3.8. We have

$$
\frac{\mathrm{d}}{\mathrm{d} t} \mathcal{H}_{2}(\eta)+\left\|\left(\partial_{1} \eta, \operatorname{div} \eta\right)\right\|_{3}^{2}+\|u\|_{4}^{2} \lesssim\left\|\left(\partial_{1} \eta, \operatorname{div} \eta, \nabla u\right)\right\|_{\underline{3}, 0}^{2}+\left\|u_{t}\right\|_{2}^{2}+\mathcal{E}^{H} \mathcal{D}^{L}
$$

where the energy functional $\mathcal{H}_{2}(\eta)$ satisfies

$$
\left\|\partial_{1}^{2} \eta\right\|_{2}^{2}-h_{2}\left\|\partial_{1} \eta_{\mathrm{v}}\right\|_{\underline{3}, 0}^{2} \lesssim \mathcal{H}_{2}(\eta)
$$

for some positive constant $h_{2}$, depending on $\Omega$ and other physical parameters. 
Proof. Applying $\|\cdot\|_{j, i-j}^{2}$ to (3.51), using (3.8), (3.5) and Cauchy-Schwarz's inequality, we deduce that for $0 \leq i \leq 5$,

$$
\begin{aligned}
& \frac{\mathrm{d}}{\mathrm{d} t}\left\|\sqrt{\mu P^{\prime}(\bar{\rho}) \bar{\rho}} \diamond \partial_{1} \operatorname{div} \eta\right\|_{j, i-j}^{2}+\left(\left\|\left(P^{\prime}(\bar{\rho}) \bar{\rho}\right) \diamond \partial_{1} \operatorname{div} \eta\right\|_{j, i-j}^{2}+\left\|\mu \partial_{1} \operatorname{div} u\right\|_{j, i-j}^{2}\right) \\
& \lesssim\left\|\mu_{1} \Delta_{\mathrm{v}} u_{1}+\partial_{1}\left(\bar{P}^{\prime} \eta_{3}+\lambda m m^{\prime} \eta_{3}-\mu_{1} \operatorname{div}_{\mathrm{v}} u_{\mathrm{v}}\right)-\bar{\rho} J^{-1} \partial_{t} u_{1}+N_{1}+N_{u, 1}\right\|_{j, i-j}^{2}+\left\|\partial_{1} \eta\right\|_{j, i-j}^{2} \\
& \lesssim\left\|\partial_{1} \eta\right\|_{\underline{j}, i-j}^{2}+\|u\|_{j+1, i-j+1}^{2}+\left\|\left(u_{t}, \mathcal{N}, N_{u}\right)\right\|_{i}^{2},
\end{aligned}
$$

which, together with (3.23) and (1.3), gives

$$
\begin{aligned}
& \frac{\mathrm{d}}{\mathrm{d} t}\left\|\sqrt{\mu P^{\prime}(\bar{\rho}) \bar{\rho}} \diamond \partial_{1} \operatorname{div} \eta\right\|_{j, i-j}^{2}+c\left(\left\|\partial_{1}^{2}\left(\eta_{1}, u_{1}\right)\right\|_{j, i-j}^{2}+\left\|\partial_{1}(\operatorname{div} \eta, \operatorname{div} u)\right\|_{j, i-j}^{2}\right) \\
& \lesssim\left\|\partial_{1} \eta\right\|_{\underline{j+1, i-j}}^{2}+\|u\|_{j+1, i-j+1}^{2}+\left\|\left(u_{t}, \mathcal{N}, N_{u}\right)\right\|_{i}^{2} .
\end{aligned}
$$

Similarly, one easily deduces from (3.50) that

$$
\begin{aligned}
& \frac{\mathrm{d}}{\mathrm{d} t}\left\|\sqrt{\mu_{1} \lambda} m \partial_{1}^{2} \eta_{\mathrm{v}}\right\|_{j, i-j}^{2}+c\left\|\partial_{1}^{2}\left(\eta_{\mathrm{v}}, u_{\mathrm{v}}\right)\right\|_{j, i-j}^{2} \\
& \lesssim \| \mu_{1} \Delta_{\mathrm{v}} u_{\mathrm{v}}+\mu_{2} \nabla_{\mathrm{v}} \operatorname{div} u+\nabla_{\mathrm{v}}\left(P^{\prime}(\bar{\rho}) \operatorname{div}(\bar{\rho} \eta)+\lambda m m^{\prime} \eta_{3}+\lambda m^{2} \operatorname{div}_{\mathrm{v}} \eta_{\mathrm{v}}\right) \\
& +g \operatorname{div}(\bar{\rho} \eta)(0,1)^{\mathrm{T}}-\bar{\rho} J^{-1} \partial_{t} u_{\mathrm{v}}+N_{\mathrm{v}}+N_{u, \mathrm{v}} \|_{j, i-j}^{2} \\
& \lesssim\left\|\left(\eta, \operatorname{div}_{\mathrm{v}} \eta_{\mathrm{v}}, \operatorname{div} \eta\right)\right\|_{\underline{j+1, i-j}}^{2}+\|u\|_{j+1, i-j+1}^{2}+\left\|\left(u_{t}, \mathcal{N}, N_{u}\right)\right\|_{i}^{2} .
\end{aligned}
$$

Thus, using (3.23) and (3.6), we infer from (3.53) and (3.54) that

$$
\begin{aligned}
& \left.\frac{\mathrm{d}}{\mathrm{d} t}\left(\left\|\sqrt{\mu_{1} \lambda} m \partial_{1}^{2} \eta_{\mathrm{v}}\right\|_{j, i-j}^{2}+\| \sqrt{\mu P^{\prime}(\bar{\rho}) \bar{\rho}} \diamond \partial_{1} \operatorname{div} \eta\right) \|_{j, i-j}^{2}\right) \\
& \quad+c\left(\left\|\left(\partial_{1} \eta, \operatorname{div} \eta\right)\right\|_{j, i-j+1}^{2}+\|u\|_{j, i-j+2}^{2}\right) \lesssim\left\|\left(\partial_{1} \eta, \operatorname{div} \eta, \nabla u\right)\right\|_{j+1, i-j}^{2}+\left\|\left(u_{t}, \mathcal{N}, N_{u}\right)\right\|_{i}^{2}
\end{aligned}
$$

Adding up (3.55) from 0 to $j$, one gets

$$
\begin{aligned}
& \frac{\mathrm{d}}{\mathrm{d} t}\left(\left\|\sqrt{\mu_{1} \lambda} m \partial_{1}^{2} \eta_{\mathrm{v}}\right\|_{\underline{j}, i-j}^{2}+\left\|\sqrt{\mu P^{\prime}(\bar{\rho}) \bar{\rho}} \diamond \partial_{1} \operatorname{div} \eta\right\|_{\underline{j}, i-j}^{2}\right) \\
& \quad+c\left(\left\|\left(\partial_{1} \eta, \operatorname{div} \eta\right)\right\|_{\underline{j}, i-j+1}^{2}+\|u\|_{\underline{j}, i-j+2}^{2}\right) \lesssim\left\|\left(\partial_{1} \eta, \operatorname{div} \eta, \nabla u\right)\right\|_{\underline{j+1, i-j}}^{2}+\left\|\left(u_{t}, \mathcal{N}, N_{u}\right)\right\|_{i}^{2}
\end{aligned}
$$

In addition, we can use the relation (3.23) and the estimate

$$
\left\|\partial_{1} \eta_{\mathrm{v}}\right\|_{j+1, i-j}^{2} \lesssim\left\|\partial_{1}^{2} \eta_{\mathrm{v}}\right\|_{j+1, i-j-1}^{2}+\left\|\partial_{1} \eta_{\mathrm{v}}\right\|_{\underline{i+1,0}}^{2} \quad \text { for } 0 \leq j<i
$$

to find that

$$
\begin{aligned}
& \left\|\sqrt{\mu_{1} \lambda} m \partial_{1}^{2} \eta_{\mathrm{v}}\right\|_{\underline{i}, 0}^{2}+\left\|\sqrt{\mu P^{\prime}(\bar{\rho}) \bar{\rho}} \diamond \partial_{1} \operatorname{div} \eta\right\|_{\underline{i}, 0}^{2} \\
& \gtrsim\left\|\left(\partial_{1}^{2} \eta_{\mathrm{v}}, \partial_{1} \operatorname{div} \eta\right)\right\|_{i, 0}^{2} \gtrsim\left\|\partial_{1}^{2} \eta\right\|_{i, 0}^{2}-\tilde{h}_{i, i}\left\|\partial_{1} \eta_{\mathrm{v}}\right\|_{i+1,0}^{2}
\end{aligned}
$$

and

$$
\begin{aligned}
& \left.\left\|\sqrt{\mu_{1} \lambda} m \partial_{1}^{2} \eta_{\mathrm{v}}\right\|_{\underline{j}, i-j}^{2}+\| \sqrt{\mu P^{\prime}(\bar{\rho}) \bar{\rho}} \diamond \partial_{1} \operatorname{div} \eta\right)\left\|_{\underline{j}, i-j}^{2} \gtrsim\right\|\left(\partial_{1}^{2} \eta_{\mathrm{v}}, \partial_{1} \operatorname{div} \eta \|_{j, i-j}^{2}\right. \\
& \gtrsim\left\|\partial_{1}^{2} \eta\right\|_{j, i-j}^{2}-\tilde{h}_{i, j}\left(\left\|\partial_{1}^{2} \eta\right\|_{j+1, i-j-1}^{2}+\left\|\partial_{1} \eta_{\mathrm{v}}\right\|_{\underline{i+1,0}, 2}^{2}\right), \quad 0 \leq j<i
\end{aligned}
$$

for some positive constants $\tilde{h}_{i, j}$. Thus, we can further deduce from (3.56)-(3.58) that

$$
\frac{\mathrm{d}}{\mathrm{d} t} \mathcal{H}_{i}(\eta)+\left\|\left(\partial_{1} \eta, \operatorname{div} \eta\right)\right\|_{i+1}^{2}+\|u\|_{i+2}^{2} \lesssim\left\|\left(\partial_{1} \eta, \operatorname{div} \eta, \nabla u\right)\right\|_{\underline{i+1,0}}^{2}+\left\|\left(u_{t}, \mathcal{N}, N_{u}\right)\right\|_{i}^{2}
$$


where $\mathcal{H}_{i}(\eta):=\sum_{j=0}^{i} h_{i, j}\left(\left\|\sqrt{\mu_{1} \lambda} m \partial_{1}^{2} \eta_{\mathrm{v}}\right\|_{\underline{j}, i-j}^{2}+\left\|\sqrt{\mu P^{\prime}(\bar{\rho}) \bar{\rho}} \diamond \partial_{1} \operatorname{div} \eta\right\|_{\underline{j}, i-j}^{2}\right)$ satisfies

$$
\left\|\partial_{1}^{2} \eta\right\|_{i}^{2}-h_{i}\left\|\partial_{1} \eta_{\mathrm{v}}\right\|_{\underline{i+1}, 0}^{2} \lesssim \mathcal{H}_{i}(\eta)
$$

for some positive constants $h_{i, j}$ and $h_{i}$.

If we take $i=2$ in (3.59), we get

$$
\frac{\mathrm{d}}{\mathrm{d} t} \mathcal{H}_{2}(\eta)+\left\|\left(\partial_{1} \eta, \operatorname{div} \eta\right)\right\|_{3}^{2}+\|u\|_{4}^{2} \lesssim\left\|\left(\partial_{1} \eta, \operatorname{div} \eta, \nabla u\right)\right\|_{\underline{3}, 0}^{2}+\left\|u_{t}\right\|_{2}^{2}+\|\mathcal{N}\|_{2}^{2}+\left\|N_{u}\right\|_{2}^{2}
$$

On the other hand, by virtue of (3.31) and (3.18),

$$
\|\mathcal{N}\|_{2}^{2}+\left\|N_{u}\right\|_{2}^{2} \lesssim \mathcal{E}^{H} \mathcal{D}^{L}
$$

which combined with (3.60) gives the lemma.

Now, we turn to the derivation of $y$-derivative estimates of $u$. If we apply $\partial_{t}^{j}$ to $(\underline{3.30})_{2}$, we obtain

$$
\left\{\begin{array}{l}
-\mu_{1} \Delta \partial_{t}^{j} u-\mu_{2} \nabla \operatorname{div} \partial_{t}^{j} u=f_{j} \\
\left.\partial_{t}^{j} u\right|_{\partial \Omega}=0
\end{array}\right.
$$

where

$$
\begin{aligned}
f_{j}:= & \partial_{t}^{j}\left(\nabla\left(P^{\prime}(\bar{\rho}) \operatorname{div}(\bar{\rho} \eta)\right)+g \operatorname{div}(\bar{\rho} \eta) e_{3}+\mathcal{L}_{M}+\mathcal{N}+N_{u}\right) \\
& -\bar{\rho} J^{-1} \partial_{t}^{j+1} u-\bar{\rho} \sum_{k=0}^{j-1} C_{j}^{k} \partial_{t}^{j-k} J^{-1} \partial_{t}^{k+1} u
\end{aligned}
$$

Hence, one can apply the classical elliptic regularity theory to the above Lamé system [7, 31] to get

$$
\begin{aligned}
\left\|\partial_{t}^{j} u\right\|_{i-2 j+2}^{2} & \lesssim\left\|f_{j}\right\|_{i-2 j} \\
& \lesssim\left\|\partial_{t}^{j}\left(\nabla\left(P^{\prime}(\bar{\rho}) \operatorname{div}(\bar{\rho} \eta)\right)+g \operatorname{div}(\bar{\rho} \eta) e_{3}+\mathcal{L}_{M}\right)-\bar{\rho} J^{-1} \partial_{t}^{j+1} u\right\|_{i-2 j}^{2}+S_{j, i}
\end{aligned}
$$

where $S_{0, i}:=\left\|\left(\mathcal{N}, N_{u}\right)\right\|_{i}^{2}$ and

$$
S_{j, i}:=\left\|\partial_{t}^{j}\left(\mathcal{N}, N_{u}\right)\right\|_{i-2 j}^{2}+\left\|\bar{\rho} \sum_{k=0}^{j-1} \partial_{t}^{j-k} J^{-1} \partial_{t}^{k+1} u\right\|_{i-2 j}^{2} \text { for } j \geq 1 .
$$

Lemma 3.9. We have

$$
\begin{aligned}
& \|u\|_{3}^{2} \lesssim\|\eta\|_{3}^{2}+\left\|u_{t}\right\|_{1}^{2}+\mathcal{E}^{H} \mathcal{E}^{L} \\
& \left\|u_{t}\right\|_{2}^{2} \lesssim\|u\|_{2}^{2}+\left\|u_{t t}\right\|_{0}^{2}+\mathcal{E}^{H} \mathcal{D}^{L} .
\end{aligned}
$$

Proof. In view of (3.61) with $(i, j)=(1,0)$, one has

$$
\|u\|_{3}^{2} \lesssim\|\eta\|_{3}^{2}+\left\|u_{t}\right\|_{1}^{2}+S_{0,1}
$$

On the other hand, it follows from (3.31) and (3.18) that

$$
S_{0,1}=\left\|\left(\mathcal{N}, N_{u}\right)\right\|_{1}^{2} \lesssim \mathcal{E}^{H} \mathcal{E}^{L} .
$$

Therefore, we obtain (3.62) from the above two estimates.

To show (3.63), we take $(i, j)=(2,1)$ in (3.61) to find that

$$
\left\|u_{t}\right\|_{2}^{2} \lesssim\|u\|_{2}^{2}+\left\|u_{t t}\right\|_{0}^{2}+S_{1,2}
$$

Utilizing (3.34), (3.19), (3.12) and (3.5), we have

$$
S_{1,2}=\left\|\partial_{t}\left(\mathcal{N}, N_{u}\right)\right\|_{0}^{2}+\left\|\bar{\rho} J_{t}^{-1} u_{t}\right\|_{0}^{2} \lesssim \mathcal{E}^{H} \mathcal{D}^{L}
$$

The above two estimates give then (3.63). The proof is complete. 


\subsection{Lower-order energy inequality}

Now we are ready to build the lower-order energy inequality. In what follows the letters $c_{i}^{L}$, $i=1, \ldots, 9$, will denote generic positive constants which may depend on $\Omega$ and the physical parameters in the perturbation equations.

Proposition 3.1. Under the assumption (3.1), if $\delta$ is sufficiently small, then there is an energy functional $\tilde{\mathcal{E}}^{L}$ that is equivalent to $\mathcal{E}^{L}$, such that

$$
\frac{\mathrm{d}}{\mathrm{d} t} \tilde{\mathcal{E}}^{L}+\mathcal{D}^{L} \leq 0 \text { on }(0, T] .
$$

Proof. Noting that (3.4) still holds in the two-dimensional case, we have

$$
\left\|\left(\partial_{1} \eta, \operatorname{div} \eta\right)\right\|_{2,0}^{2} \leq \epsilon\left\|\left(\partial_{1} \eta, \operatorname{div} \eta\right)\right\|_{3,0}^{2}+C_{\epsilon}\left\|\left(\partial_{1} \eta, \operatorname{div} \eta\right)\right\|_{0}^{2} .
$$

Making use of the above interpolation inequality, Lemmas 3.6 3.7 and (3.6), we see that there is a constant $\kappa_{0,1}^{L} \geq 1$, such that

$$
\frac{\mathrm{d}}{\mathrm{d} t} \tilde{\mathcal{E}}_{1}^{L}+c_{1}^{L}\left\|\left(\partial_{1} \eta, \operatorname{div} \eta, \nabla u\right)\right\|_{\underline{3}, 0}^{2} \leq c_{2}^{L}\left(\left\|\left(\partial_{1} \eta, \operatorname{div} \eta\right)\right\|_{0}^{2}+\left\|u_{t}\right\|_{2}^{2}+\sqrt{\mathcal{E}^{H}} \mathcal{D}^{L}\right)
$$

holds for any $\kappa_{1}^{L} \geq \kappa_{0,1}^{L}$, where

$$
\begin{aligned}
\tilde{\mathcal{E}}_{1}^{L}:= & \sum_{\alpha_{1}+\alpha_{2} \leq 3}\left(\int \bar{\rho} J^{-1} \partial_{2}^{\alpha_{1}} \partial_{3}^{\alpha_{2}} \eta \cdot \partial_{2}^{\alpha_{1}} \partial_{3}^{\alpha_{2}} u \mathrm{~d} y-\kappa_{1}^{L} E\left(\partial_{2}^{\alpha_{1}} \partial_{3}^{\alpha_{2}} \eta\right)+\kappa_{1}^{L}\left\|\sqrt{\bar{\rho} J^{-1}} \partial_{2}^{\alpha_{1}} \partial_{3}^{\alpha_{2}} u\right\|_{0}^{2}\right) \\
& +\frac{1}{2}\left\|\left(\sqrt{\mu_{1}} \nabla \eta, \sqrt{\mu_{2}} \operatorname{div} \eta\right)\right\|_{\underline{3}, 0}^{2},
\end{aligned}
$$

and $\kappa_{0,1}^{L}$ depends on $\Omega$ and the physical parameters.

Putting the estimate (3.64) and Lemma 3.8 together, we arrive at

$$
\left.\frac{\mathrm{d}}{\mathrm{d} t}\left(\mathcal{H}_{2}(\eta)\right)+c_{3}^{L} \tilde{\mathcal{E}}_{1}^{L}\right)+\left\|\left(\partial_{1} \eta, \operatorname{div} \eta\right)\right\|_{3}^{2}+\|u\|_{4}^{2} \leq c_{4}^{L}\left(\left\|\left(\partial_{1} \eta, \operatorname{div} \eta\right)\right\|_{0}^{2}+\left\|u_{t}\right\|_{2}^{2}+\sqrt{\mathcal{E}^{H}} \mathcal{D}^{L}\right),
$$

which, together with (3.63), Lemma 3.5 and the interpolation inequality, yields

$$
\begin{aligned}
& \frac{\mathrm{d}}{\mathrm{d} t}\left(\mathcal{H}_{2}(\eta)+c_{3}^{L} \tilde{\mathcal{E}}_{1}^{L}+c_{5}^{L}\left\|\left(\sqrt{\mu_{1}} \nabla_{\mathcal{A}} u_{t}, \sqrt{\mu_{2}} \operatorname{div}_{\mathcal{A}} u_{t}\right)\right\|_{0}^{2}\right)+c_{6}^{L}\left(\left\|\left(\partial_{1} \eta, \operatorname{div} \eta\right)\right\|_{3}^{2}+\|u\|_{4}^{2}+\left\|u_{t t}\right\|_{0}^{2}\right) \\
& \quad \leq c_{7}^{L}\left(\left\|\left(\partial_{1} \eta, \operatorname{div} \eta, u\right)\right\|_{0}^{2}+\sqrt{\mathcal{E}^{H}} \mathcal{D}^{L}\right) .
\end{aligned}
$$

So, for a given $\kappa_{1}^{L}$, we can further deduce from (3.65) and Lemmas 3.6 3.7 with $j=0$ that there is a constant $\kappa_{0,2}^{L}>1$, such that

$$
\frac{\mathrm{d}}{\mathrm{d} t} \tilde{\mathcal{E}}_{2}^{L}+c_{7}^{L} \tilde{\mathcal{D}}^{L} \leq c_{8}^{L} \sqrt{\mathcal{E}^{H}} \mathcal{D}^{L} \quad \text { for any } \kappa_{2}^{L}>\kappa_{0,2}^{L},
$$

where

$$
\begin{aligned}
\tilde{\mathcal{E}}_{2}^{L}:= & \mathcal{H}_{2}(\eta)+c_{3}^{L} \tilde{\mathcal{E}}_{1}^{L}+c_{5}^{L}\left\|\left(\sqrt{\mu_{1} J} \nabla_{\mathcal{A}} u_{t}, \sqrt{\mu_{2} J} \operatorname{div}_{\mathcal{A}} u_{t}\right)\right\|_{0}^{2} \cdot+\left(\kappa_{2}^{L}\right)^{2}\left(\left\|\sqrt{\bar{\rho} J^{-1}} u\right\|_{0}^{2}-E(\eta)\right) \\
& +\kappa_{2}^{L}\left(\int \bar{\rho} J^{-1} \eta \cdot u \mathrm{~d} y+\frac{1}{2}\left\|\left(\sqrt{\mu_{1}} \nabla \eta, \sqrt{\mu_{2}} \operatorname{div} \eta\right)\right\|_{0}^{2}\right), \\
\tilde{\mathcal{D}}^{L}:= & \left\|\left(\partial_{1} \eta, \operatorname{div} \eta\right)\right\|_{3}^{2}+\|u\|_{4}^{2}+\left\|u_{t t}\right\|_{0}^{2},
\end{aligned}
$$


and $\kappa_{0,2}^{L}$ depends on $\kappa_{1}^{L}, \Omega$ and the physical parameters.

Obviously, to prove the proposition, it suffices to show

$$
\tilde{\mathcal{E}}_{2}^{L} \text { is equivalent to } \mathcal{E}^{L} \text { for some } \kappa_{1}^{L} \text { and } \kappa_{2}^{L}
$$

and

$$
\mathcal{D}^{L} \leq c_{9}^{L} \tilde{\mathcal{D}}^{L}
$$

We next verify these two facts.

Thanks to (3.16), (3.11), (3.7) and (3.6), it is easy to see that for sufficiently small $\delta$,

$$
\begin{aligned}
& \|f\|_{1}^{2} \lesssim\left\|\sqrt{J} \nabla_{\mathcal{A}} f\right\|_{0}^{2}+\left\|\sqrt{J} \operatorname{div}_{\mathcal{A}} f\right\|_{0}^{2} \lesssim\|f\|_{1}^{2}, \\
& \|f\|_{0}^{2} \lesssim\left\|\sqrt{\bar{\rho} J^{-1}} f\right\|_{0}^{2} \lesssim\|f\|_{0}^{2} .
\end{aligned}
$$

Moreover, $\|\eta\|_{4}$ is equivalent to the norm $\|\eta\|_{4,0}+\left\|\partial_{1} \eta\right\|_{3,0}+\left\|\partial_{1}^{2} \eta\right\|_{2}$. Thus, making use of CauchySchwarz's inequality, (3.52), Lemma 3.4, and (3.6), we conclude

$$
\|\eta\|_{4}^{2}+\left\|u_{t}\right\|_{1}^{2} \lesssim \tilde{\mathcal{E}}_{2}^{L}
$$

by choosing sufficiently large constants $\kappa_{1}^{L}$ and $\kappa_{2}^{L}$. Combining (3.70) with (3.62), one arrives at

$$
\mathcal{E}^{L} \lesssim \tilde{\mathcal{E}}_{2}^{L}+\mathcal{E}^{H} \mathcal{E}^{L}
$$

In particular, $\mathcal{E}^{L} \lesssim \tilde{\mathcal{E}}_{2}^{L}$ for sufficiently small $\delta$. On the other hand, by Cauchy-Schwarz's inequality we observe that $\tilde{\mathcal{E}}_{2}^{L} \lesssim \mathcal{E}^{L}$. This gives (3.67).

Finally, from (3.63) we get $\mathcal{D}^{L} \lesssim \tilde{\mathcal{D}}^{L}+\mathcal{E}^{H} \mathcal{D}^{L}$, which implies (3.68) for sufficiently small $\delta$. With (3.67) and (3.68) in hands, we immediately obtain the desired conclusion from (3.66) by defining $\tilde{\mathcal{E}}^{L}:=2 c_{9}^{L} \tilde{\mathcal{E}}_{2}^{L} / c_{7}^{L}$ and choosing $\delta \leq c_{7}^{L} / 2 c_{8}^{L} c_{9}^{L}$.

\section{Higher-order energy inequalities}

In this section we derive the higher-order energy inequalities for the transformed Parker problem. We shall first establish the higher-order versions of Lemmas 3.53 .9 in sequence.

Lemma 4.1. It holds that

$$
\frac{\mathrm{d}}{\mathrm{d} t}\left(\left\|\sqrt{\bar{\rho}} \partial_{t}^{3} u\right\|_{0}^{2}-E\left(u_{t t}\right)\right)+c\left\|\partial_{t}^{3} u\right\|_{1}^{2} \lesssim \sqrt{\mathcal{E}^{L}} \mathcal{D}^{H}
$$

Proof. Multiplying (3.25) $)_{2}$ with $j=3$ by $J \partial_{t}^{3} u$, integrating then (by parts) the resulting equations over $\Omega$, and using (2.22) $)_{1}$ and (2.7), we get

$$
\begin{aligned}
& \frac{1}{2} \frac{\mathrm{d}}{\mathrm{d} t} \int \bar{\rho}\left|\partial_{t}^{3} u\right|^{2} \mathrm{~d} y+\int J\left(\mu_{1}\left|\nabla_{\mathcal{A}} \partial_{t}^{3} u\right|^{2}+\mu_{2}\left|\operatorname{div}_{\mathcal{A}} \partial_{t}^{3} u\right|^{2}\right) \mathrm{d} y \\
& =\int\left(g \operatorname{div}\left(\bar{\rho} u_{t t}\right) \partial_{t}^{3} u_{3}-P^{\prime}(\bar{\rho}) \operatorname{div}\left(\bar{\rho} u_{t t}\right) \operatorname{div} \partial_{t}^{3} u\right) \mathrm{d} y+\int \partial_{t}^{3} \mathcal{L}_{M} \cdot \partial_{t}^{3} u \mathrm{~d} y \\
& \quad+\int J \partial_{t}^{3} \mathcal{N} \cdot \partial_{t}^{3} u \mathrm{~d} y+\int J N_{u}^{t, 3} \cdot \partial_{t}^{3} u \mathrm{~d} y \\
& \quad+\int(J-1)\left(g \operatorname{div}\left(\bar{\rho} u_{t t}\right) e_{3}+\nabla\left(P^{\prime}(\bar{\rho}) \operatorname{div}\left(\bar{\rho} u_{t t}\right)\right)+\partial_{t}^{3} \mathcal{L}_{M}\right) \cdot \partial_{t}^{3} u \mathrm{~d} y=: \sum_{k=1}^{5} I_{k}^{H},
\end{aligned}
$$


where the first two integrals on the right hand side can be written as

$$
\begin{aligned}
I_{1}^{H}= & \frac{1}{2} \frac{\mathrm{d}}{\mathrm{d} t}\left(\int g \bar{\rho}^{\prime}\left|\partial_{t}^{2} u_{3}\right|^{2} \mathrm{~d} y+2 \int g \bar{\rho} \partial_{t}^{2} u_{3} \operatorname{div} u_{t t} \mathrm{~d} y-\int P^{\prime}(\bar{\rho}) \bar{\rho}\left|\operatorname{div} u_{t t}\right|^{2} \mathrm{~d} y\right) \\
& -\int\left(\bar{P}^{\prime}+g \bar{\rho}\right) \partial_{t}^{2} u_{3} \operatorname{div} \partial_{t}^{3} u \mathrm{~d} y
\end{aligned}
$$

and

$$
I_{2}^{H}=-\frac{\lambda}{2} \frac{\mathrm{d}}{\mathrm{d} t} \int m^{2}\left(\left|\partial_{1} \partial_{t}^{2} u_{\mathrm{v}}\right|^{2}+\left|\operatorname{div}_{\mathrm{v}} \partial_{t}^{2} u_{\mathrm{v}}\right|^{2}\right) \mathrm{d} y-\lambda \int m m^{\prime} \partial_{t}^{2} u_{3} \operatorname{div} \partial_{t}^{3} u \mathrm{~d} y .
$$

Substituting the above two identities into (4.1) and using (1.5), we arrive at

$$
\frac{1}{2} \frac{\mathrm{d}}{\mathrm{d} t}\left(\left\|\sqrt{\bar{\rho}} \partial_{t}^{3} u\right\|_{0}^{2}-E\left(u_{t t}\right)\right)+\left\|\left(\sqrt{\mu_{1} J} \nabla_{\mathcal{A}} \partial_{t}^{3} u, \sqrt{\mu_{2} J} \operatorname{div}_{\mathcal{A}} \partial_{t}^{3} u\right)\right\|_{0}^{2}=\sum_{k=3}^{5} I_{k}^{H},
$$

where the terms on the right hand side can be bounded as follows, using (3.28), (3.21), (3.11) and (3.7).

$$
\begin{aligned}
I_{3}^{H} & \lesssim\left\|\partial_{t}^{3} \mathcal{N}\right\|_{0}\left\|\partial_{t}^{3} u\right\|_{0} \lesssim \sqrt{\mathcal{E}^{L}} \mathcal{D}^{H} \\
I_{4}^{H} & \lesssim\left\|N_{u}^{t, 3}\right\|_{0}\left\|\partial_{t}^{3} u\right\|_{0} \lesssim \sqrt{\mathcal{E}^{L}} \mathcal{D}^{H} \\
I_{5}^{H} & \lesssim\|J-1\|_{2}\left\|u_{t t}\right\|_{2}\left\|\partial_{t}^{3} u\right\|_{0} \lesssim \sqrt{\mathcal{E}^{L}} \mathcal{D}^{H} .
\end{aligned}
$$

Consequently, inserting the above three inequalities into (4.2) and using (3.69), we obtain the lemma.

Lemma 4.2. It holds that

$$
\begin{aligned}
& \frac{\mathrm{d}}{\mathrm{d} t}\left(\int \bar{\rho} J^{-1} \partial_{\mathrm{v}}^{j} \eta \cdot \partial_{\mathrm{v}}^{j} u \mathrm{~d} y+\frac{1}{2}\left\|\partial_{\mathrm{v}}^{j}\left(\sqrt{\mu_{1}} \nabla \eta, \sqrt{\mu_{2}} \operatorname{div} \eta\right)\right\|_{0}^{2}\right)+c\left\|\partial_{\mathrm{v}}^{j}\left(\partial_{1} \eta, \operatorname{div} \eta\right)\right\|_{0}^{2} \\
& \quad \lesssim\left\|\left(\partial_{1} \eta, \operatorname{div} \eta\right)\right\|_{j-1,0}^{2}+\left\|\partial_{\mathrm{v}}^{j} u\right\|_{0}^{2}+\left\|u_{t}\right\|_{j-1}^{2}+\sqrt{\mathcal{E}^{L}}\left(\|\eta\|_{7}^{2}+\mathcal{D}^{H}\right), \quad j=4, \ldots, 6 .
\end{aligned}
$$

Proof. We only prove the case $j=6$, while the rest cases can be shown in the same way. Following the process of deriving (3.40), we obtain

$$
\begin{aligned}
& \frac{\mathrm{d}}{\mathrm{d} t}\left(\int \bar{\rho} J^{-1} \partial_{\mathrm{v}}^{6} \eta \cdot \partial_{\mathrm{v}}^{6} u \mathrm{~d} y+\frac{1}{2}\left\|\partial_{\mathrm{v}}^{6}\left(\sqrt{\mu_{1}} \nabla \eta, \sqrt{\mu_{2}} \operatorname{div} \eta\right)\right\|_{0}^{2}\right)-E\left(\partial_{\mathrm{v}}^{6} \eta\right) \\
& \lesssim c\left(\left\|\partial_{\mathrm{v}}^{6} u\right\|_{0}^{2}+\left\|\partial_{\mathrm{v}}^{6}\left(\partial_{1} \eta, \operatorname{div} \eta\right)\right\|_{0}\left\|\left(\partial_{1} \eta, \operatorname{div} \eta\right)\right\|_{\underline{5}, 0}\right)+\sum_{k=1}^{4} J_{k}^{H},
\end{aligned}
$$

where

$$
\begin{aligned}
\sum_{k=1}^{4} J_{k}^{H}:= & \int \partial_{\mathrm{v}}^{6} \mathcal{N} \cdot \partial_{\mathrm{v}}^{6} \eta \mathrm{d} y+\int \partial_{\mathrm{v}}^{6} N_{u} \cdot \partial_{\mathrm{v}}^{6} \eta \mathrm{d} y \\
& -\sum_{k=0}^{5} \int\left(\partial_{\mathrm{v}}^{6}\left(\bar{\rho} J^{-1} u_{t}\right)-\bar{\rho} J^{-1} \partial_{\mathrm{v}}^{6} u_{t}\right) \cdot \partial_{\mathrm{v}}^{6} \eta \mathrm{d} y+\int \bar{\rho} J_{t}^{-1} \partial_{\mathrm{v}}^{6} \eta \cdot \partial_{\mathrm{v}}^{6} u \mathrm{~d} y,
\end{aligned}
$$


and $J_{1}^{H}, \cdots, J_{4}^{H}$ can be estimated as follows, using (3.33), (3.18), and (3.12), (3.11) and the smallness condition.

$$
\begin{aligned}
J_{1}^{H} & \lesssim\left\|\partial_{\mathrm{v}}^{5} \mathcal{N}\right\|_{0}\left\|\partial_{\mathrm{v}}^{6} \eta\right\|_{1} \lesssim \sqrt{\mathcal{E}^{L}}\|\eta\|_{7}^{2} \\
J_{2}^{H} & \lesssim\left\|\partial_{\mathrm{v}}^{5} N_{u}\right\|_{0}\left\|\partial_{\mathrm{v}}^{6} \eta\right\|_{1} \lesssim \sqrt{\mathcal{E}^{L}}\|(\eta, u)\|_{7}^{2} \\
J_{3}^{H} & \lesssim\left(\left\|J^{-1}-1\right\|_{6}+1\right)\left\|u_{t}\right\|_{5}\left\|\partial_{\mathrm{v}}^{6} \eta\right\|_{0} \lesssim\left\|u_{t}\right\|_{5}\left\|\partial_{\mathrm{v}}^{6} \eta\right\|_{0} \\
J_{4}^{H} & \lesssim\left\|J_{t}^{-1}\right\|_{2}\left\|\partial_{\mathrm{v}}^{6} u\right\|_{0}\left\|\partial_{\mathrm{v}}^{6} \eta\right\|_{0} \lesssim \sqrt{\mathcal{E}^{L}} \mathcal{D}^{H}
\end{aligned}
$$

Consequently, if we insert the above four inequalities into (4.3) and use (3.6), we obtain Lemma 4.2 .

Lemma 4.3. It holds that

$$
\begin{aligned}
& \frac{\mathrm{d}}{\mathrm{d} t}\left(\left\|\sqrt{\bar{\rho} J^{-1}} \partial_{\mathrm{v}}^{j} u\right\|_{0}^{2}-E\left(\partial_{\mathrm{v}}^{j} \eta\right)\right)+c\left\|\partial_{\mathrm{v}}^{j}(\nabla u, \operatorname{div} u)\right\|_{0}^{2} \\
& \quad \lesssim\left\|\left(\partial_{1} \eta, \operatorname{div} \eta\right)\right\|_{\underline{j-1,0}}^{2}+\left\|u_{t}\right\|_{j-1}^{2}+\sqrt{\mathcal{E}^{L}}\left(\|\eta\|_{7}^{2}+\mathcal{D}^{H}\right), \quad j=4, \ldots, 6 .
\end{aligned}
$$

Proof. We only prove the case $j=6$, since the rest cases are similar to deal with. Following the derivation of (3.49), one deduces that

$$
\frac{1}{2} \frac{\mathrm{d}}{\mathrm{d} t}\left(\left\|\sqrt{\bar{\rho} J^{-1}} \partial_{\mathrm{v}}^{6} u\right\|_{0}^{2}-E\left(\partial_{\mathrm{v}}^{6} \eta\right)\right)+\left\|\partial_{\mathrm{v}}^{6}\left(\sqrt{\mu_{1}} \nabla u, \sqrt{\mu_{2}} \operatorname{div} u\right)\right\|_{0}^{2} \leq c\left\|\left(\partial_{1} \eta, \operatorname{div} \eta\right)\right\|_{\underline{5}, 0}^{2}+\sum_{k=1}^{4} K_{k}^{H},
$$

where

$$
\begin{aligned}
\sum_{k=1}^{4} K_{k}^{H}:=\int & \partial_{\mathrm{v}}^{6} \mathcal{N} \cdot \partial_{\mathrm{v}}^{6} u \mathrm{~d} y+\int \partial_{\mathrm{v}}^{6} N_{u} \cdot \partial_{\mathrm{v}}^{6} u \mathrm{~d} y \\
& +\frac{1}{2} \int \bar{\rho} J_{t}^{-1}\left|\partial_{\mathrm{v}}^{6} u\right|^{2} \mathrm{~d} y-\sum_{k=0}^{5} \int \partial_{\mathrm{v}}^{6-k}\left(\bar{\rho} J^{-1}\right) \partial_{\mathrm{v}}^{k} u_{t} \cdot \partial_{\mathrm{v}}^{6} u \mathrm{~d} y
\end{aligned}
$$

On the other hand, similarly to (4.4)-(4.7), we have

$$
\sum_{k=1}^{4} K_{k}^{H} \lesssim\left\|u_{t}\right\|_{5}\left\|\partial_{\mathrm{v}}^{6} u\right\|_{0}+\sqrt{\mathcal{E}^{L}}\left(\|\eta\|_{7}^{2}+\mathcal{D}^{H}\right)
$$

Inserting the above estimate into (4.8), we can use (3.6) and Cauchy-Schwarz's inequality to obtain the desired estimate.

Lemma 4.4. It holds that

$$
\frac{\mathrm{d}}{\mathrm{d} t} \mathcal{H}_{5}(\eta)+\left\|\left(\partial_{1} \eta, \operatorname{div} \eta\right)\right\|_{6}^{2}+\|u\|_{7}^{2} \lesssim\left\|\left(\partial_{1} \eta, \operatorname{div} \eta, \nabla u\right)\right\|_{\underline{6}, 0}^{2}+\left\|u_{t}\right\|_{5}^{2}+\mathcal{E}^{L}\left(\|\eta\|_{7}^{2}+\mathcal{D}^{H}\right)
$$

where the energy functional $\mathcal{H}_{5}(\eta)$ satisfies $\left\|\partial_{1}^{2} \eta\right\|_{5}^{2}-h_{5}\left\|\partial_{1} \eta_{\mathrm{v}}\right\|_{6,0}^{2} \lesssim \mathcal{H}_{5}(\eta)$ and $h_{5}$ is a positive constant depending on $\Omega$ and the physical parameters in the perturbation equations.

Proof. The lemma easily follows from (3.33), (3.18) and (3.59). 
Lemma 4.5. We have

$$
\begin{aligned}
& \sum_{j=0}^{2}\left\|\partial_{t}^{j} u\right\|_{6-2 j}^{2} \lesssim\|\eta\|_{6}^{2}+\left\|\left(u, u_{t}, \partial_{t}^{3} u\right)\right\|_{0}^{2}+\mathcal{E}^{L} \mathcal{E}^{H}, \\
& \sum_{j=1}^{2}\left\|\partial_{t}^{j} u\right\|_{7-2 j}^{2} \lesssim\|u\|_{5}^{2}+\left\|\left(u_{t}, \partial_{t}^{3} u\right)\right\|_{1}^{2}+\mathcal{E}^{L} \mathcal{D}^{H} .
\end{aligned}
$$

Proof. (1) Taking $(i, j)=(4,0)$ in the Stokes estimate (3.61) and using (3.11), we find that

$$
\|u\|_{6}^{2} \lesssim\|\eta\|_{6}^{2}+\left\|u_{t}\right\|_{4}^{2}+S_{0,4}
$$

On the other hand, it follows from (3.32) and (3.18) that $S_{0,4}=\left\|\left(\mathcal{N}, N_{u}\right)\right\|_{4}^{2} \lesssim \mathcal{E}^{L} \mathcal{E}^{H}$. Therefore,

$$
\|u\|_{6}^{2} \lesssim\|\eta\|_{6}^{2}+\left\|u_{t}\right\|_{4}^{2}+\mathcal{E}^{L} \mathcal{E}^{H}
$$

If one utilizes (3.11), (2.22) 1 and the recursion formula (3.61) with $i=4$ for $j=1$ and 2 , one obtains

$$
\sum_{j=1}^{2}\left\|\partial_{t}^{j} u\right\|_{6-2 j}^{2} \lesssim \sum_{j=1}^{2}\left\|\partial_{t}^{j-1} u\right\|_{6-2 j}^{2}+\left\|\partial_{t}^{3} u\right\|_{0}^{2}+\sum_{j=1}^{2} S_{j, 4}
$$

On the other hand, from (3.34), (3.20), (3.19), (3.12) and (3.5) we get

$$
\sum_{j=1}^{2} S_{j, 4}=\sum_{j=1}^{2}\left(\left\|\partial_{t}^{j}\left(\mathcal{N}, N_{u}\right)\right\|_{4-2 j}^{2}+\left\|\bar{\rho} \sum_{k=0}^{j-1} \partial_{t}^{j-k} J^{-1} \partial_{t}^{k+1} u\right\|_{4-2 j}^{2}\right) \lesssim \mathcal{E}^{L} \mathcal{E}^{H}
$$

Thus,

$$
\sum_{j=1}^{2}\left\|\partial_{t}^{j} u\right\|_{6-2 j}^{2} \lesssim \sum_{j=1}^{2}\left\|\partial_{t}^{j-1} u\right\|_{6-2 j}^{2}+\left\|\partial_{t}^{3} u\right\|_{0}^{2}+\mathcal{E}^{L} \mathcal{E}^{H},
$$

which, together with (4.11), yields

$$
\sum_{j=0}^{2}\left\|\partial_{t}^{j} u\right\|_{6-2 j}^{2} \lesssim\|\eta\|_{6}^{2}+\sum_{j=1}^{2}\left\|\partial_{t}^{j-1} u\right\|_{6-2 j}^{2}+\left\|\partial_{t}^{3} u\right\|_{0}^{2}+\mathcal{E}^{L} \mathcal{E}^{H}
$$

Finally, employing the interpolation inequality, we get (4.9) from (4.13).

(2) We now turn to the proof of (4.10) for higher-order dissipation estimates. Making use of (3.11), (2.22) 1 and the recursion formula (3.61) with $i=5$ for $j=1$ to 2 , we obtain

$$
\sum_{j=1}^{2}\left\|\partial_{t}^{j} u\right\|_{7-2 j}^{2} \lesssim \sum_{j=1}^{2}\left\|\partial_{t}^{j-1} u\right\|_{7-2 j}^{2}+\left\|\partial_{t}^{3} u\right\|_{1}^{2}+\sum_{j=1}^{2} S_{j, 5}
$$

On the other hand, it follows from (3.35), (3.21), (3.12), (3.5) and the interpolation inequality that

$$
\begin{aligned}
\sum_{j=1}^{2} S_{j, 5} & =\sum_{j=1}^{2}\left(\left\|\partial_{t}^{j}\left(\mathcal{N}, N_{u}\right)\right\|_{5-2 j}^{2}+\left\|\bar{\rho} \sum_{k=0}^{j-1} \partial_{t}^{j-k} J^{-1} \partial_{t}^{k+1} u\right\|_{5-2 j}^{2}\right) \\
& \lesssim \mathcal{E}^{L} \mathcal{D}^{H}+\|u\|_{4}\left\|u_{t}\right\|_{2} \lesssim \mathcal{E}^{L} \mathcal{D}^{H} .
\end{aligned}
$$

Finally, exploiting the interpolation inequality again, we get (4.10) from (4.14) and (4.15). 
Now, we are ready to build the higher-order energy inequalities. In what follows the letters $c_{i}^{H}$, $i=1, \ldots, 8$, will denote generic constants which may depend on $\Omega$ and the physical parameters in the perturbation equations.

Proposition 4.1. Under the assumption (3.1), if $\delta$ is sufficiently small, then there is a norm $\tilde{\mathcal{E}}^{H}$, which is equivalent to $\mathcal{E}^{H}$, such that

$$
\frac{\mathrm{d}}{\mathrm{d} t} \tilde{\mathcal{E}}^{H}+\mathcal{D}^{H} \lesssim \sqrt{\mathcal{E}^{L}}\|\eta\|_{7}^{2}
$$

Proof. Similarly to (3.64), we employ Lemmas 4.24.3, (3.6) and the interpolation inequality to see that there is a constant $\kappa_{0,1}^{H} \geq 1$, such that

$$
\frac{\mathrm{d}}{\mathrm{d} t} \tilde{\mathcal{E}}_{1}^{H}+c_{1}^{H}\left\|\left(\partial_{1} \eta, \operatorname{div} \eta, \nabla u\right)\right\|_{\underline{6}, 0}^{2} \leq c_{2}^{H}\left(\left\|\left(\partial_{1} \eta, \operatorname{div} \eta\right)\right\|_{0}^{2}+\left\|u_{t}\right\|_{5}^{2}+\sqrt{\mathcal{E}^{L}}\left(\|\eta\|_{7}^{2}+\mathcal{D}^{H}\right)\right)
$$

for any $\kappa_{1}^{H} \geq \kappa_{0,1}^{H}$, where

$$
\begin{aligned}
\tilde{\mathcal{E}}_{1}^{H}:=\sum_{4 \leq \alpha_{1}+\alpha_{2} \leq 6}( & \left(\int \bar{\rho} J^{-1} \partial_{2}^{\alpha_{1}} \partial_{3}^{\alpha_{2}} \eta \cdot \partial_{2}^{\alpha_{1}} \partial_{3}^{\alpha_{2}} u \mathrm{~d} y-\kappa_{1}^{H} E\left(\partial_{2}^{\alpha_{1}} \partial_{3}^{\alpha_{2}} \eta\right)\right. \\
& \left.+\kappa_{1}^{H}\left\|\sqrt{\bar{\rho} J^{-1}} \partial_{2}^{\alpha_{1}} \partial_{3}^{\alpha_{2}} u\right\|_{0}+\frac{1}{2}\left\|\partial_{2}^{\alpha_{1}} \partial_{3}^{\alpha_{2}}\left(\sqrt{\mu_{1}} \nabla \eta, \sqrt{\mu_{2}} \operatorname{div} \eta\right)\right\|_{0}^{2}\right),
\end{aligned}
$$

and $\kappa_{0,1}^{H}$ depends on $\Omega$ and the physical parameters.

On the other hand, analogously to (3.65), we use (4.10) and the interpolation inequality to deduce from (4.16), Lemmas 4.4 and 4.1 that

$$
\begin{aligned}
& \frac{\mathrm{d}}{\mathrm{d} t}\left(\mathcal{H}_{5}(\eta)+c_{3}^{H} \tilde{\mathcal{E}}_{1}^{H}+c_{4}^{H}\left(\left\|\sqrt{\bar{\rho}} \partial_{t}^{3} u\right\|_{0}^{2}-E\left(u_{t t}\right)\right)\right)+c_{5}^{H}\left(\left\|\left(\partial_{1} \eta, \operatorname{div} \eta\right)\right\|_{6}^{2}+\|u\|_{7}^{2}+\left\|\partial_{t}^{3} u\right\|_{1}^{2}\right) \\
& \leq c_{6}^{H}\left(\left\|\left(\partial_{1} \eta, \operatorname{div} \eta\right)\right\|_{0}^{2}+\|u\|_{0}^{2}+\left\|u_{t}\right\|_{1}^{2}+\sqrt{\mathcal{E}^{L}}\left(\|\eta\|_{7}^{2}+\mathcal{D}^{H}\right)\right.
\end{aligned}
$$

which, together with Proposition 3.1 , implies that there is a constant $\kappa_{0,2}^{H} \geq 1$, such that

$$
\frac{\mathrm{d}}{\mathrm{d} t} \tilde{\mathcal{E}}_{2}^{H}+c_{7}^{H} \tilde{\mathcal{D}}^{H} \leq c_{6}^{H} \sqrt{\mathcal{E}^{L}}\left(\|\eta\|_{7}^{2}+\mathcal{D}^{H}\right) \quad \text { for any } \kappa_{2}^{H} \geq \kappa_{0,2}^{H},
$$

where

$$
\begin{gathered}
\tilde{\mathcal{E}}_{2}^{H}:=\mathcal{H}_{5}(\eta)+c_{3}^{H} \tilde{\mathcal{E}}_{1}^{H}+c_{4}^{H}\left(\left\|\sqrt{\bar{\rho}} \partial_{t}^{3} u\right\|_{0}^{2}-E\left(u_{t t}\right)\right)+\kappa_{2}^{H} \tilde{\mathcal{E}}^{L}, \\
\tilde{\mathcal{D}}^{H}:=\left\|\left(\partial_{1} \eta, \operatorname{div} \eta\right)\right\|_{6}^{2}+\|u\|_{7}^{2}+\left\|\left(u_{t}, \partial_{t}^{3} u\right)\right\|_{1}^{2} .
\end{gathered}
$$

Similarly to (3.67) and (3.68), we can utilize Lemma 4.5, (3.10) and the fact that

$$
\|\eta\|_{7} \text { is equivalent the norm }\|\eta\|_{7,0}+\left\|\partial_{1} \eta\right\|_{\underline{6}, 0}+\left\|\partial_{1}^{2} \eta\right\|_{5}
$$

to deduce that $\tilde{\mathcal{E}}_{2}^{H}$ is equivalent to $\mathcal{E}^{H}$ and $\mathcal{D}^{H} \leq c_{8}^{H} \tilde{\mathcal{D}}^{H}$ by choosing sufficiently large constants $\kappa_{1}^{H}$ and $\kappa_{2}^{H}$ which depend on $\Omega$ and the physical parameters. Consequently, one obtains the desired conclusion from (4.18) by defining $\tilde{\mathcal{E}}^{H}:=2 c_{8}^{H} \tilde{\mathcal{E}}_{2}^{H} / c_{7}^{H}$ and choosing $\delta \leq c_{7}^{H} / 2 c_{6}^{H} c_{8}^{H}$.

Finally, the following lemma will be needed in estimating the initial energy $\mathcal{E}^{H}(0)$ in the next section. 
Lemma 4.6. Under the assumption (3.1), if $\delta$ is sufficiently small, then $\mathcal{E}^{H} \lesssim \mathcal{E}$, where $\mathcal{E}:=$ $\|\eta\|_{7}^{2}+\|u\|_{6}^{2}$.

Proof. In view of (4.9), we have $\mathcal{E}^{H} \lesssim\|\eta\|_{7}^{2}+\left\|\left(u, u_{t}, \partial_{t}^{3} u\right)\right\|_{0}^{2}+\mathcal{E}^{L} \mathcal{E}^{H}$, which gives

$$
\mathcal{E}^{H} \lesssim\|\eta\|_{7}^{2}+\left\|\left(u, u_{t}, \partial_{t}^{3} u\right)\right\|_{0}^{2} \quad \text { for sufficiently small } \delta
$$

Next, we show that the $L^{2}$-norm of $u_{t}$ and $\partial_{t}^{3} u$ can be controlled by $\sqrt{\mathcal{E}}$.

Multiplying (3.25) $)_{2}$ with $j=2$ by $\partial_{t}^{3} u$ in $L^{2}$, we arrive at

$$
\begin{aligned}
\left\|\sqrt{\bar{\rho} J^{-1}} \partial_{t}^{3} u\right\|_{0}^{2}=\int & \left(\mu_{1} \Delta_{\mathcal{A}} u_{t t}+\mu_{2} \nabla_{\mathcal{A}} \operatorname{div}_{\mathcal{A}} u_{t t}+\nabla\left(P^{\prime}(\bar{\rho}) \operatorname{div}\left(\bar{\rho} u_{t}\right)\right)\right. \\
& \left.+g \operatorname{div}\left(\bar{\rho} u_{t}\right) e_{3}+\partial_{t}^{2} \mathcal{L}_{M}+\mathcal{N}_{t t}+N_{u}^{t, 2}\right) \cdot \partial_{t}^{3} u \mathrm{~d} y
\end{aligned}
$$

Employing (3.16), (3.10), (3.5) and (1.3), we see that

$$
\left\|\partial_{t}^{3} u\right\|_{0}^{2} \lesssim\left\|\left(u_{t}, u_{t t}\right)\right\|_{2}^{2}+\left\|\left(\mathcal{N}_{t t}, N_{u}^{t, 2}\right)\right\|_{0}^{2},
$$

which, together with (3.27) and (3.20), yields

$$
\left\|\partial_{t}^{3} u\right\|_{0}^{2} \lesssim\left\|u_{t}\right\|_{2}^{2}+\left\|u_{t t}\right\|_{2}^{2}+\mathcal{E}
$$

Here $\left\|u_{t t}\right\|_{2}$ can be bounded as follows, using (3.16) $,(3.8),(3.5)$ and $(3.25)_{2}$ with $j=1$.

$$
\begin{aligned}
\left\|u_{t t}\right\|_{2}^{2}= & \| \bar{\rho}^{-1} J\left(\mu_{1} \Delta_{\mathcal{A}} u_{t}+\mu_{2} \nabla_{\mathcal{A}} \operatorname{div}_{\mathcal{A}} u_{t}+\nabla\left(P^{\prime}(\bar{\rho}) \operatorname{div}(\bar{\rho} u)\right)\right. \\
& \left.+g \operatorname{div}(\bar{\rho} u) e_{3}+\partial_{t} \mathcal{L}_{M}+\mathcal{N}_{t}+N_{u}^{t, 1}\right) \|_{2}^{2} \\
\lesssim & \left\|u_{t}\right\|_{4}^{2}+\mathcal{E}+\left\|\mathcal{N}_{t}+N_{u}^{t, 1}\right\|_{2}^{2}
\end{aligned}
$$

which, together with (3.26) and (3.19), results in

$$
\left\|u_{t t}\right\|_{2}^{2} \lesssim\left\|u_{t}\right\|_{4}^{2}+\mathcal{E}
$$

Similarly, we can show that

$$
\left\|u_{t}\right\|_{4}^{2}=\left\|\bar{\rho}^{-1} J\left(\mu_{1} \Delta_{\mathcal{A}} u+\mu_{2} \nabla_{\mathcal{A}} \operatorname{div}_{\mathcal{A}} u+\nabla\left(P^{\prime}(\bar{\rho}) \operatorname{div}(\bar{\rho} \eta)\right)+g \operatorname{div}(\bar{\rho} \eta) e_{3}+\mathcal{L}_{M}+\mathcal{N}\right)\right\|_{4}^{2} \lesssim \mathcal{E}
$$

As a result, we infer from (4.20)- 4.22) that

$$
\left\|\partial_{t}^{3} u\right\|_{0}^{2} \lesssim \mathcal{E}
$$

Now, substituting (4.23) and (4.22) into (4.19), we obtain the lemma.

\section{Proof of Theorems 2.1 and 2.2}

This section is devoted to the proof of Theorems 2.1] and 2.2. Theorem 2.1 can be shown by establishing the stability estimate (2.24) and the local well-posedness of the transformed Parker problem. With Theorem 2.1 in hand, we can easily obtain Theorem 2.2 by transforming Lagrangian coordinates to Eulerian coordinates. 


\subsection{Stability estimate}

In this subsection we show the stability estimate (2.24) under the assumption

$$
\mathcal{G}_{3}(T):=\sup _{0 \leq \tau \leq T} \mathcal{E}^{H}(\tau)+\mathcal{G}_{2}(T) \leq \delta^{2}
$$

which is stronger than (3.1).

Proposition 4.1 implies

$$
\mathcal{G}_{1}(t) \lesssim \mathcal{E}^{H}(0)+\int_{0}^{t} \sqrt{\mathcal{E}^{L}(\tau)}\|\eta(\tau)\|_{7}^{2} \mathrm{~d} \tau
$$

Then we can use (5.1) to give

$$
\mathcal{G}_{1}(t) \lesssim \mathcal{E}^{H}(0)+\int_{0}^{t} \delta(1+\tau)^{-3 / 2}\|\eta(\tau)\|_{7}^{2} \mathrm{~d} \tau \lesssim \mathcal{E}^{H}(0)+\delta \mathcal{G}_{1}(t),
$$

which implies that

$$
\mathcal{G}_{1}(t) \lesssim \mathcal{E}^{H}(0)
$$

We now show the decay estimate of $\mathcal{G}_{2}(t)$. Note that $\mathcal{E}^{L}$ can be controlled by $\mathcal{D}^{L}$ except for $\|\eta\|_{4}$. To control $\|\eta\|_{4}$, we use the interpolation inequality (3.4) to get

$$
\|\eta\|_{4} \lesssim\|\eta\|_{3}^{\frac{3}{4}}\|\eta\|_{7}^{\frac{1}{4}}
$$

On the other hand, it follows from (5.2) and (3.67) that

$$
\mathcal{E}^{L}+\|\eta\|_{7}^{2} \lesssim \tilde{\mathcal{E}}^{L}+\|\eta\|_{7}^{2} \lesssim \mathcal{E}^{H}(0)
$$

whence,

$$
\tilde{\mathcal{E}}^{L} \lesssim \mathcal{E}^{L} \lesssim\left(\mathcal{D}^{L}\right)^{\frac{3}{4}}\left(\mathcal{E}^{L}+\|\eta\|_{7}^{2}\right)^{\frac{1}{4}} \lesssim\left(\mathcal{D}^{L}\right)^{\frac{3}{4}} \mathcal{E}^{H}(0)^{\frac{1}{4}}
$$

Inserting the above estimate into the lower-order energy inequality in Proposition 3.1 , we obtain

$$
\frac{\mathrm{d}}{\mathrm{d} t} \tilde{\mathcal{E}}^{L}+\frac{\left(\tilde{\mathcal{E}}^{L}\right)^{\frac{4}{3}}}{\mathcal{I}_{0}^{1 / 3}} \lesssim 0
$$

which implies

$$
\mathcal{E}^{L} \lesssim \tilde{\mathcal{E}}^{L} \lesssim \frac{\mathcal{I}_{0}}{\left(\left(\mathcal{I}_{0} / \mathcal{E}^{L}(0)\right)^{1 / 3}+t / 3\right)^{3}} \lesssim \frac{\mathcal{E}^{H}(0)}{1+t^{3}},
$$

where $\mathcal{I}_{0}:=c \mathcal{E}^{H}(0)$ for some positive constant $c$. Hence,

$$
\mathcal{G}_{2}(t) \lesssim \mathcal{E}^{H}(0)
$$

Now we add (5.2) to (15.3) to conclude $\mathcal{G}(t):=\mathcal{G}_{1}(t)+\mathcal{G}_{2}(t) \lesssim \mathcal{E}^{H}(0)$. On the other hand, thanks to Lemma 4.6, $\mathcal{E}^{H}(0) \lesssim\left\|\eta_{0}\right\|_{7}^{2}+\left\|u_{0}\right\|_{6}^{2}$. Therefore, $\mathcal{G}(t) \lesssim\left\|\eta_{0}\right\|_{7}^{2}+\left\|u_{0}\right\|_{6}^{2}$. Consequently, the stability estimate can be summarized as follows.

Proposition 5.1. Let $(\eta, u)$ be a solution of the transformed Parker problem (2.22), (2.23). Then there is a sufficiently small $\delta_{1}$, such that $(\eta, u)$ enjoys the following stability estimate:

$$
\mathcal{G}\left(T_{1}\right) \leq C_{1}\left(\left\|\eta_{0}\right\|_{7}^{2}+\left\|u_{0}\right\|_{6}^{2}\right)
$$

provided that $\sqrt{\mathcal{G}_{3}\left(T_{1}\right)} \leq \delta_{1}$ for some $T_{1}>0$, where $C_{1} \geq 1$ denotes a constant depending on $\Omega$ and the physical parameters in the perturbation equations. 


\subsection{Local well-posedness}

Now we introduce the local existence of a small classical solution to the transformed Parker problem.

Proposition 5.2. There exists a sufficiently small $\delta_{2}$, such that for any given initial data $\left(\eta_{0}, u_{0}\right) \in H^{7} \times H^{6}$ satisfying

$$
\sqrt{\left\|\eta_{0}\right\|_{7}^{2}+\left\|u_{0}\right\|_{6}^{2}} \leq \delta_{2}
$$

and compatibility conditions (i.e., $\left.\eta(\cdot, 0)\right|_{\partial \Omega}=0,\left.\partial_{t}^{j} u(\cdot, 0)\right|_{\partial \Omega}=0$ for $\left.j=0,1,2\right)$, there are a $T_{2}:=T_{2}\left(\delta_{2}\right)>0$, depending on $\delta_{2}, \Omega$ and the known physical parameters, and a unique classical solution $(\eta, u) \in C^{0}\left(\left[0, T_{2}\right], H^{7} \times H^{6}\right)$ to the transformed Parker problem (2.22), (2.23). Moreover, $\partial_{t}^{i} u \in C^{0}\left(\left[0, T_{2}\right], H^{6-2 i}\right)$ for $1 \leq i \leq 3, \mathcal{E}^{H}(0) \lesssim\left\|\eta_{0}\right\|_{7}^{2}+\left\|u_{0}\right\|_{6}^{2}$, and

$$
\sup _{0 \leq \tau \leq T_{2}} \mathcal{E}^{H}(\tau)+\int_{0}^{T_{2}} \mathcal{D}^{H}(\tau) \mathrm{d} \tau<\infty,
$$

and $\mathcal{G}(t)$ is a continuous function on $\left[0, T_{2}\right]$.

Remark 5.1. In view of the definition of $\mathcal{G}(t)$, we have

$$
\mathcal{G}(0) \leq 2 \mathcal{E}^{H}(0) \leq C_{2}\left(\left\|\eta_{0}\right\|_{7}^{2}+\left\|u_{0}\right\|_{6}^{2}\right)
$$

where $C_{2} \geq 1$ denotes a constant depending on $\Omega$ and the physical parameters.

Proof. The transformed Parker problem is very similar to the surface wave problem (1.4) in [19]. Moreover, our problem indeed is simpler than the the surface wave problem due to the non-slip boundary condition $\left.u\right|_{\partial \Omega}=0$. Using a standard iteration method as in [19], we can easily prove Proposition 5.2, and hence we omit the proof here. In addition, the continuity, such as $(\eta, u) \in C^{0}\left(\left[0, T_{2}\right], H^{7} \times H^{6}\right), \mathcal{G}(t)$ and so on, can be verified by using the regularity of $(\eta, u)$, the transformed perturbation equations and a standard regularized method.

\subsection{Proof of Theorem 2.1}

Now we are in a position to show Theorem 2.1. Let the initial data $\left(\eta_{0}, u_{0}\right) \in H^{7} \times H^{6}$ satisfy the assumptions in Theorem 2.1, where $\delta$ in Theorem 2.1 further satisfies

$$
\delta \leq \frac{\min \left\{\delta_{1}, \delta_{2}\right\}}{2 C_{1} C_{2}}
$$

and $\delta_{1}, \delta_{2}$ are the same as in Propositions 5.1 and 5.2 respectively. Then, by Proposition 5.2 , there exists a solution $(\eta, u)$, defined on $\Omega \times\left(0, T^{\text {max }}\right)$, to the transformed Parker problem where $T^{\max }$ denotes the maximal existence time, such that $\mathcal{G}(t)$ is continuous for any $t \in\left[0, T^{\max }\right)$. Obviously, $T^{\max } \geq T_{2}\left(\delta_{2}\right)$. We denote

$$
T^{*}=\sup \left\{t \in\left(0, T^{\max }\right) \mid \sqrt{\mathcal{G}(t)} \leq \min \left\{\delta_{1}, \delta_{2}\right\}\right\}
$$

then $0<T^{*} \leq T^{\max }$ by (5.5) and the continuity of $\mathcal{G}(t)$. Next we show $T^{*}=\infty$ by contradiction.

Assume $T^{*}<\infty$. By virtue of Proposition [5.2, $T^{\max }>T^{*}$. Then one has

$$
\mathcal{G}\left(T^{*}\right)=\min \left\{\delta_{1}, \delta_{2}\right\}
$$


by the continuity of $\mathcal{G}(t)$ on $\left(0, T^{\text {max }}\right)$. On the other hand, noting that $\sqrt{\mathcal{G}_{3}\left(T^{*}\right)} \leq \sqrt{\mathcal{G}\left(T^{*}\right)} \leq \delta_{1}$, we see that $(\eta, u)$ satisfies (5.4) with $T^{*}$ in place of $T_{1}$. Thus, by the conditions $\sqrt{\left\|\eta_{0}\right\|_{7}^{2}+\left\|u_{0}\right\|_{6}^{2}} \leq$ $\delta$ and (․․6), we further obtain a more precise estimate

$$
\sqrt{\mathcal{G}\left(T^{*}\right)} \leq \min \left\{\delta_{1}, \delta_{2}\right\} / 2
$$

which is a contradiction. Hence $T^{*}=\infty$, and we obtain a global solution $(\eta, u)$ to the transformed Parker problem, which satisfies (2.24). Finally, the uniqueness of the global solution $(\eta, u)$ can be easily verified by the standard energy method. This completes the proof of Theorem 2.1.

\subsection{Proof of Theorem 2.2}

To show Theorem [2.2, let $\left(\varrho_{0}, v_{0}, N_{0}\right)$ satisfy the assumptions of Theorem 2.2. Then, for sufficiently small $\delta<0$, we can use the standard iteration method as in [29, Proposition 3.1] to see that there are a $T>0$ and a unique classical solution $(\varrho, v, N) \in C^{0}\left([0, T], H^{6}\right)$ of the original Parker problem. Moreover, $\partial_{t}^{i} v \in C^{0}\left([0, T], H^{6-2 i}\right)$ for $1 \leq i \leq 3$, and

$$
\sup _{0 \leq t \leq T}\left(\|(\varrho, N)\|_{6}^{2}+\sum_{k=0}^{3}\left\|\partial_{t}^{k} v(t)\right\|_{6-2 k}^{2}\right)+\int_{0}^{T} \sum_{k=0}^{3}\left\|\partial_{t}^{k} v(t)\right\|_{7-2 k}^{2} \mathrm{~d} t<\infty .
$$

Let $u_{0}(y)=v_{0}\left(\zeta_{0}(y)\right)$, where $\zeta_{0}$ is given by Theorem 2.2. Noting that $\left\|u_{0}\right\|_{6}^{2} \lesssim\left\|v_{0}\right\|_{6}^{2}$ by the first two initial conditions in Theorem 2.2 for sufficiently small $\delta$, one sees that $\eta_{0}:=\zeta_{0}-y$ and $u_{0}$ satisfy the first two conditions in Theorem 2.1, and $\left.u_{0}\right|_{\partial \Omega}=0$ by the the first two conditions in Theorem 2.2. On the other hand, employing the last two conditions in Theorem 2.2, we can moreover verify that $\left(\eta_{0}, u_{0}\right)$ satisfies the third condition in Theorem 2.1 by following the derivation of $(2.22)_{2}$ from $(1.11)_{2}$. Hence, $\left(\eta_{0}, u_{0}\right)$ satisfies the three conditions in Theorem 2.1 for sufficiently small $\delta$.

Now one can use the initial data $\left(\eta_{0}, u_{0}\right)$ and Theorem 2.1 to construct a classical unique solution $(\tilde{\eta}, \tilde{u}) \in C\left(\mathbb{R}_{0}^{+}, H^{7} \times H^{6}\right)$ to the transformed Parker problem, which satisfies the stability estimate (2.24) as $(\eta, u)$. Thus, we can choose a sufficiently small $\delta$, so that there exists a continuously differential invertible function $\tilde{\zeta}^{-1}$ of $\tilde{\zeta}:=y+\tilde{\eta}$. Let

$$
\tilde{\sigma}:=\left.\left(\bar{\rho}(\operatorname{det} \nabla \tilde{\zeta})^{-1}\right)\right|_{y=\tilde{\zeta}^{-1}}-\bar{\rho}\left(x_{3}\right), \quad \tilde{v}:=\tilde{u}\left(\tilde{\zeta}^{-1}, t\right), \quad \tilde{N}:=\left.\left(m(\operatorname{det} \nabla \tilde{\zeta})^{-1} \partial_{1} \tilde{\zeta}\right)\right|_{y=\tilde{\zeta}^{-1}}-m e_{3}
$$

In view of the relations

$$
\begin{aligned}
& 1 \lesssim \operatorname{det} \nabla \tilde{\zeta} \lesssim 1 \\
& \partial_{t}\left(\left.f(y, t)\right|_{y=\tilde{\zeta}^{-1}}\right)=\left.\partial_{t} f(y, t)\right|_{y=\tilde{\zeta}^{-1}}+\left.\partial_{i} f(y, t)\right|_{y=\tilde{\zeta}^{-1}} \partial_{t} \tilde{\zeta}_{i}^{-1} \\
& \partial_{t} \tilde{\zeta}^{-1}=-\left.(\nabla \tilde{\zeta})^{-1}\right|_{y=\tilde{\zeta}^{-1}} \tilde{v} \text { and } \nabla \tilde{\zeta}^{-1}=\left.(\nabla \tilde{\zeta})^{-1}\right|_{y=\tilde{\zeta}^{-1}}
\end{aligned}
$$

we see that $(\tilde{\sigma}, \tilde{v}, \tilde{N})$ is a classical solution to the original Parker problem. Moreover, by a standard uniqueness proof, we have $(\tilde{\sigma}, \tilde{v}, \tilde{N})=(\varrho, v, N)$, and therefore, one obtains the existence of a unique global solution in Theorem 2.2 . Finally, recalling that $(\tilde{\eta}, \tilde{u})$ satisfies the stability estimate (2.24) as $(\eta, u)$, we make use the relations (5.8)-(5.11) to deduce the stability estimate (2.30). This completes the proof of Theorem 2.2 . 


\section{Proof of Theorem 2.3}

In this section we prove Theorem 2.3 . To begin with, we introduce the instability of the linearized Parker problem (1.12)-(1.14) and the local well-posedness of strong solutions to the original Parker problem (1.11)-(1.13).

Proposition 6.1. Under the assumptions of Theorem 2.3, the equilibrium state $(\bar{\rho}, 0, \bar{M})$ of the linearized Parker problem (1.12)-(1.14) is unstable, that is, there is an unstable solution in the form

$$
(\varrho, v, N):=e^{\Lambda t}\left(-\operatorname{div}(\bar{\rho} \tilde{u}) / \Lambda, \tilde{u},\left(m \partial_{1} \tilde{u}-\tilde{u}_{3} \bar{M}^{\prime}-\bar{M} \operatorname{div} \tilde{u}\right) / \Lambda\right)
$$

of (1.12) -(1.14), where $\tilde{u} \in H^{4} \cap \mathcal{A}$ solves the boundary value problem:

$$
\left\{\begin{array}{c}
\Lambda^{2} \bar{\rho} \tilde{u}=g \bar{\rho}^{\prime} \tilde{u}_{3} e_{3}+\nabla\left(P^{\prime}(\bar{\rho}) \operatorname{div}(\bar{\rho} \tilde{u})\right)+g \bar{\rho} \operatorname{div} \tilde{u} e_{3}+\lambda m\left(m \partial_{1}^{2} \tilde{u}-\bar{M} \operatorname{div} \partial_{1} \tilde{u}\right) \\
\quad+\nabla\left(\lambda m\left(m \partial_{2} \tilde{u}_{2}+m \partial_{3} \tilde{u}_{3}+m^{\prime} \tilde{u}_{3}\right)\right)+\Lambda \mu_{1} \Delta \tilde{u}+\Lambda \mu_{2} \nabla \operatorname{div} \tilde{u}, \\
\left.\tilde{u}\right|_{\partial \Omega}=0
\end{array}\right.
$$

with $\Lambda>0$ being a constant satisfying

$$
\Lambda^{2}=\sup _{w \in \mathcal{A}} \mathcal{E}_{\mathrm{c}}(w, \Lambda)=\mathcal{E}_{\mathrm{c}}(\tilde{u}, \Lambda)
$$

where

$$
\mathcal{A}:=\left\{w \in H_{0}^{1} \mid \int \bar{\rho} w^{2} \mathrm{~d} x=1\right\}
$$

and

$$
\mathcal{E}_{\mathrm{c}}(w, \Lambda):=E(w)-\Lambda \int\left(\mu_{1}|\nabla w|^{2}+\mu_{2}|\operatorname{div} w|^{2}\right) \mathrm{d} x
$$

Moreover,

$$
\tilde{u}_{3} \neq 0
$$

Proof. Proposition 6.1 has been proved in [27, Theorem 2.1], where the considered domain is a bounded $C^{2}$-domain and $g$ is a positive constant. Next, we denote the the bounded $C^{2}$-domain in [27, Theorem 2.1] by $\Omega^{\prime}$, and show how to modify the proof in [27] to establish Proposition 6.1 in a strip domain $\Omega$ here.

(1) Since $\Omega^{\prime}$ is a $C^{2}$-bounded domain, $H^{1}\left(\Omega^{\prime}\right)$ is compactly embedded in $L^{2}\left(\Omega^{\prime}\right)$. In the derivation of the first conclusion in [27, Proposition 2.1], Jiang et.al. used this fact to derive that

$$
\sup _{w \in \mathcal{A}} \mathcal{E}_{\mathrm{c}}(w, s) \leq \mathcal{E}_{\mathrm{c}}(\tilde{w}, s) \text { for the bounded domain } \Omega^{\prime}
$$

and

$$
\int_{\Omega^{\prime}} \bar{\rho} \tilde{w}^{2} \mathrm{~d} x=1
$$

where $\tilde{w}$ is the limit of a subsequence of a maximizing sequence $\left\{w_{n}\right\}_{n=1}^{\infty}$ of $\sup _{w \in \mathcal{A}} \mathcal{E}_{\mathrm{c}}(w, s)$. More precisely, $\left\{w_{n}\right\}_{n=1}^{\infty} \ni w_{n_{m}} \rightarrow \tilde{w}$ weakly in $H_{0}^{1}\left(\Omega^{\prime}\right)$ and

$$
w_{n_{m}} \rightarrow \tilde{w} \text { strongly in } L^{2}\left(\Omega^{\prime}\right) \text { as } n_{m} \rightarrow \infty \text {. }
$$

Since $g \in C_{0}^{6}(\mathbb{R})$ and (6.6) holds for a bounded subdomain of an unbounded domain, (6.4) still holds for a strip domain $\Omega$. However, by the lower semi-continuity of weak convergence, we only have $\int_{\Omega} \bar{\rho} \tilde{w}^{2} \mathrm{~d} x \leq 1$. 
To show (6.5) with $\Omega$ in place of $\Omega^{\prime}$, we argue by contradiction. From the instability condition $\Xi>0$ we get for sufficiently small $s$ that $\sup _{w \in \mathcal{A}} \mathcal{E}_{\mathrm{c}}(w, s)>0$, which implies $\int_{\Omega} \bar{\rho} \tilde{w}^{2} \mathrm{~d} x \neq 0$. Now we assume that $\int_{\Omega} \bar{\rho} \tilde{w}^{2} \mathrm{~d} x<1$, then

$$
\mathcal{E}_{\mathrm{c}}(\tilde{w}, s)<\frac{\mathcal{E}_{\mathrm{c}}(\tilde{w}, s)}{\int_{\Omega} \bar{\rho} \tilde{w}^{2} \mathrm{~d} x} \leq \sup _{w \in \mathcal{A}} \mathcal{E}_{\mathrm{c}}(w, s) \leq \mathcal{E}_{\mathrm{c}}(\tilde{w}, s),
$$

which is a contradiction. Hence, $\int_{\Omega} \bar{\rho} \tilde{w}^{2} \mathrm{~d} x=1$.

(2) In view of the proof of Proposition 4.2, we observe that $u \in H^{4}\left(\Omega^{\prime}\right)$ if $u \in H_{0}^{1}$ is a weak solution of the boundary value problem (6.1) with a bounded $C^{4}$-domain $\Omega^{\prime}$ in place of $\Omega$. This conclusion also holds for the strip domain $\Omega$ by employing the same domain expansion technique as in the derivation of (3.7) in [31].

(3) Jiang et.al. in [27] used Poincaré's inequality

$$
\|w\|_{0} \lesssim\|\nabla w\|_{0} \quad \text { for any } w \in H_{0}^{1}\left(\Omega^{\prime}\right)
$$

to derive (3.7). Obviously, this inequality still holds for the strip domain $\Omega$, see (3.6).

With the above facts, we easily establish Proposition 6.1 by following the proof of [27, Theorem $2.1]$.

Proposition 6.2. Under the assumptions of Theorem 2.3, for any given initial data $\left(\varrho_{0}, v_{0}, N_{0}\right) \in$ $H^{3}$ satisfying $\inf _{x \in \Omega}\left\{\left(\varrho_{0}+\bar{\rho}\right)(x)\right\}>0$, the compatibility conditions $\left.\partial_{t}^{i} v(\cdot, 0)\right|_{\partial \Omega}=0$ for $i=0,1$, and $\operatorname{div} N_{0}=0$, there exist $a T^{\max }>0$ and a unique classical solution $(\varrho, v, N) \in C^{0}\left(\left[0, T^{\max }\right), H^{3}\right)$ to the original Parker problem, where $(\varrho, v, N)$ enjoys the following properties:

$$
v_{t} \in C^{0}\left(\left[0, T^{\max }\right), H^{1}\right) \cap L^{2}\left(\left(0, T^{\max }\right), H^{2}\right) \quad \text { and } \quad 0<\inf _{(x, t) \in \Omega \times\left(0, T^{\max }\right)}\{\varrho(x, t)+\bar{\rho}\},
$$

where $T^{\max }$ denotes the maximal time of existence of the solution $(\varrho, v, N)$. In addition, if

$$
\inf _{x \in \Omega}\{\bar{\rho}(x)\} / 2 \leq(\varrho+\bar{\rho})(x, t) \leq 2 \sup _{x \in \Omega}\{\bar{\rho}(x)\} \quad \text { on } \Omega \times\left(0, T^{\max }\right),
$$

then

$$
\begin{aligned}
& \mathcal{S}(\varrho(t), v(t), N(t)):=\sqrt{\|v(t)\|_{1}^{2}+\left\|\left(\varrho(t), N(t), \varrho_{t}, v_{t}, N_{t}\right)\right\|_{0}^{2}} \\
& \leq C_{3} \sqrt{\|(\varrho, N)(t)\|_{1}^{2}+\|v(t)\|_{2}^{2}+\|(\varrho, v, N)(t)\|_{1}^{2}\|(\varrho, v, N)(t)\|_{2}^{2}}
\end{aligned}
$$

for any $t \in\left(0, T^{\max }\right)$, where the constant $C_{3} \geq 1$ only depends on $\Omega$ and the known physical parameters in the perturbation equations.

Proof. Proposition 6.2 can be established by a standard iteration scheme as in [29, Proposition 3.1], and hence, we omit its proof here which involves only tedious calculations. Here we only show (6.8).

Recalling (1.6) and the relation

$$
P(\varrho+\bar{\rho})=\bar{P}+P^{\prime}(\bar{\rho}) \varrho+\int_{0}^{\varrho}(\varrho-z) P^{\prime \prime}(z+\bar{\rho}) \mathrm{d} z,
$$

we can write $(1.11)_{2}$ as follows

$$
\begin{aligned}
& (\varrho+\bar{\rho}) v_{t}+(\varrho+\bar{\rho}) v \cdot \nabla v+\nabla\left(P^{\prime}(\bar{\rho}) \varrho+\int_{0}^{\varrho}(\varrho-z) P^{\prime \prime}(z+\bar{\rho}) \mathrm{d} z+\lambda\left(2 m N_{1}+|N|^{2}\right) / 2\right) \\
& =\mu_{1} \Delta v+\mu_{2} \nabla \operatorname{div} v+\lambda\left(\bar{M}^{\prime} N_{3}+m \partial_{1} N+N \cdot \nabla N\right)-\varrho g e_{3} .
\end{aligned}
$$


Multiplying the above identity by $v_{t}$ in $L^{2}$ and using (6.7), we deduce that, for any $\epsilon>0$,

$$
\begin{aligned}
\int(\varrho+\bar{\rho})\left|v_{t}\right|^{2} \mathrm{~d} x \leq & C_{\epsilon}\left(\|(\varrho, N)\|_{1}^{2}+\|v\|_{2}^{2}\right. \\
& \left.+\|(\varrho, v, N)\|_{1}^{2}\|(\varrho, v, N)\|_{2}^{2}\right)+\epsilon\left\|v_{t}\right\|_{0}^{2}
\end{aligned}
$$

where the constant $C_{\epsilon}$ depends on $\epsilon$. So, one can use (6.7) again to get

$$
\left\|v_{t}\right\|_{0}^{2} \leq\|v\|_{2}^{2}+\|(\varrho, N)\|_{1}^{2}+\|(\varrho, v, N)\|_{1}^{2}\|(\varrho, v, N)\|_{2}^{2} .
$$

Using $(1.11)_{1}$ and $(1.11)_{3}$, we have

$$
\left\|\varrho_{t}\right\|_{0} \lesssim\left(1+\|\varrho\|_{2}\right)\|v\|_{1}, \quad\left\|N_{t}\right\|_{0} \lesssim\left(1+\|N\|_{2}\right)\|v\|_{1}
$$

From the above three estimates we get (6.8) immediately.

We next use Propositions 6.1 6.2 to construct unstable solutions to the original Parker problem. To this end, we first use Proposition 6.1 to give a solution of the linearized perturbation equations (1.14) in the form:

$$
\left(\varrho^{1}, v^{1}, N^{1}\right)=e^{\Lambda t}\left(\tilde{\varrho}_{0}, \tilde{v}_{0}, \tilde{N}_{0}\right) \in H^{3}
$$

where

$$
\left(\tilde{\varrho}_{0}, \tilde{v}_{0}, \tilde{N}_{0}\right):=\left(-\operatorname{div}(\bar{\rho} \tilde{u}) / \Lambda, \tilde{u},\left(m \partial_{1} \tilde{u}-\tilde{u}_{3} \bar{M}^{\prime}-\bar{M} \operatorname{div} \tilde{u}\right) / \Lambda\right)
$$

Now, denote

$$
\left(\varrho^{\mathrm{a}}, v^{\mathrm{a}}, N^{\mathrm{a}}\right):=\delta\left(\varrho^{\mathrm{l}}, v^{\mathrm{l}}, N^{\mathrm{l}}\right), \quad \delta>0, \text { a constant. }
$$

Then, $\left(\varrho^{\mathrm{a}}, v^{\mathrm{a}}, N^{\mathrm{a}}\right)$ solves the linearized perturbation equations (1.14) with initial data $\delta\left(\tilde{\varrho}_{0}, \tilde{v}_{0}, \tilde{N}_{0}\right)$, and satisfies

$$
\left\|\left(\varrho^{\mathrm{a}}, v^{\mathrm{a}}, N^{\mathrm{a}}\right)\right\|_{3} \lesssim \delta e^{\Lambda t} .
$$

In the rest of this paper, we call (6.10) an approximate solution of the original Parker problem for fixed $\delta$.

Secondly, we modify the initial data $\delta\left(\tilde{\varrho}_{0}, \tilde{v}_{0}, \tilde{N}_{0}\right)$ to construct a solution of the original Parker problem for sufficiently small $\delta>0$.

Lemma 6.1. Let $\left(\tilde{\varrho}_{0}, \tilde{v}_{0}, \tilde{N}_{0}\right)$ be the same as in (6.9). Then there are an error function $v_{\mathrm{r}}$ and a constant $\delta_{1} \in(0,1)$ depending on $\left(\tilde{\varrho}_{0}, \tilde{v}_{0}, \tilde{N}_{0}\right)$, such that for any $\delta \in\left(0, \delta_{1}\right)$,

(1) The modified initial data

$$
\left(\varrho_{0}^{\delta}, v_{0}^{\delta}, N_{0}^{\delta}\right):=\delta\left(\tilde{\varrho}_{0}, \tilde{v}_{0}, \tilde{N}_{0}\right)+\left(0, \delta^{2} v_{\mathrm{r}}, 0\right)
$$

satisfies the compatibility conditions on boundary of the Parker problem (1.11)-(1.13).

(2) $v_{\mathrm{r}}$ satisfies the following estimate:

$$
\left\|v_{\mathrm{r}}\right\|_{3} \leq C_{4}
$$

where the constant $C_{4} \geq 1$ depends on $\left\|\left(\tilde{\varrho}_{0}, \tilde{v}_{0}, \tilde{N}_{0}\right)\right\|_{3}$ and other physical parameters, but is independent of $\delta$. 
Proof. Note that $\left(\tilde{\varrho}_{0}, \tilde{v}_{0}, \tilde{N}_{0}\right)$ satisfies

$$
\left.\tilde{v}_{0}\right|_{\partial \Omega}=0,\left.\quad\left(\mu_{1} \Delta \tilde{v}_{0}+\mu_{2} \nabla \operatorname{div} \tilde{v}_{0}+\lambda \bar{M}^{\prime} \tilde{N}_{3}^{0}+\lambda m \partial_{1} \tilde{N}_{0}-\tilde{\varrho}_{0} g e_{3}-\nabla\left(P^{\prime}(\bar{\rho}) \tilde{\varrho}_{0}+\lambda m \tilde{N}_{1}^{0}\right)\right)\right|_{\partial \Omega}=0 .
$$

Hence, if the modified initial data satisfy (6.12), then we expect $v_{\mathrm{r}}$ to satisfy the following problem:

$$
\left\{\begin{array}{l}
\mu_{1} \Delta v_{\mathrm{r}}+\mu_{2} \nabla \operatorname{div} v_{\mathrm{r}}-\delta^{2}\left(\delta \tilde{\varrho}_{0}+\bar{\rho}\right) v_{\mathrm{r}} \cdot \nabla v_{\mathrm{r}}-\delta\left(\delta \tilde{\varrho}_{0}+\bar{\rho}\right) v_{\mathrm{r}} \cdot \nabla \tilde{v}_{0} \\
\quad=\nabla\left(\int_{0}^{\tilde{\varrho}_{0}}\left(\tilde{\varrho}_{0}-z\right) P^{\prime \prime}(\delta z+\bar{\rho}) \mathrm{d} z+\frac{\lambda\left|\tilde{N}_{0}\right|^{2}}{2}\right)-\lambda \tilde{N}_{0} \cdot \nabla \tilde{N}_{0}=: F\left(\tilde{\varrho}_{0}, \tilde{v}_{0}, \tilde{N}_{0}\right), \\
\left.v_{\mathrm{r}}\right|_{\partial \Omega}=0 .
\end{array}\right.
$$

Thus the modified initial data naturally satisfy the compatibility conditions on boundary.

Next we shall look for a solution $v_{\mathrm{r}}$ to the boundary problem (6.13) when $\delta$ is sufficiently small. We begin with the linearization of (6.13) which reads as

$$
\mu_{1} \Delta v_{\mathrm{r}}+\mu_{2} \nabla \operatorname{div} v_{\mathrm{r}}=F\left(\tilde{\varrho}_{0}, \tilde{v}_{0}, \tilde{N}_{0}\right)+\delta^{2}\left(\delta \varrho_{0}+\bar{\rho}\right) w \cdot \nabla w+\delta\left(\delta \tilde{\varrho}_{0}+\bar{\rho}\right) w \cdot \nabla \tilde{v}_{0}
$$

with boundary condition

$$
\left.v_{\mathrm{r}}\right|_{\partial \Omega}=0 .
$$

Let $w \in H^{3}$, then it follows from the elliptic theory that there is a solution $v_{\mathrm{r}}$ of $(6.14)-(6.15)$ satisfying

$$
\left\|v_{\mathrm{r}}\right\|_{3} \leq\left\|F\left(\tilde{\varrho}_{0}, \tilde{v}_{0}, \tilde{N}_{0}\right)+\delta^{2}\left(\delta \tilde{\varrho}_{0}+\bar{\rho}\right) w \cdot \nabla w+\delta\left(\delta \tilde{\varrho}_{0}+\bar{\rho}\right) w \cdot \nabla \tilde{v}_{0}\right\|_{1} \leq c_{1}\left(1+\delta^{2}\|w\|_{2}^{2}\right), \quad \delta \in(0,1),
$$

where $c_{1}$ depends on $\left\|\left(\varrho_{0}, \tilde{v}_{0}, \tilde{N}_{0}\right)\right\|_{3}$ and the physical parameters. Now, taking $\delta \leq \min \left\{\left(2 c_{1}\right)^{-1}, 1\right\}$, we have for any $\|w\|_{3} \leq 2 c_{1}$ that $\left\|v_{\mathrm{r}}\right\|_{3} \leq 2 c_{1}$. Therefore, one can construct an approximate function sequence $v_{\mathrm{r}}^{n}$, such that

$$
\mu_{1} \Delta v_{\mathrm{r}}^{n+1}+\mu_{2} \nabla \operatorname{div} v_{\mathrm{r}}^{n+1}-\delta^{2}\left(\delta \tilde{\varrho}_{0}+\bar{\rho}\right) v_{\mathrm{r}}^{n} \cdot \nabla v_{\mathrm{r}}^{n}-\delta\left(\delta \tilde{\varrho}_{0}+\bar{\rho}\right) v_{\mathrm{r}}^{n} \cdot \nabla \tilde{v}_{0}=F\left(\tilde{\varrho}_{0}, \tilde{v}_{0}, \tilde{N}_{0}\right),
$$

and for any $n,\left\|v_{\mathrm{r}}^{n}\right\|_{3} \leq 2 c_{1}$ and $\left\|v_{\mathrm{r}}^{n+1}-v_{\mathrm{r}}^{n}\right\|_{3} \leq c_{2} \delta\left\|v_{\mathrm{r}}^{n}-v_{\mathrm{r}}^{n-1}\right\|_{3}$ for some constant $c_{2}$ independent of $\delta$ and $n$.

Finally, we choose a sufficiently small $\delta$ so that $c_{2} \delta<1$, and use then a compactness argument to get a limit function which solves the nonlinear boundary problem (6.13). Moreover $\left\|v_{\mathrm{r}}\right\|_{3} \leq 2 c_{1}$. Thus we have proved Lemma 6.1.

Now, in view of the condition $\inf _{x \in \Omega}\{\bar{\rho}(x)\}>0$ and the embedding inequality (3.3), we can choose a sufficiently small $\tau \in\left(0, \delta_{1}\right)$, such that

$$
\frac{\inf _{x \in \Omega}\{\bar{\rho}(x)\}}{2} \leq \inf _{x \in \Omega}\left\{\varrho_{0}^{\delta}(x)+\bar{\rho}(x)\right\} \leq 2 \sup _{x \in \Omega}\{\bar{\rho}(x)\} \quad \text { for any } \delta \in(0, \tau) .
$$

Thus, by virtue of Proposition 6.2, for any given $\delta<\tau$, there exists a unique local-in-time classical solution $\left(\varrho^{\delta}, v^{\delta}, N^{\delta}\right) \in C^{0}\left(\left[0, T^{\max }\right), H^{3}\right)$ to the original Parker problem, emanating from the initial data $\left(\varrho_{0}^{\delta}, v_{0}^{\delta}, N_{0}^{\delta}\right)$.

Thirdly, we estimate the error between the approximate solution $\left(\varrho^{\mathrm{a}}, v^{\mathrm{a}}, N^{\mathrm{a}}\right)$ and $\left(\varrho^{\delta}, v^{\delta}, N^{\delta}\right)$. Denote $\left(\varrho^{\mathrm{d}}, v^{\mathrm{d}}, N^{\mathrm{d}}\right)=\left(\varrho^{\delta}, v^{\delta}, N^{\delta}\right)-\left(\varrho^{\mathrm{a}}, v^{\mathrm{a}}, N^{\mathrm{a}}\right)$. Then $\left(\varrho^{\mathrm{d}}, v^{\mathrm{d}}, N^{\mathrm{d}}\right)$ satisfies the following error equations:

$$
\left\{\begin{array}{l}
\varrho_{t}^{\mathrm{d}}+\operatorname{div}\left(\bar{\rho} v^{\mathrm{d}}\right)=F^{\delta} \\
\left(\varrho^{\delta}+\bar{\rho}\right) v_{t}^{\mathrm{d}}+\nabla\left(P^{\prime}(\bar{\rho}) \varrho^{\mathrm{d}}+\lambda m N_{1}^{\mathrm{d}}\right)-\mu_{1} \Delta v^{\mathrm{d}}-\mu_{2} \nabla \operatorname{div} v^{\mathrm{d}} \\
\quad-\lambda \bar{M}^{\prime} N_{3}^{\mathrm{d}}-\lambda m \partial_{1} N^{\mathrm{d}}+\varrho g^{\mathrm{d}} e_{3}=G^{\delta} \\
N_{t}^{\mathrm{d}}=m \partial_{1} v^{\mathrm{d}}-v_{3}^{\mathrm{d}} \bar{M}^{\prime}-\bar{M} \operatorname{div} v^{\mathrm{d}}+H^{\delta} \\
\operatorname{div} N^{\mathrm{d}}=0
\end{array}\right.
$$


where

$$
\begin{aligned}
F^{\delta}:= & -\operatorname{div}\left(\varrho^{\delta} v^{\delta}\right), \\
G^{\delta}:= & \lambda N^{\delta} \cdot \nabla N^{\delta}-\nabla\left(\int_{0}^{\varrho^{\delta}}\left(\varrho^{\delta}-z\right) P^{\prime \prime}(z+\bar{\rho}) \mathrm{d} z+\lambda\left|N^{\delta}\right|^{2} / 2\right) \\
& \quad-\left(\varrho^{\delta}+\bar{\rho}\right) v^{\delta} \cdot \nabla v^{\delta}-\varrho^{\delta} v_{t}^{\mathrm{a}}, \\
H^{\delta}:= & N^{\delta} \cdot \nabla v^{\delta}-v^{\delta} \cdot \nabla N^{\delta}-N^{\delta} \operatorname{div} v^{\delta},
\end{aligned}
$$

with initial conditions

$$
\left(\varrho^{\mathrm{d}}(0), v^{\mathrm{d}}(0), N^{\mathrm{d}}(0)\right):=\left(0, \delta^{2} v_{\mathrm{r}}, 0\right)
$$

and

$$
\operatorname{div} N^{\mathrm{d}}(0)=0 .
$$

Moreover, we can deduce the following error estimate from the error equations, which plays a key role in the proof of Theorem 2.3 .

Lemma 6.2. Let $\epsilon \in(0,1)$ and $\delta \in(0, \tau) \subset(0,1)$. Denote

$$
\begin{aligned}
C_{5} & :=(1+\Lambda)\left(\left\|\left(\tilde{\varrho}_{0}, \tilde{v}_{0}, \tilde{N}_{0}\right)\right\|_{2}+2 C_{4}\right)^{2}, \\
T^{*} & :=\sup \left\{t \in\left(0, T^{\max }\right) \mid \mathcal{S}\left(\varrho^{\delta}(\tau), v^{\delta}(\tau), N^{\delta}(\tau)\right)+\left\|v_{s}^{\delta}\right\|_{L^{2}\left((0, \tau), H^{1}\right)}\right. \\
& \left.\leq 2 C_{3} C_{5} \delta e^{\Lambda \tau} \quad \text { for any } \tau \in[0, t]\right\} .
\end{aligned}
$$

If $T^{\max }=\infty$, and $\left(\varrho^{\delta}, v^{\delta}, N^{\delta}\right)$ satisfies (6.7) and

$$
\left\|\left(\varrho^{\delta}, v^{\delta}, N^{\delta}\right)(t)\right\|_{3} \leq \epsilon \text { on }[0, \infty)
$$

then there is a constant $C_{6}$, such that

$$
\mathcal{S}\left(\varrho^{\mathrm{d}}(t), v^{\mathrm{d}}(t), N^{\mathrm{d}}(t)\right)+\left\|v_{\tau}^{\mathrm{d}}\right\|_{L^{2}\left((0, t), H^{1}\right)} \leq C_{6} \sqrt{f(\epsilon, \delta, t)} \quad \text { for any } t \in\left(0, T^{*}\right],
$$

where $\mathcal{S}\left(\varrho^{\mathrm{d}}(t), v^{\mathrm{d}}(t), N^{\mathrm{d}}(t)\right)$ is defined by (6.8) and $f(\epsilon, \delta, t):=\delta^{3} e^{3 \Lambda t}+\epsilon^{\frac{2}{3}} \delta^{\frac{7}{3}} e^{\frac{7 \Lambda t}{3}}$.

Remark 6.1. It should be remarked that (6.7) automatically holds for sufficiently small $\epsilon$. In fact, by virtue of (3.3), there is a positive constant $\epsilon_{0}$, such that (6.7) holds for any $\varrho^{\delta}$ satisfying $\left\|\varrho^{\delta}\right\|_{3} \leq \epsilon_{0}$.

Proof. From Lemma 6.1 one gets

$$
\begin{aligned}
& \sqrt{1+\left\|\left(\varrho_{0}^{\delta}, v_{0}^{\delta}, N_{0}^{\delta}\right)\right\|_{1}^{2}}\left\|\left(\varrho_{0}^{\delta}, v_{0}^{\delta}, N_{0}^{\delta}\right)\right\|_{2} \\
& \leq \delta \sqrt{1+\left(\left\|\left(\varrho_{0}, \tilde{v}_{0}, \tilde{N}_{0}\right)\right\|_{2}+C_{4}\right)^{2}}\left(\left\|\left(\tilde{\varrho}_{0}, \tilde{v}_{0}, \tilde{N}_{0}\right)\right\|_{2}+C_{4}\right) \leq C_{5} \delta .
\end{aligned}
$$

Thus, in view of the regularity of $\left(\varrho^{\delta}, v^{\delta}, N^{\delta}\right)$ and the estimate (6.8), we see that $T^{*}>0$. Moreover, by the definition of $T^{*}$,

$$
\mathcal{S}\left(\varrho^{\delta}(t), v^{\delta}(t), N^{\delta}(t)\right)+\left\|v_{\tau}^{\delta}\right\|_{L^{2}\left((0, t), H^{1}\right)} \leq 2 C_{3} C_{5} \delta e^{\Lambda t} \quad \text { on }\left[0, T^{*}\right]
$$

or

$$
\mathcal{S}\left(\varrho^{\delta}\left(T^{*}\right), v^{\delta}\left(T^{*}\right), N^{\delta}\left(T^{*}\right)\right)+\left\|v_{\tau}^{\delta}\right\|_{L^{2}\left(\left(0, T^{*}\right), H^{1}\right)}=2 C_{3} C_{5} \delta e^{\Lambda T^{*}} \text { in the case of } T^{*}<\infty .
$$


We next show (6.20). By (6.17) 2 we find that

$$
\begin{aligned}
& \frac{\mathrm{d}}{\mathrm{d} t} \int\left(\varrho^{\delta}+\bar{\rho}\right)\left|v_{t}^{\mathrm{d}}\right|^{2} \mathrm{~d} x=2 \int\left(\left(\varrho^{\delta}+\bar{\rho}\right) v_{t}^{\mathrm{d}}\right)_{t} \cdot v_{t}^{\mathrm{d}} \mathrm{d} x-\int \varrho_{t}^{\delta}\left|v_{t}^{\mathrm{d}}\right|^{2} \mathrm{~d} x \\
& =2 \int\left(P^{\prime}(\bar{\rho}) \varrho_{t}^{\mathrm{d}}+\lambda m \partial_{t} N_{1}^{\mathrm{d}}\right) \operatorname{div} v_{t}^{\mathrm{d}} \mathrm{d} x+2 \int\left(\lambda \bar{M}^{\prime} \partial_{t} N_{3}^{\mathrm{d}}+\lambda m \partial_{1} N_{t}^{\mathrm{d}}-g \varrho_{t}^{\mathrm{d}} e_{3}\right) \cdot v_{t}^{\mathrm{d}} \mathrm{d} x \\
& \quad \quad+\int\left(2 G_{t}^{\delta}-\varrho_{t}^{\delta} v_{t}^{\mathrm{d}}\right) \cdot v_{t}^{\mathrm{d}} \mathrm{d} x-2 \int\left(\mu_{1}\left|\nabla v_{t}^{\mathrm{d}}\right|^{2}+\mu_{2}\left|\operatorname{div} v_{t}^{\mathrm{d}}\right|^{2}\right) \mathrm{d} x \\
& =: \tilde{R}_{1}(t)+\tilde{R}_{2}(t)+R_{3}(t)+R_{4}(t) .
\end{aligned}
$$

On the other hand, arguing similarly to that for $(\underline{3.46})-(3.48)$, we use $(\underline{6.17})_{1},(\underline{6.17})_{3},(1.5)$ to infer that the first two integrals $\tilde{R}_{1}(t)$ and $\tilde{R}_{2}(t)$ can be written as follows.

$$
\begin{aligned}
\tilde{R}_{1}(t) & +\tilde{R}_{2}(t)=2 \int\left(-P^{\prime}(\bar{\rho}) \operatorname{div}\left(\bar{\rho} v^{\mathrm{d}}\right)+\lambda m\left(m \partial_{1} v_{1}^{\mathrm{d}}-m^{\prime} v_{3}^{\mathrm{d}}-m \operatorname{div} v^{\mathrm{d}}\right)\right) \operatorname{div} v_{t}^{\mathrm{d}} \mathrm{d} x \\
& +\int\left(2 \lambda m^{2}\left(\partial_{1}^{2} v^{\mathrm{d}} \cdot v_{t}^{\mathrm{d}}-\partial_{1} \operatorname{div} v^{\mathrm{d}} \partial_{t} v_{1}^{\mathrm{d}}\right)+g \operatorname{div}\left(\bar{\rho} v^{\mathrm{d}}\right) \partial_{t} v_{3}^{\mathrm{d}}\right) \mathrm{d} x+R_{2}(t) \\
= & \frac{\mathrm{d}}{\mathrm{d} t} E\left(v^{\mathrm{d}}(t)\right)+R_{2}(t),
\end{aligned}
$$

where

$$
R_{2}(t):=2 \int F^{\delta}\left(P^{\prime}(\bar{\rho}) \operatorname{div} v_{t}^{\mathrm{d}}-g \partial_{t} v_{3}^{\mathrm{d}}\right) \mathrm{d} x+2 \lambda \int\left(m H_{1}^{\delta} \operatorname{div} v_{t}^{\mathrm{d}}+m^{\prime} H_{3}^{\delta} \partial_{t} v_{1}^{\mathrm{d}}+m \partial_{1} H^{\delta} \cdot v_{t}^{\mathrm{d}}\right) \mathrm{d} x .
$$

Thus the equality (6.23) can be rewritten as

$$
\frac{\mathrm{d}}{\mathrm{d} t} \int\left(\left(\varrho^{\delta}+\bar{\rho}\right)\left|v_{t}^{\mathrm{d}}\right|^{2}-E\left(v^{\mathrm{d}}(t)\right)\right) \mathrm{d} x+2 \int\left(\mu_{1}\left|\nabla v_{t}^{\mathrm{d}}\right|^{2}+\mu_{2}\left|\operatorname{div} v_{t}^{\mathrm{d}}\right|^{2}\right) \mathrm{d} x=R_{2}(t)+R_{3}(t) .
$$

Recalling that $v^{\mathrm{d}}(0)=\delta^{2} v_{\mathrm{r}}$, we integrate (6.24) in time from 0 to $t$ to get

$$
\left\|\sqrt{\varrho^{\delta}+\bar{\rho}} v_{t}^{\mathrm{d}}(t)\right\|_{0}^{2}+2 \int_{0}^{t}\left(\mu_{1}\left\|\nabla v_{\tau}^{\mathrm{d}}\right\|_{0}^{2}+\mu_{2}\left\|\operatorname{div} v_{\tau}^{\mathrm{d}}\right\|_{0}^{2}\right) \mathrm{d} \tau=E\left(v^{\mathrm{d}}(t)\right)+\sum_{i=1}^{3} \Re_{i}
$$

where

$$
\mathfrak{R}_{1}:=\left.\int\left(\varrho^{\delta}+\bar{\rho}\right)\left|v_{t}^{\mathrm{d}}\right|^{2} \mathrm{~d} x\right|_{t=0}-E\left(\delta^{2} v_{\mathrm{r}}\right), \quad \mathfrak{R}_{2}:=\int_{0}^{t} R_{2}(\tau) \mathrm{d} \tau, \quad \text { and } \mathfrak{R}_{3}:=\int_{0}^{t} R_{3}(\tau) \mathrm{d} \tau
$$

are bounded from below.

Multiplying (6.17) $)_{2}$ by $v_{t}^{\mathrm{d}}$ in $L^{2}$, one gets

$$
\begin{aligned}
& \int\left(\varrho^{\delta}+\bar{\rho}\right)\left|v_{t}^{\mathrm{d}}\right|^{2} \mathrm{~d} x \\
& =\int\left(\mu_{1} \Delta v^{\mathrm{d}}+\mu_{2} \nabla \operatorname{div} v^{\mathrm{d}}+\lambda \bar{M}^{\prime} N_{3}^{\mathrm{d}}+\lambda m \partial_{1} N^{\mathrm{d}}-\varrho g^{\mathrm{d}} e_{3}-\nabla\left(P^{\prime}(\bar{\rho}) \varrho^{\mathrm{d}}+\lambda m N_{1}^{\mathrm{d}}\right)+G^{\delta}\right) \cdot v_{t}^{\mathrm{d}} \mathrm{d} x .
\end{aligned}
$$

Applying (6.7) and Cauchy-Schwarz's inequality, we obtain

$$
\left\|\sqrt{\varrho^{\delta}+\bar{\rho}} v_{t}^{\mathrm{d}}\right\|_{0}^{2} \lesssim\left\|\left(\varrho^{\mathrm{d}}, N^{\mathrm{d}}\right)\right\|_{1}^{2}+\left\|v^{\mathrm{d}}\right\|_{2}^{2}+\left\|G^{\delta}\right\|_{0}^{2} .
$$


From the definition of $v_{t}^{\mathrm{a}}$ it follows that

$$
\left\|\partial_{t}^{j} v^{\mathrm{a}}\right\|_{k}=\Lambda^{j} \delta e^{\Lambda t}\left\|\tilde{v}_{0}\right\|_{k} \lesssim \Lambda^{j} \delta e^{\Lambda t} \quad \text { for } 0 \leq k, j \leq 3 .
$$

So, using (6.27), (6.21), (6.19), (6.7), the interpolation inequality (3.4), and the Nirenberg interpolation inequality (see [3, 5.8 Theorem])

$$
\|w\|_{L^{\infty}} \lesssim\|w\|_{0}^{\frac{1}{2}}\|w\|_{3}^{\frac{1}{2}}
$$

we arrive at

$$
\begin{aligned}
\left\|G^{\delta}\right\|_{0}^{2} & \lesssim\left\|v^{\delta}\right\|_{L^{\infty}}^{2}\left\|v^{\delta}\right\|_{1}^{2}+\left\|\varrho^{\delta}\right\|_{0}^{2}\left\|v_{t}^{\mathrm{a}}\right\|_{2}^{2}+\left\|\left(\varrho^{\delta}, N^{\delta}\right)\right\|_{L^{\infty}}^{2}\left\|\left(\varrho^{\delta}, N^{\delta}\right)\right\|_{1}^{2} \\
& \lesssim\left\|v^{\delta}\right\|_{0}\left\|v^{\delta}\right\|_{3}\left\|v^{\delta}\right\|_{1}^{2}+\left\|\left(\varrho^{\delta}, N^{\delta}\right)\right\|_{0}^{\frac{7}{3}}\left\|\left(\varrho^{\delta}, N^{\delta}\right)\right\|_{3}^{\frac{5}{3}}+\delta^{4} e^{4 \Lambda t} \\
& \lesssim \delta^{4} e^{4 \Lambda t}+\epsilon \delta^{3} e^{3 \Lambda t}+\epsilon^{\frac{5}{3}} \delta^{\frac{7}{3}} e^{\frac{7 \Lambda t}{3}} .
\end{aligned}
$$

Chaining the estimates (6.26) and (6.29) together and taking then to the limit as $t \rightarrow 0$, we apply Lemma 6.1 and (6.18) to obtain the following estimate on $\mathfrak{R}_{1}$ :

$$
\begin{aligned}
\mathfrak{R}_{1} & =\lim _{t \rightarrow 0}\left\|\sqrt{\varrho^{\delta}+\bar{\rho}} v_{t}^{\mathrm{d}}(t)\right\|_{0}^{2}-E\left(\delta^{2} v_{\mathrm{r}}\right) \\
& \lesssim \lim _{t \rightarrow 0}\left(\left\|\left(\varrho^{\mathrm{d}}, N^{\mathrm{d}}\right)\right\|_{1}^{2}+\left\|v^{\mathrm{d}}\right\|_{2}^{2}+\delta^{4} e^{4 \Lambda t}+\epsilon \delta^{3} e^{3 \Lambda t}+\epsilon^{\frac{5}{3}} \delta^{\frac{7}{3}} e^{\frac{7 \Lambda t}{3}}\right)+\delta^{4} \\
& \lesssim \delta^{4}+\epsilon \delta^{3}+\epsilon^{\frac{5}{3}} \delta^{\frac{7}{3}} \lesssim \delta^{3}+\epsilon^{\frac{2}{3}} \delta^{\frac{7}{3}} .
\end{aligned}
$$

To bound $\mathfrak{R}_{2}(t)$, recalling the definition of $\mathfrak{R}_{2}(t)$, we have

$$
\mathfrak{R}_{2}(t) \lesssim \int_{0}^{t}\left(\left\|F^{\delta}\right\|_{0}+\left\|H^{\delta}\right\|_{0}\right)\left\|\partial_{t} v^{\mathrm{d}}\right\|_{1} \mathrm{~d} \tau
$$

By the interpolation inequality [48, Theorem 1.49] in $L^{p}$, (3.4) in $H^{k}$ and the embedding inequality (3.2), we see that

$$
\|\nabla w\|_{L^{3}} \lesssim\|\nabla w\|_{0}^{\frac{1}{2}}\|\nabla w\|_{L^{6}}^{\frac{1}{2}} \lesssim\|w\|_{1}^{\frac{1}{2}}\|w\|_{2}^{\frac{1}{2}} \lesssim\|w\|_{0}^{\frac{1}{2}}\|w\|_{3}^{\frac{1}{2}}
$$

from which, (6.28) and the embedding inequality it follows that

$$
\begin{aligned}
\left\|F^{\delta}\right\|_{0}+\left\|H^{\delta}\right\|_{0} & \lesssim\left\|v^{\delta}\right\|_{1}\left\|\nabla\left(\varrho^{\delta}, N^{\delta}\right)\right\|_{L^{3}}+\left\|\left(\varrho^{\delta}, N^{\delta}\right)\right\|_{L^{\infty}}\left\|\nabla v^{\delta}\right\|_{0} \\
& \lesssim\left\|v^{\delta}\right\|_{1}\left\|\left(\varrho^{\delta}, N^{\delta}\right)\right\|_{0}^{\frac{1}{2}}\left\|\left(\varrho^{\delta}, N^{\delta}\right)\right\|_{3}^{\frac{1}{2}}+\left\|\left(\varrho^{\delta}, N^{\delta}\right)\right\|_{0}^{\frac{1}{2}}\left\|\left(\varrho^{\delta}, N^{\delta}\right)\right\|_{3}^{\frac{1}{2}}\left\|v^{\delta}\right\|_{1} \\
& \lesssim \epsilon^{\frac{1}{2}} \delta^{\frac{3}{2}} e^{\frac{3 \Lambda t}{2}} .
\end{aligned}
$$

Plugging (6.32) into (6.31), we make use of (6.27), (6.21) and Hölder's inequality to conclude

$$
\mathfrak{R}_{2}(t) \lesssim\left(\int_{0}^{t} \epsilon \delta^{3} e^{3 \Lambda t} \mathrm{~d} \tau\right)^{\frac{1}{2}}\left(\int_{0}^{t}\left\|v_{\tau}^{\mathrm{a}}\right\|_{1}^{2} \mathrm{~d} \tau+\int_{0}^{t}\left\|v_{\tau}^{\delta}\right\|_{1}^{2} \mathrm{~d} \tau\right)^{\frac{1}{2}} \lesssim \epsilon^{\frac{1}{2}} \delta^{\frac{5}{2}} e^{\frac{5 \Lambda t}{2}}
$$

Now, to control $\mathfrak{R}_{3}(t)$, we integrate by parts and use Hölder's inequality to find that

$$
\begin{aligned}
\mathfrak{R}_{3}(t)= & -2 \lambda \int_{0}^{t} \int\left(N_{\tau}^{\delta} \cdot \nabla v_{\tau}^{\mathrm{d}} \cdot N^{\delta}+N^{\delta} \cdot \nabla v_{\tau}^{\mathrm{d}} \cdot N_{\tau}^{\delta}-N^{\delta} \cdot N_{\tau}^{\delta} \operatorname{div} v_{\tau}^{\mathrm{d}} / 2\right) \mathrm{d} x \mathrm{~d} \tau \\
& -2 \int_{0}^{t} \int\left(\left(\varrho^{\delta}+\bar{\rho}\right) v_{t}^{\delta} \cdot \nabla v^{\delta}+\left(\varrho^{\delta}+\bar{\rho}\right) v^{\delta} \cdot \nabla v_{t}^{\delta}+\varrho^{\delta} v_{t t}^{\mathrm{a}}\right) \cdot v_{\tau}^{\mathrm{d}} \mathrm{d} x \mathrm{~d} \tau \\
& -\int_{0}^{t} \int \varrho_{\tau}^{\delta}\left(\left(v_{\tau}^{\mathrm{a}}+v_{\tau}^{\delta}+2 v^{\delta} \cdot \nabla v^{\delta}\right) \cdot v_{\tau}^{\mathrm{d}}-2 \int_{0}^{\varrho^{\delta}} P^{\prime \prime}(z+\bar{\rho}) \mathrm{d} z \operatorname{div} v_{\tau}^{\mathrm{d}}\right) \mathrm{d} x \mathrm{~d} \tau \\
= & \sum_{i=1}^{3} \mathfrak{R}_{3, i}(t),
\end{aligned}
$$


where the terms on the right hand side can be estimated as follows, employing (6.28), (6.27), (6.21) and (6.19).

$$
\begin{aligned}
\mathfrak{R}_{3,1}(t) & \lesssim \int_{0}^{t}\left\|N_{\tau}^{\delta}\right\|_{0}\left\|N^{\delta}\right\|_{\infty}\left\|v_{\tau}^{\mathrm{d}}\right\|_{1} \mathrm{~d} \tau \\
& \lesssim\left(\int_{0}^{t}\left\|N_{\tau}^{\delta}\right\|_{0}^{2}\left\|N^{\delta}\right\|_{0}\left\|N^{\delta}\right\|_{3} \mathrm{~d} \tau\right)^{\frac{1}{2}}\left(\int_{0}^{t}\left\|v_{\tau}^{\mathrm{a}}\right\|_{1}^{2} \mathrm{~d} \tau+\int_{0}^{t}\left\|v_{\tau}^{\delta}\right\|_{1}^{2} \mathrm{~d} \tau\right)^{\frac{1}{2}} \\
& \lesssim \epsilon^{\frac{1}{2}} \delta^{\frac{5}{2}} e^{\frac{5 \Lambda t}{2}}
\end{aligned}
$$

while one can utilize (6.27), (6.21), (6.7), and (3.2) to deduce that

$$
\begin{aligned}
\mathfrak{R}_{3,2}(t) & \lesssim \int_{0}^{t}\left(\left\|\varrho^{\delta}\right\|_{0}\left\|v_{\tau \tau}^{\mathrm{a}}\right\|_{1}+\left\|v^{\delta}\right\|_{1}\left\|v_{\tau}^{\delta}\right\|_{1}\right)\left\|v_{\tau}^{\mathrm{d}}\right\|_{1} \mathrm{~d} \tau \\
& \lesssim \int_{0}^{t}\left[\delta^{2} e^{2 \Lambda \tau}\left(\delta e^{\Lambda \tau}+\left\|v_{\tau}^{\delta}\right\|_{1}\right)+\delta e^{\Lambda \tau}\left\|v_{\tau}^{\delta}\right\|_{1}^{2}\right] \mathrm{d} \tau \\
& \lesssim \int_{0}^{t} \delta e^{\Lambda \tau}\left(\delta^{2} e^{2 \Lambda \tau}+\left\|v_{\tau}^{\delta}\right\|_{1}^{2}\right) \mathrm{d} \tau \lesssim \delta^{3} e^{3 \Lambda t}
\end{aligned}
$$

Finally, similar to the derivation of (6.34) and (6.35), one has

$$
\begin{aligned}
\mathfrak{R}_{3,3}(t) & \lesssim \int_{0}^{t}\left\|\varrho_{\tau}^{\delta}\right\|_{0}\left(\left\|v_{\tau}^{\mathrm{a}}\right\|_{L^{3}}+\left\|v_{\tau}^{\delta}\right\|_{L^{3}}+\left\|\varrho^{\delta}\right\|_{L^{\infty}}+\left\|v^{\delta}\right\|_{1}\left\|v^{\delta}\right\|_{2}\right)\left\|v_{\tau}^{\mathrm{d}}\right\|_{1} \mathrm{~d} \tau \\
& \lesssim \int_{0}^{t} \delta e^{\Lambda \tau}\left(\left\|\varrho^{\delta}\right\|_{0}^{\frac{1}{2}}\left\|\varrho^{\delta}\right\|_{3}^{\frac{1}{2}}\left\|v_{\tau}^{\mathrm{d}}\right\|_{1}+\delta^{2} e^{2 \Lambda \tau}+\left\|v_{\tau}^{\delta}\right\|_{1}^{2}\right) \mathrm{d} \tau \\
& \lesssim \delta^{\frac{5}{2}} e^{\frac{5 \Lambda t}{2}}\left(\epsilon^{\frac{1}{2}}+\delta^{\frac{1}{2}} e^{\frac{\Lambda t}{2}}\right)
\end{aligned}
$$

Thus, summing up the estimates (6.34)-(6.36), (6.33) and (6.30), we use Young's inequality to infer

$$
\sum_{i=1}^{3} \mathfrak{R}_{i} \lesssim \delta^{3} e^{3 \Lambda t}+\epsilon^{\frac{1}{2}} \delta^{\frac{5}{2}} e^{\frac{5 \Lambda t}{2}}+\epsilon^{\frac{2}{3}} \delta^{\frac{7}{3}} \lesssim f(\epsilon, \delta, t)
$$

Combining (6.25) with (6.37), one obtains

$$
\left\|\sqrt{\varrho^{\delta}+\bar{\rho}} v_{t}^{\mathrm{d}}(t)\right\|_{0}^{2}+2 \int_{0}^{t}\left(\mu_{1}\left\|\nabla v_{\tau}^{\mathrm{d}}\right\|_{0}^{2}+\mu_{2}\left\|\operatorname{div} v_{\tau}^{\mathrm{d}}\right\|_{0}^{2}\right) \mathrm{d} \tau \leq E\left(v^{\mathrm{d}}(t)\right)+c f(\epsilon, \delta, t) .
$$

Thanks to (6.2), we have

$$
\begin{aligned}
E\left(v^{\mathrm{d}}\right) & \leq \Lambda^{2} \int \bar{\rho}\left|v^{\mathrm{d}}\right|^{2} \mathrm{~d} x+\Lambda\left(\mu_{1}\left\|\nabla v^{\mathrm{d}}\right\|_{0}^{2}+\mu_{2}\left\|\operatorname{div} v^{\mathrm{d}}\right\|_{0}^{2}\right) \\
& =\Lambda^{2} \int\left(\varrho^{\delta}+\bar{\rho}\right)\left|v^{\mathrm{d}}\right|^{2} \mathrm{~d} x+\Lambda\left(\mu_{1}\left\|\nabla v^{\mathrm{d}}\right\|_{0}^{2}+\mu_{2}\left\|\operatorname{div} v^{\mathrm{d}}\right\|_{0}^{2}\right)-\Lambda^{2} \int \varrho^{\delta}\left|v^{\mathrm{d}}\right|^{2} \mathrm{~d} x .
\end{aligned}
$$

On the other hand,

$$
\int \varrho^{\delta}\left|v^{\mathrm{d}}\right|^{2} \mathrm{~d} x \lesssim\left\|\varrho^{\delta}\right\|_{L^{\infty}}\left\|v^{\mathrm{d}}\right\|_{0}^{2} \lesssim\left\|\varrho^{\delta}\right\|_{0}^{\frac{1}{2}}\left\|\varrho^{\delta}\right\|_{3}^{\frac{1}{2}} \delta^{2} e^{2 \Lambda t} \lesssim \epsilon^{\frac{1}{2}} \delta^{\frac{5}{2}} e^{\frac{5 \Lambda t}{2}}
$$


Combining the above three inequalities together, we arrive at

$$
\begin{aligned}
& \left\|\sqrt{\varrho^{\delta}+\bar{\rho}} v_{t}^{\mathrm{d}}(t)\right\|_{0}^{2}+2 \int_{0}^{t}\left(\mu_{1}\left\|\nabla v_{\tau}^{\mathrm{d}}\right\|_{0}^{2}+\mu_{2}\left\|\operatorname{div} v_{\tau}^{\mathrm{d}}\right\|_{0}^{2}\right) \mathrm{d} \tau \\
& \leq \Lambda^{2}\left\|\sqrt{\varrho^{\delta}+\bar{\rho}} v^{\mathrm{d}}(t)\right\|_{0}^{2}+\Lambda\left(\mu_{1}\left\|\nabla v^{\mathrm{d}}\right\|_{0}^{2}+\mu_{2}\left\|\operatorname{div} v^{\mathrm{d}}\right\|_{0}^{2}\right)+c f(\epsilon, \delta, t) .
\end{aligned}
$$

Recalling that $v^{\mathrm{d}} \in C^{0}\left(\left[0, T^{\mathrm{max}}\right), H^{3}\right)$ and $v^{\mathrm{d}}(0)=\delta^{2} v_{\mathrm{r}}$, we apply Newton-Leibniz's formula and Cauchy-Schwarz's inequality to find that

$$
\begin{aligned}
& \Lambda\left(\mu_{1}\left\|\nabla v^{\mathrm{d}}(t)\right\|_{0}^{2}+\mu_{2}\left\|\operatorname{div} v^{\mathrm{d}}(t)\right\|_{0}^{2}\right) \\
& =2 \Lambda \mu_{1} \int_{0}^{t} \int \nabla v^{\mathrm{d}}: \nabla v_{\tau}^{\mathrm{d}} \mathrm{d} x \mathrm{~d} \tau+2 \Lambda \mu_{2} \int_{0}^{t} \int \operatorname{div} v^{\mathrm{d}} \operatorname{div} v_{\tau}^{\mathrm{d}} \mathrm{d} x \mathrm{~d} \tau \\
& \quad+\delta^{4} \Lambda\left(\mu_{1}\left\|\nabla v_{\mathrm{r}}\right\|_{0}^{2}+\mu_{2}\left\|\operatorname{div} v_{\mathrm{r}}\right\|_{0}^{2}\right) \\
& \leq \Lambda^{2} \int_{0}^{t}\left(\mu_{1}\left\|\nabla v^{\mathrm{d}}\right\|_{0}^{2}+\mu_{2}\left\|\operatorname{div} v^{\mathrm{d}}\right\|_{0}^{2}\right) \mathrm{d} \tau+\int_{0}^{t}\left(\mu_{1}\left\|\nabla v_{\tau}^{\mathrm{d}}\right\|_{0}^{2}+\mu_{2}\left\|\operatorname{div} v_{\tau}^{\mathrm{d}}\right\|_{0}^{2}\right) \mathrm{d} \tau .
\end{aligned}
$$

Combining (6.38) with (6.39), one gets

$$
\begin{aligned}
& \frac{1}{\Lambda}\left\|\sqrt{\varrho^{\delta}+\bar{\rho}} v_{t}^{\mathrm{d}}(t)\right\|_{0}^{2}+\left(\mu_{1}\left\|\nabla v^{\mathrm{d}}(t)\right\|_{0}^{2}+\mu_{2}\left\|\operatorname{div} v^{\mathrm{d}}(t)\right\|_{0}^{2}\right) \\
& \leq \Lambda\left\|\sqrt{\varrho^{\delta}+\bar{\rho}} v^{\mathrm{d}}(t)\right\|_{0}^{2}+2 \Lambda \int_{0}^{t}\left(\mu_{1}\left\|\nabla v^{\mathrm{d}}\right\|_{0}^{2}+\mu_{2}\left\|\operatorname{div} v^{\mathrm{d}}\right\|_{0}^{2} \mathrm{~d} \tau\right) \mathrm{d} \tau+c f(\epsilon, \delta, t) .
\end{aligned}
$$

On the other hand,

$$
\begin{aligned}
\frac{\mathrm{d}}{\mathrm{d} t}\left\|\sqrt{\varrho^{\delta}+\bar{\rho}} v^{\mathrm{d}}\right\|_{0}^{2} & =2 \int\left(\varrho^{\delta}+\bar{\rho}\right) v^{\mathrm{d}} \cdot v_{t}^{\mathrm{d}} \mathrm{d} x+\int \varrho_{t}^{\delta}\left|v^{\mathrm{d}}\right|^{2} \mathrm{~d} x \\
& \leq \frac{1}{\Lambda}\left\|\sqrt{\left(\varrho^{\delta}+\bar{\rho}\right)} v_{t}^{\mathrm{d}}\right\|_{0}^{2}+\Lambda\left\|\sqrt{\varrho^{\delta}+\bar{\rho}} v^{\mathrm{d}}\right\|_{0}^{2}+\int \varrho_{t}^{\delta}\left|v^{\mathrm{d}}\right|^{2} \mathrm{~d} x
\end{aligned}
$$

and

$$
\int \varrho_{t}^{\delta}\left|v^{\mathrm{d}}\right|^{2} \mathrm{~d} x=-\int \operatorname{div}\left(\bar{\rho} v^{\delta}+\varrho^{\delta} v^{\delta}\right)\left|v^{\mathrm{d}}\right|^{2} \mathrm{~d} x \lesssim\left(\left\|v^{\delta}\right\|_{1}+\left\|\varrho^{\delta}\right\|_{2}\left\|v^{\delta}\right\|_{1}\right)\left\|v^{\mathrm{d}}\right\|_{1}^{2} \lesssim \delta^{3} e^{3 \Lambda t}
$$

If we put the previous three estimates together, we get the differential inequality

$$
\begin{aligned}
& \frac{\mathrm{d}}{\mathrm{d} t}\left\|\sqrt{\varrho^{\delta}+\bar{\rho}} v^{\mathrm{d}}(t)\right\|_{0}^{2}+\left(\mu_{1}\left\|\nabla v^{\mathrm{d}}(t)\right\|_{0}^{2}+\mu_{2}\left\|\operatorname{div} v^{\mathrm{d}}(t)\right\|_{0}^{2}\right) \\
& \leq 2 \Lambda\left(\left\|\sqrt{\varrho^{\delta}+\bar{\rho}} v^{\mathrm{d}}(t)\right\|_{0}^{2}+\int_{0}^{t}\left(\mu_{1}\left\|\nabla v^{\mathrm{d}}\right\|_{0}^{2}+\mu_{2}\left\|\operatorname{div} v^{\mathrm{d}}\right\|_{0}^{2}\right) \mathrm{d} \tau\right)+c f(\epsilon, \delta, t) .
\end{aligned}
$$

Recalling $v^{\mathrm{d}}(0)=\delta^{2} v_{\mathrm{r}}$, one can apply Gronwall's inequality to (6.41) to conclude

$$
\begin{array}{ll}
\| & \sqrt{\varrho^{\delta}+\bar{\rho}} v^{\mathrm{d}}(t)\left\|_{0}^{2}+\mu_{1} \int_{0}^{t}\right\| \nabla v^{\mathrm{d}}\left\|_{0}^{2} \mathrm{~d} \tau+\mu_{2} \int_{0}^{t}\right\| \operatorname{div} v^{\mathrm{d}} \|_{0}^{2} \mathrm{~d} \tau \\
& \leq e^{2 \Lambda t}\left(\int_{0}^{t} c f(\epsilon, \delta, \tau) e^{-2 \Lambda \tau} \mathrm{d} \tau+\delta^{4}\left\|\sqrt{\varrho_{0}^{\delta}+\bar{\rho}} v^{\mathrm{r}}\right\|_{0}^{2}\right) \lesssim f(\epsilon, \delta, t), \quad \forall t \in\left(0, T^{*}\right) .
\end{array}
$$

Moreover, using (3.6) and (6.7), we can further deduce from (6.42), (6.40) and (6.38) that

$$
\left\|v^{\mathrm{d}}(t)\right\|_{1}^{2}+\left\|v_{t}^{\mathrm{d}}(t)\right\|_{0}^{2}+\left\|v_{\tau}^{\mathrm{d}}\right\|_{L^{2}\left((0, t), H^{1}\right)}^{2} \lesssim f(\epsilon, \delta, t) .
$$


Next we turn to the derivation of the error estimates for the perturbation density and magnetic field. It follows from the equations $(\underline{6.17})_{1}$ that

$$
\begin{aligned}
\frac{1}{2} \frac{\mathrm{d}}{\mathrm{d} t}\left\|\varrho^{\mathrm{d}}(t)\right\|_{0}^{2} & \left.=-\int \operatorname{div}\left(\bar{\rho} v^{\mathrm{d}}+\varrho^{\delta} v^{\delta}\right)\right) \varrho^{\mathrm{d}} \mathrm{d} x \\
& =-\int\left(\operatorname{div}\left(\bar{\rho} v^{\mathrm{d}}+\varrho^{\mathrm{a}} v^{\delta}\right)+\varrho^{\mathrm{d}} \operatorname{div} v^{\delta} / 2\right) \varrho^{\mathrm{d}} \mathrm{d} x \\
& \lesssim\left(\left\|v^{\mathrm{d}}\right\|_{1}+\left\|\varrho^{\mathrm{a}}\right\|_{2}\left\|v^{\delta}\right\|_{1}+\left\|\varrho^{\mathrm{d}}\right\|_{L^{\infty}}\left\|v^{\delta}\right\|_{1}\right)\left\|\varrho^{\mathrm{d}}\right\|_{0}
\end{aligned}
$$

Therefore, from (6.43), (6.28), (6.21) and (6.19), it follows that

$$
\left\|\varrho^{\mathrm{d}}(t)\right\|_{0} \lesssim \int_{0}^{t}\left(\left\|v^{\mathrm{d}}\right\|_{1}+\left\|\varrho^{\mathrm{a}}\right\|_{2}\left\|v^{\delta}\right\|_{1}+\left\|\varrho^{\mathrm{d}}\right\|_{0}^{\frac{1}{2}}\left\|\varrho^{\mathrm{d}}\right\|_{3}^{\frac{1}{2}}\left\|v^{\delta}\right\|_{1}\right) \mathrm{d} \tau \lesssim \sqrt{f(\epsilon, \delta, t)}
$$

Using (6.17) 1 again, we can argue analogously to (6.32) to deduce

$$
\left\|\varrho_{t}^{\mathrm{d}}\right\|_{0}^{2}=\left\|\operatorname{div}\left(\bar{\rho} v^{\mathrm{d}}+\varrho^{\delta} v^{\delta}\right)\right\|_{0}^{2} \lesssim f(\epsilon, \delta, t) .
$$

Similarly to (6.44) and (6.45), we get from $(6.17)_{3}$ that

$$
\left\|N^{\mathrm{d}}(t)\right\|_{0}+\left\|N_{t}^{\mathrm{d}}\right\|_{0} \lesssim \sqrt{f(\epsilon, \delta, t)} .
$$

Now, (6.20) follows from the estimates (6.43)-(6.46) immediately. This completes the proof of Lemma 6.2.

Finally, let

$$
T^{\delta}:=\frac{1}{\Lambda} \ln \frac{\epsilon}{\delta}, \quad \text { i.e., } \quad \epsilon=\delta e^{\Lambda T^{\delta}}
$$

where

$$
\epsilon:=\min \left\{\epsilon_{0}, \frac{\left(C_{3} C_{5}\right)^{2}}{4 C_{6}^{2}}, \frac{\left\|\tilde{v}_{0}\right\|_{0}^{2}}{16 C_{6}^{2}}, 1\right\}>0,
$$

and $\epsilon_{0}$ is the same as in Remark 6.1. Then we have the following conclusion concerning the relation between $T^{\delta}$ and $T^{*}$.

Lemma 6.3. Under the assumptions of Lemma 6.2, $T^{\delta}<T^{*}$.

Proof. It suffices to show that $T^{*}<\infty$, and we prove it by contradiction. Suppose that $T^{\delta} \geq T^{*}$. By virtue of the definitions of $\left(\varrho^{\mathrm{a}}, v^{\mathrm{a}}, N^{\mathrm{a}}\right), C_{5}, f(\epsilon, \delta, t)$ and $\epsilon$ in (6.47), it is easy to verify that

$$
\begin{aligned}
& \mathcal{S}\left(\varrho^{\mathrm{a}}(t), v^{\mathrm{a}}(t), N^{\mathrm{a}}(t)\right)+\left\|v_{\tau}^{\mathrm{a}}\right\|_{L^{2}\left((0, t), H^{1}\right)} \\
& \leq\left(\sqrt{\left\|\tilde{v}_{0}\right\|_{1}^{2}+\left\|\left(\tilde{\varrho}_{0}, \tilde{N}_{0}, \Lambda \tilde{\varrho}_{0}, \Lambda \tilde{v}_{0}, \Lambda \tilde{N}_{0}\right)\right\|_{0}^{2}}+\sqrt{\frac{\Lambda}{2}}\left\|\tilde{v}_{0}\right\|_{1}\right) \delta e^{\Lambda t} \\
& \leq 2 \sqrt{(1+\Lambda)}\left\|\left(\tilde{\varrho}_{0}, \tilde{v}_{0}, \tilde{N}_{0}\right)\right\|_{1} \leq C_{5} \delta e^{\Lambda t} \leq C_{3} C_{5} \delta e^{\Lambda t}
\end{aligned}
$$

and

$$
f(\epsilon, \delta, t) \leq 2 \epsilon \delta^{2} e^{2 \Lambda t} \text { for any } t \leq T^{*}
$$

Consequently,

$$
\begin{aligned}
& \left.\mathcal{S}\left(\varrho^{\delta}\left(T^{*}\right), v^{\delta}\left(T^{*}\right), N^{\delta}\left(T^{*}\right)\right)+\left\|v_{\tau}^{\delta}\right\|_{L^{2}\left(\left(0, T^{*}\right), H^{1}\right.}\right) \\
& \left.\leq \mathcal{S}\left(\varrho^{\mathrm{a}}\left(T^{*}\right), v^{\mathrm{a}}\left(T^{*}\right), N^{\mathrm{a}}\left(T^{*}\right)\right)+\left\|v_{\tau}^{\mathrm{a}}\right\|_{L^{2}\left(\left(0, T^{*}\right), H^{1}\right)}+\mathcal{S}\left(\varrho^{\mathrm{d}}\left(T^{*}\right), v^{\mathrm{d}}\left(T^{*}\right), N^{\mathrm{d}}\left(T^{*}\right)\right)+\left\|v_{\tau}^{\mathrm{d}}\right\|_{L^{2}\left(\left(0, T^{*}\right), H^{1}\right.}\right) \\
& \leq C_{3} C_{5} \delta e^{\Lambda T^{*}}+C_{6} \sqrt{f\left(\epsilon, \delta, T^{*}\right)}<\delta e^{\Lambda T^{*}}\left(C_{3} C_{5}+2 C_{6} \sqrt{\epsilon}\right)<2 C_{3} C_{5} \delta e^{\Lambda T^{*}}
\end{aligned}
$$

which contradicts (6.22). Hence, $T^{\delta}<T^{*}$. The proof is complete. 
Now we are in a position to show Theorem 2.3. Let $\epsilon$ be given by (6.48), and

$$
\varepsilon:=\epsilon \min \left\{1,\left\|\tilde{v}_{0}\right\|_{0} / 2\right\}
$$

then $\varepsilon>0$ by $(6.3)$ and the fact $\tilde{v}_{0}=\tilde{u}$. For any given $\delta \in(0, \iota)$,

(1) if the solution of the original perturbation equations $\left(\varrho^{\delta}, v^{\delta}, N^{\delta}\right)$ satisfies

$$
\left\|\left(\varrho^{\delta}, v^{\delta}, N^{\delta}\right)(T)\right\|_{3}>\epsilon \quad \text { for some } T \in\left(0, T^{\max }\right)
$$

then Theorem 2.3 automatically holds by virtue of $\epsilon \geq \varepsilon$.

(2) if (6.50) fails, then one has $T^{\max }=\infty$ by Proposition 6.2 and Remark 6.1, and

$$
\left\|\left(\varrho^{\delta}, v^{\delta}, N^{\delta}\right)(t)\right\|_{3} \leq \epsilon \quad \text { for any } t>0
$$

Thus, we can use (6.47)-(6.48) and Lemmas 6.2 6.3 to deduce that

$$
\begin{aligned}
\left\|v^{\delta}\left(T^{\delta}\right)\right\|_{0} & \geq\left\|v^{\mathrm{a}}\left(T^{\delta}\right)\right\|_{0}-\left\|v^{\mathrm{d}}\left(T^{\delta}\right)\right\|_{0}=\delta\left\|v^{\mathrm{l}}\left(T^{\delta}\right)\right\|_{0}-\left\|v^{\mathrm{d}}\left(T^{\delta}\right)\right\|_{0} \\
& >\left\|\tilde{v}_{0}\right\|_{0} \delta e^{\Lambda T^{\delta}}-2 C_{6} \sqrt{\epsilon} \delta e^{\Lambda T^{\delta}} \\
& =\left(\left\|\tilde{v}_{0}\right\|_{0}-2 C_{6} \sqrt{\epsilon}\right) \epsilon>\epsilon\left\|\bar{v}_{0}\right\|_{0} / 2 \geq \varepsilon
\end{aligned}
$$

which proves Theorem 2.3 .

\section{Positivity of the energy functional}

This section is devoted to verification of $\Xi>0$ under the assumptions of Theorem 2.4 2.5. For this purpose, we first (Fourier) transform the energy functional $E$ to a new energy functional with frequency. It should be pointed out that in the $2 \mathrm{D}$ case, $E$ is defined by

$$
E(w):=\int g \bar{\rho}^{\prime} w_{2}^{2} \mathrm{~d} x+\int 2 g \bar{\rho} w_{2} \operatorname{div} w \mathrm{~d} x-\int\left(\gamma \bar{P}|\operatorname{div} w|^{2}+\lambda m^{2}\left(\left|\partial_{1} w_{2}\right|^{2}+\left|\partial_{2} w_{2}\right|^{2}\right)\right) \mathrm{d} x
$$

where $\bar{\rho}:=\bar{\rho}\left(x_{2}\right), \operatorname{div} w=\partial_{1} w_{1}+\partial_{2} w_{2}, w=\left(w_{1}, w_{2}\right)$ and $x=\left(x_{1}, x_{2}\right)$.

We fix a spatial frequency $\xi=\left(\xi_{1}, \xi_{2}\right) \in \mathbb{R}^{2}$, and define the new unknowns

$$
\tilde{u}_{1}(x)=-\mathrm{i} \varphi\left(x_{3}\right) e^{\mathrm{i} x_{h} \cdot \xi}, \quad \tilde{u}_{2}(x)=-\mathrm{i} \theta\left(x_{3}\right) e^{\mathrm{i} x_{h} \cdot \xi}, \quad \tilde{u}_{3}(x)=\psi\left(x_{3}\right) e^{\mathrm{i} x_{h} \cdot \xi},
$$

where $\mathrm{i}^{2}=-1$. Substituting the above ansatz into (6.1), we see that $\varphi, \theta, \psi$ and $\Lambda$ satisfy the following system of ODEs:

$$
\left\{\begin{array}{l}
\Lambda^{2} \bar{\rho} \varphi+\xi_{1} P^{\prime}(\bar{\rho}) \bar{\rho}\left(\xi_{1} \varphi+\xi_{2} \theta+\psi^{\prime}\right)+\Lambda \mu_{1}\left(|\xi|^{2} \varphi-\varphi^{\prime \prime}\right)=\xi_{1} g \bar{\rho} \psi-\Lambda \mu_{2} \xi_{1}\left(\xi_{1} \varphi+\xi_{2} \theta+\psi^{\prime}\right) \\
\Lambda^{2} \bar{\rho} \theta+\xi_{2} P^{\prime}(\bar{\rho}) \bar{\rho}\left(\xi_{1} \varphi+\xi_{2} \theta+\psi^{\prime}\right)+\Lambda \mu_{1}\left(|\xi|^{2} \theta-\theta^{\prime \prime}\right) \\
\quad=\xi_{2} g \bar{\rho} \psi-\Lambda \mu_{2} \xi_{2}\left(\xi_{1} \varphi+\xi_{2} \theta+\psi^{\prime}\right)-\lambda m^{2}|\xi|^{2} \theta-\lambda m^{2} \xi_{2} \psi^{\prime} \\
\Lambda^{2} \bar{\rho} \psi-\left(\lambda m^{2}\left(\xi_{2} \theta+\psi^{\prime}\right)+P^{\prime}(\bar{\rho}) \bar{\rho}\left(\xi_{1} \varphi+\xi_{2} \theta+\psi^{\prime}\right)\right)^{\prime}+\Lambda \mu_{1}\left(|\xi|^{2} \psi-\psi^{\prime \prime}\right) \\
\quad=g \bar{\rho}\left(\xi_{1} \varphi+\xi_{2} \theta\right)-\Lambda \mu_{2}\left(\left(\xi_{1} \varphi+\xi_{2} \theta\right)^{\prime}+\psi^{\prime \prime}\right)-\lambda m^{2}\left|\xi_{1}\right|^{2} \psi
\end{array}\right.
$$

with

$$
\varphi(-l)=\theta(-l)=\psi(-l)=\varphi(l)=\theta(l)=\psi(l)=0
$$


We multiply (7.1) with $(\varphi, \theta, \psi)$ in $L^{2}(-l, l)$ respectively to deduce that

$$
\begin{aligned}
\Lambda^{2} \int_{-l}^{l} \bar{\rho}\left(\varphi^{2}+\theta^{2}+\psi^{2}\right) \mathrm{d} x_{3}= & \tilde{E}(\varphi, \theta, \psi)-\Lambda \mu_{2} \int_{-l}^{l}\left(\xi_{1} \varphi+\xi_{2} \theta-\psi^{\prime}\right)^{2} \mathrm{~d} x_{3} \\
& -\Lambda \mu_{1} \int_{-l}^{l}\left(|\xi|^{2}\left(\varphi^{2}+\theta^{2}+\psi^{2}\right)+\left|\varphi^{\prime}\right|^{2}+\left|\theta^{\prime}\right|^{2}+\left|\psi^{\prime}\right|^{2}\right) \mathrm{d} x_{3},
\end{aligned}
$$

where

$$
\begin{aligned}
& \tilde{E}(\varphi, \theta, \psi):=\int_{-l}^{l}\left(g \bar{\rho}^{\prime} \psi^{2}+2 g \bar{\rho} \psi\left(\xi_{1} \varphi+\xi_{2} \theta+\psi^{\prime}\right)\right. \\
&\left.\quad-\gamma \bar{P}\left(\xi_{1} \varphi+\xi_{2} \theta+\psi^{\prime}\right)^{2}-\lambda m^{2}\left(\xi_{1}^{2}\left(\theta^{2}+\psi^{2}\right)+\left(\xi_{2} \theta+\psi^{\prime}\right)^{2}\right)\right) \mathrm{d} x_{3},
\end{aligned}
$$

which we call the energy functional with frequency. The next lemma gives the positivity of $E$ based on the $\tilde{E}$.

Lemma 7.1. Under the assumptions of Theorem 2.4, there is a function $w_{0} \in H_{0}^{1}$, such that

$$
E\left(w_{0}\right)>0 .
$$

Proof. (1) We show (7.2) under Schwarzschild's condition. To this end, we take $\xi_{1}=L_{1}^{-1}$ and $\xi_{2}=L_{2}^{-1}$, then we can rewrite $\tilde{E}$ as follows.

$$
\begin{aligned}
\tilde{E}(\varphi, \theta, \psi):=\int_{-l}^{l}( & \left(\frac{g^{2} \bar{\rho}^{2}}{\gamma \bar{P}}+g \bar{\rho}^{\prime}-\lambda \xi_{1}^{2} m^{2}\right) \psi^{2}-\frac{\lambda \xi_{1}^{2} m^{2}}{|\xi|^{2}}\left|\psi^{\prime}\right|^{2} \\
& \left.-\gamma \bar{P}\left(\xi_{1} \varphi+\xi_{2} \theta+\psi^{\prime}-\frac{g \bar{\rho} \psi}{\gamma \bar{P}}\right)^{2}-\lambda|\xi|^{2} m^{2}\left(\theta+\frac{\xi_{2} \psi^{\prime}}{|\xi|^{2}}\right)^{2}\right) \mathrm{d} x_{3} .
\end{aligned}
$$

Recalling the definition of $\xi_{3 D}$ and the fact $\xi_{1}<\xi_{3 D}$, we see that there is a function $\psi_{0} \in$ $H_{0}^{1}(-l, l)$, such that

$$
\int_{-l}^{l}\left(\left(\frac{g^{2} \bar{\rho}^{2}}{\gamma \bar{P}}+g \bar{\rho}^{\prime}-\lambda \xi_{1}^{2} m^{2}\right) \psi_{0}^{2}-\frac{\lambda \xi_{1}^{2} m^{2}}{|\xi|^{2}}\left|\psi_{0}^{\prime}\right|^{2}\right) \mathrm{d} x_{3}>0 .
$$

Denoting

$$
\theta_{0}:=-\frac{\xi_{2} \psi_{0}^{\prime}}{|\xi|^{2}} \text { and } \varphi_{0}:=\xi_{1}^{-1}\left(\frac{g \bar{\rho} \psi_{0}}{\gamma \bar{P}}-\psi_{0}^{\prime}-\xi_{2} \theta_{0}\right)
$$

(7.3) thus reduces to

$$
\tilde{E}\left(\varphi_{0}, \theta_{0}, \psi_{0}\right)=\int_{-l}^{l}\left(\left(\frac{g^{2} \bar{\rho}^{2}}{\gamma \bar{P}}+g \bar{\rho}^{\prime}-\lambda \xi_{1}^{2} m^{2}\right) \psi_{0}^{2}-\frac{\lambda \xi_{1}^{2} m^{2}}{|\xi|^{2}}\left|\psi_{0}^{\prime}\right|^{2}\right) \mathrm{d} x_{3}>0 .
$$

We mention that Newcomb regarded (1.7) as an instability condition based the fact (7.4) [47].

Now, define

$$
w_{1}^{0}:=\varphi_{0} \sin \left(\xi \cdot x_{h}\right), \quad w_{2}^{0}:=\theta_{0} \sin \left(\xi \cdot x_{h}\right), \quad w_{3}^{0}:=\psi_{0} \cos \left(\xi \cdot x_{h}\right) .
$$

By (7.4) and the fact

$$
\int_{\mathcal{T}} \sin ^{2}\left(\xi \cdot x_{h}\right) \mathrm{d} x_{h}=\int_{\mathcal{T}} \cos ^{2}\left(\xi \cdot x_{h}\right) \mathrm{d} x_{h}=2 \pi^{2} L_{1} L_{2}>0,
$$


we have

$$
E\left(w_{0}\right)=\tilde{E}\left(\varphi_{0}, \theta_{0}, \psi_{0}\right) \int_{\mathcal{T}} \cos ^{2}\left(\xi \cdot x_{h}\right) \mathrm{d} x_{h}>0 \quad\left(w_{0}:=\left(w_{1}^{0}, w_{2}^{0}, w_{3}^{0}\right)\right),
$$

from which (7.2) follows.

(2) We proceed to show (17.2) under Tserkovnikov's condition (1.8). Taking $\xi_{1}=0$ and $\xi_{2}=1 / L_{2}$, one has

$$
\tilde{E}(\varphi, \theta, \psi)=\int_{-l}^{l}\left(\left(g \bar{\rho}^{\prime}+\frac{g^{2} \bar{\rho}^{2}}{\gamma \bar{P}+\lambda m^{2}}\right) \psi^{2}-\left(\gamma \bar{P}+\lambda m^{2}\right)\left(\xi_{2} \theta+\psi^{\prime}-\frac{g \bar{\rho} \psi}{\gamma \bar{P}+\lambda m^{2}}\right)^{2}\right) \mathrm{d} x_{3} .
$$

Recalling Tserkovnikov's condition, we find that there is a function $\psi_{0} \in H_{0}^{1}(-l, l)$, such that

$$
\int_{-l}^{l}\left(g \bar{\rho}^{\prime}+\frac{g^{2} \bar{\rho}^{2}}{\gamma \bar{P}+\lambda m^{2}}\right) \psi_{0}^{2} \mathrm{~d} x_{3}>0
$$

Denoting

$$
\theta_{0}:=\xi_{2}^{-1}\left(\frac{g \bar{\rho} \psi_{0}}{\gamma \bar{P}+\lambda m^{2}}-\psi_{0}^{\prime}\right)
$$

we easily see that for any $\varphi$,

$$
\tilde{E}\left(\varphi, \theta_{0}, \psi_{0}\right)>0
$$

Denoting $w_{0}=\left(w_{1}^{0}, w_{2}^{0}, w_{3}^{0}\right):=\left(1, \theta_{0} \sin \left(\xi_{2} x_{2}\right), \psi_{0} \cos \left(\xi_{2} x_{2}\right)\right)$, we use (7.5) and the fact

$$
\int_{\mathcal{T}} \cos ^{2}\left(\xi_{2} x_{2}\right) \mathrm{d} x_{h}=2 \pi^{2} L_{1} L_{2}>0
$$

to find that

$$
E\left(w_{0}\right)=\tilde{E}\left(\varphi, \theta_{0}, \psi_{0}\right) \int_{\mathcal{T}} \cos ^{2}\left(\xi_{2} x_{2}\right) \mathrm{d} x_{h}>0
$$

which proves the lemma.

Lemma 7.2. Under the assumptions of Theorem 2.5, there is a function $w_{0} \in H^{1}$, such that $E\left(w_{0}\right)>0$.

Proof. In the 2D case, $\tilde{E}$ is defined by

$$
\tilde{E}(\varphi, \psi):=\int_{-l}^{l}\left(g \bar{\rho}^{\prime} \psi^{2}+2 g \bar{\rho} \psi\left(\xi_{1} \varphi+\psi^{\prime}\right)-\gamma \bar{P}\left(\xi_{1} \varphi+\psi^{\prime}\right)^{2}-\lambda m^{2}\left(\xi_{1}^{2} \psi^{2}+\left|\psi^{\prime}\right|^{2}\right)\right) \mathrm{d} x_{2},
$$

which can be rewritten as

$$
\tilde{E}(\varphi, \psi):=\int_{-l}^{l}\left(\left(\frac{g^{2} \bar{\rho}^{2}}{\gamma \bar{P}}+g \bar{\rho}^{\prime}-\lambda \xi_{1}^{2} m^{2}\right) \psi^{2}-\lambda m^{2}\left|\psi^{\prime}\right|^{2}-\gamma \bar{P}\left(\xi_{1} \varphi+\psi^{\prime}-\frac{g \bar{\rho} \psi}{\gamma \bar{P}}\right)^{2}\right) \mathrm{d} x_{2} .
$$

Taking $\xi_{1}=L_{1}^{-1}$, and taking into account the definition of $\xi_{2 D}$ and the assumption $L_{1}^{-1}<\xi_{2 D}$, one sees that there is a function $\psi_{0}$ satisfying

$$
\int_{-l}^{l}\left(\left(\frac{g^{2} \bar{\rho}^{2}}{\gamma \bar{P}}+g \bar{\rho}^{\prime}-\lambda \xi_{1}^{2} m^{2}\right) \psi_{0}^{2}-\lambda m^{2}\left|\psi_{0}^{\prime}\right|^{2}\right) \mathrm{d} x_{2}>0 .
$$


Denoting

$$
\varphi_{0}:=\xi_{1}^{-1}\left(\frac{g \bar{\rho} \psi_{0}}{\gamma \bar{P}}-\psi_{0}^{\prime}\right)
$$

one has

$$
\tilde{E}\left(\varphi_{0}, \psi_{0}\right)=\int_{-l}^{l}\left(\left(\frac{g^{2} \bar{\rho}^{2}}{\gamma \bar{P}}+g \bar{\rho}^{\prime}-\lambda \xi_{1}^{2} m^{2}\right) \psi_{0}^{2}-\lambda m^{2}\left|\psi_{0}^{\prime}\right|^{2}\right) \mathrm{d} x_{2}>0 .
$$

Now, if we denote $w_{1}^{0}:=\varphi_{0} \sin \left(\xi_{1} x_{1}\right)$ and $w_{2}^{0}:=\psi_{0} \cos \left(\xi_{1} x_{1}\right)$, then we have by (7.4) and the fact

$$
\int_{2 \pi L_{1} \mathbb{T}} \sin ^{2}\left(\xi_{1} x_{1}\right) \mathrm{d} x_{1}=\int_{2 \pi L_{1} \mathbb{T}} \cos ^{2}\left(\xi_{1} x_{1}\right) \mathrm{d} x_{1}=\pi L_{1}>0
$$

that

$$
E\left(w_{0}\right)=\tilde{E}\left(\varphi_{0}, \psi_{0}\right) \int_{2 \pi L_{1} \mathbb{T}} \cos ^{2}\left(\xi_{1} x_{1}\right) \mathrm{d} x_{1}>0 \quad\left(w_{0}:=\left(w_{1}^{0}, w_{2}^{0}\right)\right)
$$

which proves the lemma.

In view of Lemmas 7.1 and 7.2 , we conclude $\Xi>0$ under the assumptions of Theorem $2.4 \sqrt{2.5}$. Therefore, we obtain Theorems 2.4 and 2.5 by following the proof of Theorem 2.3 ,

Acknowledgements. The research of Fei Jiang was supported by NSFC (Grant Nos 11471134 and 11671086) and the NSF of Fujian Province of China (Grant No. 2016J06001), and the research of Song Jiang by the National Basic Research Program (Grant No 2014CB745002) and NSFC (Grant Nos 11371065 and 11631008).

\section{References}

[1] H. Abidi, P. Zhang, On the global solution of a 3-D MHD system with initial data near equilibrium, Comm. Pure Appl. Math. 70(8) (2017) 1509-1561.

[2] D.J. Acheson, Instability by magnetic buoyancy, Solar Phys. 62(1) (1979) 23-50.

[3] R.A. Adams, J. John, Sobolev Space, Academic Press: New York, 2005.

[4] A.J. Barker, L.J. Silvers, M.R. Proctor, N.O. Weiss, Magnetic buoyancy instabilities in the presence of magnetic flux pumping at the base of the solar convection zone, Monthly Notices of the Royal Astronomical Society 424 (2012) 115-127.

[5] I.B. Bernstein, E.A. Frieman, M.D. Kruskal, R.M. Kulsrud, An energy principle for hydromagnetic stability problems, Proc. Roy. Soc. (London) A244 (1958) 17-40.

[6] G.D. Chagelishvili, J.G. Lominadze, A.D. Rogava, Fast variation of Cygnus X-1 and related sources, Astrophys. J. 347(2) (1989) 1100-1106.

[7] Y. Cho, H.J. Choe, H. Kim, Unique solvability of the initial boundary value problems for compressible viscous fluids, J. Math. Pures Appl. 83 (2004) 243-275.

[8] W. Chou, R. Matsumoto, T. Tajima, M. Umekawa, K. Shibata, Dynamics of the Parker-Jeans instability in a galactic gaseous disk, Astrophys. J. 538(2) (2000) 710-727.

[9] T.G. Cowling, Magnetohydrodynamics, volume 4, Interscience Publishers New York, 1957.

[10] S. Friedlander, P. Nataša, V. Vicol, Nonlinear instability for the critically dissipative quasigeostrophic equation, Commun. Math. Phys. 292 (2009) 797-810.

[11] S. Friedlander, W. Strauss, M. Vishik, Nonlinear instability in an ideal fluid, Annales de l'Institut Henri Poincare (C) Non Linear Analysis 14 (1997) 187-209.

[12] S. Friedlander, M. Vishik, Nonlinear instability in two dimensional ideal fluids: the case of a dominant eigenvalue, Comm. Math. Phys. 243 (2003) 261-273.

[13] Y. Fukui, et. al., Molecular loops in the galactic center: evidence for magnetic flotation, Science 314 (2006) 106-109. 
[14] Y. Guo, C. Hallstrom, D. Spirn, Dynamics near unstable, interfacial fluids, Commun. Math. Phys. 270 (2007) 635-689.

[15] Y. Guo, W. Strauss, Instability of periodic BGK equilibria, Comm. Pure Appl. Math. 48 (1995) 861-894.

[16] Y. Guo, W. Strauss, Nonlinear instability of double-humped equilibria, Ann Inst. H. Poincare Anal. Non Linéaire 12 (1995) 339-352.

[17] Y. Guo, I. Tice, Almost exponential decay of periodic viscous surface waves without surface tension, Arch. Ration. Mech. Anal. 207 (2013) 459-531.

[18] Y. Guo, I. Tice, Decay of viscous surface waves without surface tension in horizontally infinite domains , Anal. PDE 6 (2013) 1429-1533.

[19] Y. Guo, I. Tice, Local well-posedness of the viscous surface wave problem without surface tension, Anal. PDE 6 (2013) 287-369.

[20] T. Horiuchi, R. Matsumoto, Parker instability in nonuniform gravitational fields. I. Linear stability analysis, Publ. Astron. Soc. Japan 40 (1988) 147-169.

[21] X.P. Hu, Global existence for two dimensional compressible magnetohydrodynamic flows with zero magnetic diffusivity, arXiv:1405.0274v1 [math.AP] 1 May 2014 (2014).

[22] X.P. Hu, F.H. Lin, Global existence for two dimensional incompressible magnetohydrodynamic flows with zero magnetic diffusivity, arXiv:1405.0082v1 [math.AP] 1 May 2014 (2014).

[23] H.J. Hwang, Y. Guo, On the dynamical Rayleigh-Taylor instability, Arch. Rational Mech. Anal. 167 (2003) 235-253.

[24] H. Isobe, T. Miyagoshi, K. Shibata, T. Yokoyama, Filamentary structure on the Sun from the magnetic Rayleigh-Taylor instability, Nature 434(7032) (2005) 478-81.

[25] F. Jiang, S. Jiang, On instability and stability of three-dimensional gravity flows in a bounded domain, Adv. Math. 264 (2014) 831-863.

[26] F. Jiang, S. Jiang, On magnetic inhibition theory in non-resistive magnetohydrodynamic fluids, arXiv:1803.00307v2 (2018).

[27] F. Jiang, S. Jiang, On linear instability and stability of the Rayleigh-Taylor problem in magnetohydrodynamics, J. Math. Fluid Mech. 17 (2015) 639-668.

[28] F. Jiang, S. Jiang, On the stabilizing effect of the magnetic field in the magnetic Rayleigh-Taylor problem, SIAM J. Math. Aanal. 50(1) (2016) 491-540.

[29] F. Jiang, S. Jiang, W.W. Wang, Nonlinear Rayleigh-Taylor instability in nonhomogeneous incompressible viscous magnetohydrodynamic fluids, Discrete Contin. Dyn. Syst.-S 9(6) (2016) 18531898.

[30] F. Jiang, S. Jiang, Y.J. Wang, On the Rayleigh-Taylor instability for the incompressible viscous magnetohydrodynamic equations, Comm. Partial Differential Equations 39 (2014) 399-438.

[31] Y. Kagei, Large time behavior of solutions to the compressible Navier-Stokes equation in an infinite layer, Hiroshima Math. J. 38 (2008) 950-124.

[32] H. Kamaya, T. Horiuchi, R. Matsumoto, T. Hanawa, K. Shibata, S. Mineshige, Parker instability in nonuniform gravitational fields. III. the effect of a corona, Astrophys. J. 486(1) (1997) 307-315.

[33] I. Khalzov, B.P. Brown, N. Katz, C.B. Forest, Modeling the Parker instability in a rotating plasma screw pinch, Physics of Plasmas 19(2) (2012) 022107-022107-10.

[34] J. Kim, D. Ryu, The effect of the random magnetic field component on the Parker instability, Astrophys. J. 561(1) (2001) L135-L138.

[35] J. Kim, D. Ryu, S.S. Hong, S.M. Lee, J. Franco, The Parker instability, How does the Galaxy Work? Astrophysics and Space Science Library 315 (2005) 315-322.

[36] J. Kim, D. Ryu, T.W. Jones, Three-dimensional simulations of the Parker instability in a uniformlyrotating disk, Astrophys. J. 557(1) (2000) 464-474.

[37] G. Kowal, G. Hanasz, G. Otmianowska-Mazur, Resistive MHD simulations of the Parker instability in galactic disks, Astron. and Astrophys. 315 (2005) 315-322.

[38] M. Kruskal, M. Schwarzschild, Some instabilities of a completely ionized plasma, Proc. Roy. Soc. 
(London) A 233 (1954) 348-360.

[39] T. Kudoh, T. Yokoyama, R. Matsumoto, Magnetohydrodynamic numerical simulation of parker instability in interstellar gas with cosmic ray pressure, in: JPS Conference Proceedings, volume 1, p. 5105 .

[40] A.G. Kulikovskiy, G.A. Lyubimov, Magnetohydrodynamics (1965).

[41] T. Kuwabara, K. Nakamura, C. Ko, Nonlinear Parker instability with the effect of cosmic-ray diffusion, The Astrophysical Journal 607 (2004) 828.

[42] F. H. Lin, L. Xu, P. Zhang, Global small solutions of 2-D incompressible MHD system, J. Differential Equations 259 (2015) 5440-5485.

[43] F. H. Lin, P. Zhang, Global small solutions to an MHD-type system: the three-dimensional case, Comm. Pure Appl. Math. 67 (2014) 531-580.

[44] M. Machida, K.E. Nakamura, T. Kudoh, T. Akahori, Y. Sofue, R. Matsumoto, Dynamo activities driven by magnetorotational instability and the Parker instability in galactic gaseous disks, The Astrophysical Journal 764 (2013) 81.

[45] K.A. Mizerski, C.R. Davies1, D.W. Hughes, Short-wavelength magnetic buoyancy instability, The Astrophysical Journal Supplement Series 205 (2013) 498-518.

[46] F. Morenoinsertis, Nonlinear time-evolution of kink-unstable magnetic flux tubes in the convective zone of the sun, Astron. and Astrophys. 166(1-2) (1986) 291-305.

[47] W. Newcomb, Convective instability induced by gravity in a plasma with a frozen-in magnetic field, The Physics of Fluids 4 (1961) 391-396.

[48] A. Novotnỳ, I. Straškraba, Introduction to the Mathematical Theory of Compressible Flow, Oxford University Press, USA, 2004.

[49] R.H. Pan, Y. Zhou, Y. Zhu, Global classical solutions of three dimensional viscous MHD system without magnetic diffusion on periodic boxes, Archive for Rational Mechanics Analysis 227(2) (2018) 637-662.

[50] E.N. Parker, The formation of sunspots from the solar toroidal field, Astroghys. J. 121 (1955) 491-507.

[51] E.N. Parker, The dynamical state of the interstellar gas and field, Astroghys. J. 145 (1966) 811-833.

[52] J.H. Piddington, An electrodynarnic model of radio galaxies and quasars, Monthly Notices Roy. Astron. Soc. 148 (1970) 131.

[53] X.X. Ren, J.H. Wu, Z.Y. Xiang, Z.F. Zhang, Global existence and decay of smooth solution for the 2-DMHD equations without magnetic diffusion, J. Funct. Anal. 267 (2014) 503-541.

[54] X.X. Ren, Z.Y. Xiang, Z.F. Zhang, Global existence and decay of smooth solutions for the 3-D MHD-type equations without magnetic diffusion, Scientia Sinica Mathematica 59(10) (2016) 1-26.

[55] L.F.S. Rodrigue, G.R. Sarson, A. Shukurov, P.J. Bushby, A. Fletcher, The Parker instability in disk galaxies, Astrophys. J. 816(1) (2015) 691-695.

[56] K. Schwarzschild, On the equilibrium of the sun's atmosphere, Nachr. Kgl. Ges. Wiss. Göttingen (1906) p.41.

[57] K. Shibata, R. Matsumoto, Formation of giant molecular clouds and helical magnetic fields by the Parker instablity, Nature 353 (1991) 633-635.

[58] K. Shibata, T. Tajima, R. Matsumoto, Self-similar evolution of the nonlinear magnetic buoyancy instability, Physics of Fluids B-Plasma Physics 2 (1990) 1989-2000.

[59] Y. Sofue, M. Tosa, Rising and falling motions of gas above the Perseus arm, Astron. and Astrophys. 36 (1974) 237.

[60] Z. Tan, Y.J. Wang, Global well-posedness of an initial-boundary value problem for viscous nonresistive MHD systems, SIAM Journal on Mathematical Analysis 50(1) (2018) 1432-1470.

[61] J.H. Thomas, A.H. Nye, Convective instability in the presence of a nonuniform horizontal magnetic field, Phys. Fluids 18 (1975) 490-491.

[62] M. Tosa, T. Sofue, Rising and falling motion of gas above the spiral arm, Astron. and Astrophys. $32(1974) 461$. 
[63] Y.A. Tserkovnikov, The question of convectional instability of a plasma, Soviet Phys. Dokl. (English transl.) 5 (1960) 87.

[64] G.M. Vasil, N.H. Brummell, Constraints on the magnetic buoyancy instabilities of a shear-generated magnetic layer, Astrophys. J. 686(1) (2008) 709-730.

[65] Y.J. Wang, Critical magnetic number in the MHD Rayleigh-Taylor instability, Journal of Math. Phys. 53 (2012) 073701.

[66] Y.J. Wang, The incompressible viscous non-resistive MHD internal wave problem in a 3D slab, arXiv:1602.02554v1 [math.AP] 8 Feb 2016 (2016).

[67] L. Xu, P. Zhang, Global small solutions to three-dimensional incompressible magnetohydrodynamical system, SIAM J. Math. Anal. 47 (2015) 26-65.

[68] C.P. Yu, Convective stability in a plasma with horizontal magnetic fields, Phys. Fluids 9 (1966) 412-414. 